\title{
On Demand Nanoscale Phase Manipulation of Vanadium Dioxide by Scanning Probe Lithography
}

\author{
Dustin Schrecongost \\ West Virginia University, schrecongost39@gmail.com
}

Follow this and additional works at: https://researchrepository.wvu.edu/etd

Part of the Condensed Matter Physics Commons

\section{Recommended Citation}

Schrecongost, Dustin, "On Demand Nanoscale Phase Manipulation of Vanadium Dioxide by Scanning Probe Lithography" (2020). Graduate Theses, Dissertations, and Problem Reports. 7729.

https://researchrepository.wvu.edu/etd/7729

This Dissertation is protected by copyright and/or related rights. It has been brought to you by the The Research Repository @ WVU with permission from the rights-holder(s). You are free to use this Dissertation in any way that is permitted by the copyright and related rights legislation that applies to your use. For other uses you must obtain permission from the rights-holder(s) directly, unless additional rights are indicated by a Creative Commons license in the record and/ or on the work itself. This Dissertation has been accepted for inclusion in WVU Graduate Theses, Dissertations, and Problem Reports collection by an authorized administrator of The Research Repository @ WVU.

For more information, please contact researchrepository@mail.wvu.edu. 


\title{
On Demand Nanoscale Phase Manipulation of Vanadium Dioxide by Scanning Probe Lithography
}

\author{
Dustin Schrecongost \\ Dissertation submitted \\ to the Eberly College of Arts and Sciences \\ at West Virginia University \\ in partial fulfillment of the requirements for the degree of \\ Doctor of Philosophy in \\ Physics - Condensed Matter \\ Cheng Cen, Ph.D., Chair \\ Lian Li, Ph.D. \\ Aldo Romero, Ph.D. \\ Mikel Holcomb, Ph.D. \\ Matthew Johnson, Ph.D. \\ Nianqiang Wu, Ph.D.
}

Department of Physics and Astronomy

Morgantown, West Virginia

2020

Keywords: Strongly correlated materials, phase change materials, scanning probe lithography, oxygen vacancy, SNOM, KPFM, diffusion, metasurface 


\section{Abstract \\ On-Demand Nanoscale Phase Manipulation of Vanadium Dioxide by Scanning Probe Lithography}

\section{Dustin Schrecongost}

This dissertation focuses on nanoscale phase manipulations of Vanadium Dioxide. Nanoscale control of material properties is a current obstacle for the next generation of optoelectronic and photonic devices. Vanadium Dioxide is a strongly correlated material with an insulator-metal phase transition at approximately $345 \mathrm{~K}$ that generates dramatic electronic and optical property changes. However, the development of industry device application based on this phenomenon has been limited thus far due to the macroscopic scale and the volatile nature of the phase transition. In this work these limitations are assessed and circumvented.

A home-built, variable temperature, scanning near-field optical microscope was engineered for Vanadium Dioxide manipulations and detections. Using this instrument, various scanning probe lithography based methods are implemented to induce new nanoscale phases. Three new phase transitions are discovered; a monoclinic metallic at the nanoscale, a rutile metallic metastable phase, and a van der Waals layered insulator. These new phases are studied and characterized to further understand phase manipulations in strongly correlated materials. One of the new phase transitions, monoclinic metallic, showcases plasmonic excitations. This phenomenon is used to demonstrate various nanoplasmonic devices such as rewritable waveguides, spatially modulated resonators, and reconfigurable planar optics. Finally, Oxygen Vacancy diffusion of the monoclinic structure is monitored to determine the temporal limitation for device applications. The discovery, demonstration, and study of these phases clearly shows the ability to manipulate Vanadium Dioxide on the nanoscale for the first time. Phase control is accomplished under ambient conditions and is stable over long periods of time. This technology opens the door for multifunctional device application using strongly correlated materials. 


\section{Acknowledgements}

This dissertation required more than just one man's effort and dedication. An entire spectrum of Physicists collaborated to make this research possible. All of whom deserve recognition for their contributions.

Simulations and computational insight were provided by Yinxiao Xiang and Aldo Romero.

Sample growths and fabrications were done by Prakash Gajurel, Weitao Dai, Jun Chen, Cuifeng Ying, Amit KC, David Lederman, Hai-Tian Zhang, and Roman Engel-Herbert.

Various experimental contributions came from Mina Aziziha, I-Cheng Tung, Joseph Tessmer, Qiang Wang, Haidan Wen, Yoosuf N. Picard, Ming Yang, Yi Liang, Trent Johnson, and Pavel Borisov.

Of course a more specific thank you goes to my academic advisor, Cheng Cen, for overseeing the entire project from start to finish with me. She provided wisdom, drive, expertise in all facets, and the funding necessary to sustain the project. This thank you is also extended to the DOE and NSF for funding the project. 


\section{Table of Contents}

Acknowledgements............................................................. iii

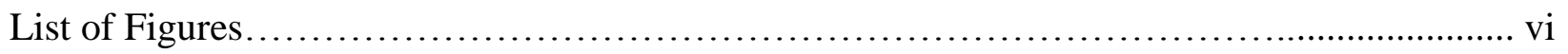

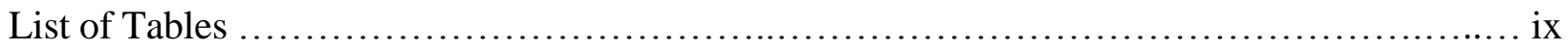

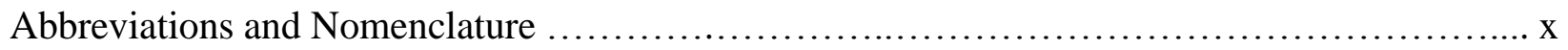

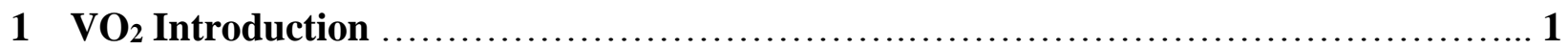

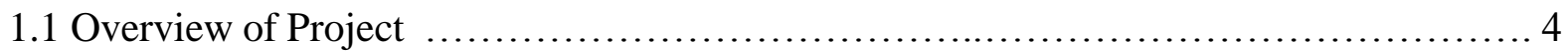

1.2 Properties of Thermal $\mathrm{VO}_{2}$ Insulator to Metal Phase Transition ...................... 5

$1.3 \mathrm{VO}_{2}$ Phase Transitions Activated by Other Stimuli $\ldots \ldots \ldots \ldots \ldots \ldots \ldots \ldots \ldots \ldots \ldots \ldots . \ldots$

2 Scanning Probe Imaging Modes \& Designing a Home-built SNOM................... 12

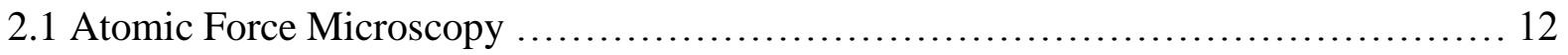

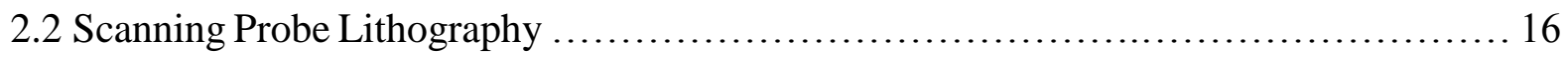

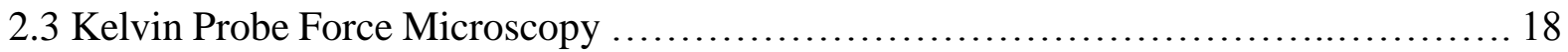

2.4 Scanning Near-field Optical Microscopy \& Instrument Design ...................... 21

2.5 In Situ KPFM + SNOM .................................................. 32

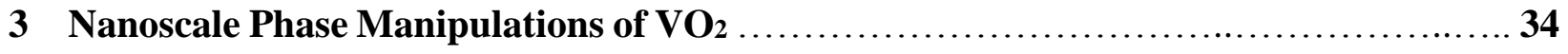

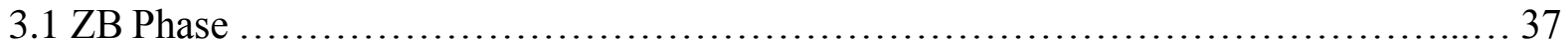

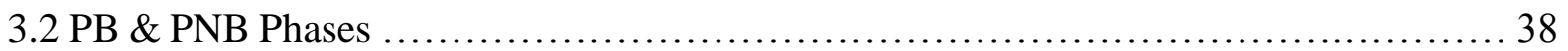

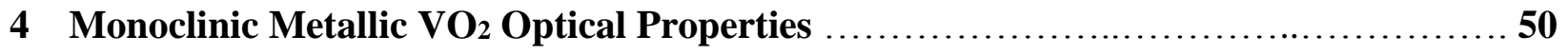

4.1 Optical \& Plasmonic Properties ............................................ 50

4.2 Demonstration of $\mathrm{VO}_{2}$ Nanophotonic Devices ................................ 57

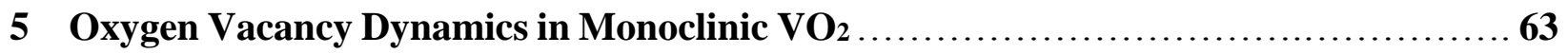

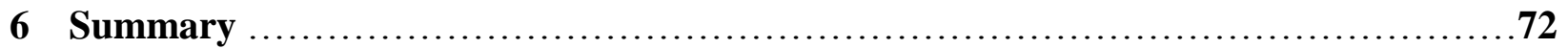


6.1 Outlook

Appendix A Ferroelectric Domain Orientation in Multiferroic Thin Film BaCoF 4........76

Appendix B Reconfigurable Modulation of LAO/STO Photovoltage Mechanism.........83

References 


\section{List of Figures}

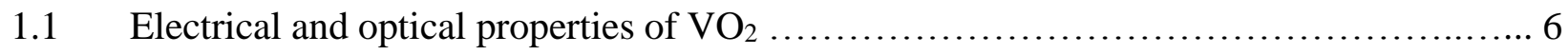

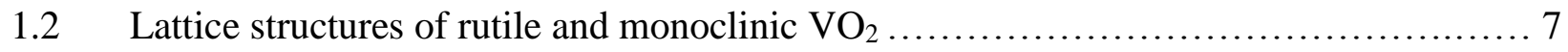

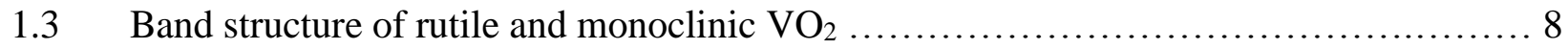

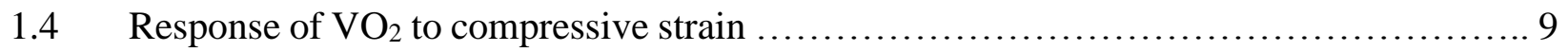

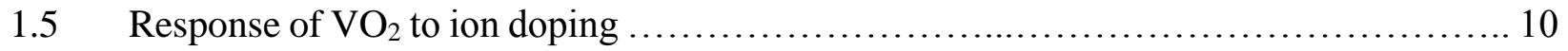

$2.1 \quad$ Illustration of AFM cantilever ................................................... 13

2.2 AFM force vs distance approach curve .......................................... 13

2.3 Illustration \& example of AFM measurement ..................................... 14

2.4 Diagram of various scanning probe lithography methods ......................... 16

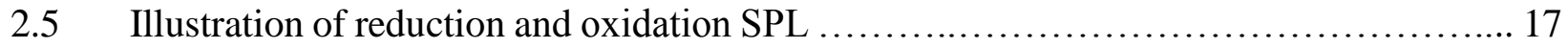

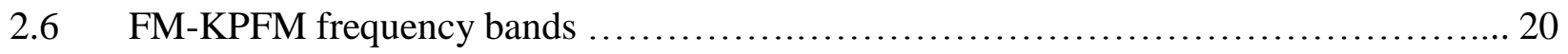

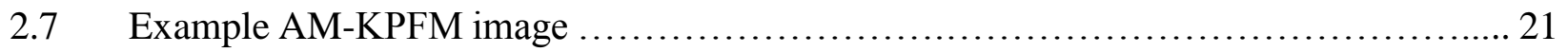

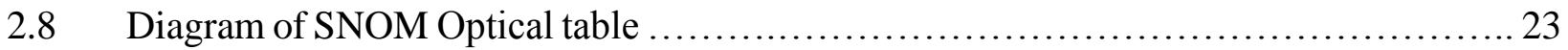

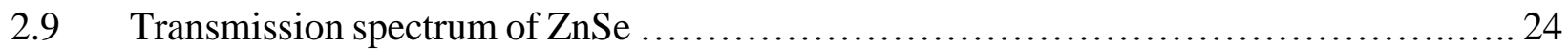

2.10 SNOM images of high harmonic background suppression ........................... 25

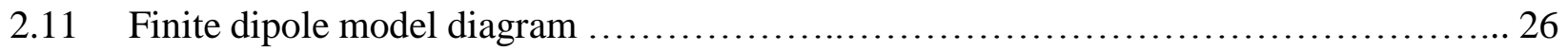

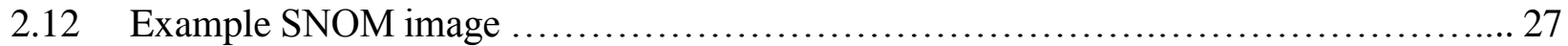

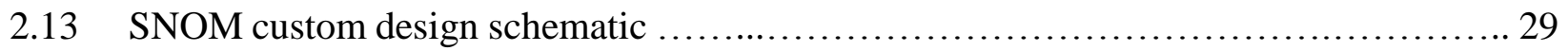

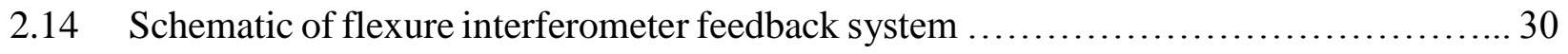

2.15 Flexure interferometer displacement measurement ….............................. 31

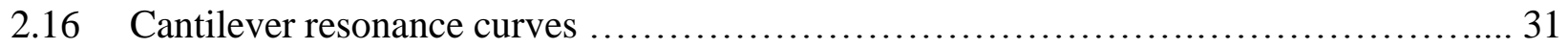

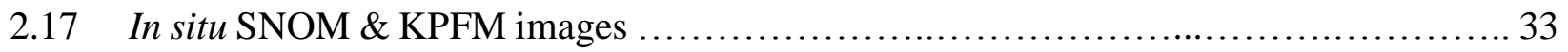

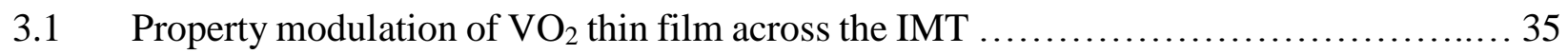


3.2 Overview of scanning probe induced phase transitions .............................. 36

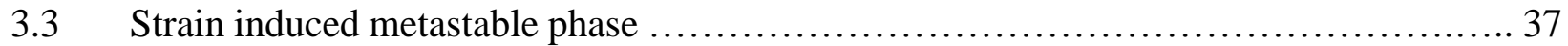

3.4 Surface topography and electrical properties of PB and PNB phases .................... 39

3.5 XPS spectrums measured in different $\mathrm{VO}_{2}$ phases ................................ 40

3.6 Images of PNB structures with different writing speeds ............................ 41

3.7 Structural properties by $\mu$ XRD spectrums of phases near the monoclinic (200) peak .... 42

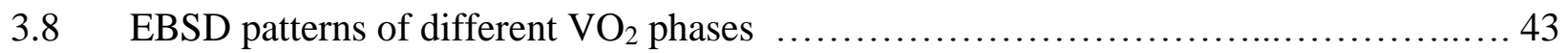

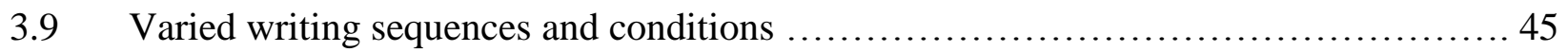

3.10 Hydrophilic water contact angle image ........................................... 46

3.11 Raman spectra of different $\mathrm{VO}_{2}$ phases ........................................ 48

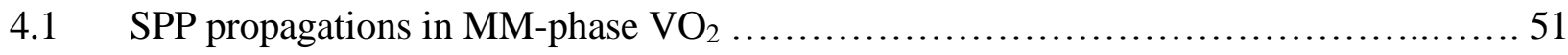

4.2 Maps of the complex permittivities matching observed SPP mode .................... 54

4.3 Relative reflectance comparing metallic $\mathrm{VO}_{2}$ phases …............................. 55

$4.4 \quad$ Film thickness dependence of SPP modes ...................................... 56

4.5 Spatially modulated plasmonic resonances ..................................... 58

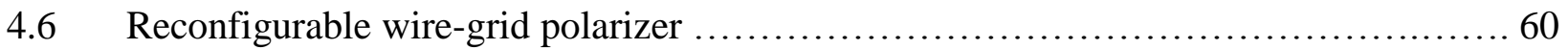

4.7 Size-dependent optical performance of $\mathrm{MM}-\mathrm{VO}_{2}$ structures $\ldots \ldots \ldots \ldots \ldots \ldots \ldots \ldots \ldots \ldots \ldots$

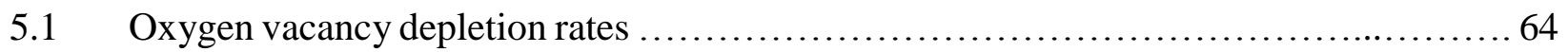

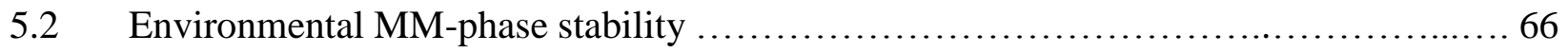

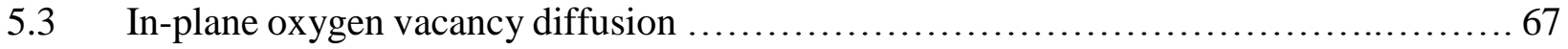

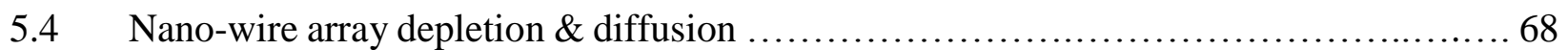

5.5 Monoclinic vs rutile metallic domain dynamics ................................... 69

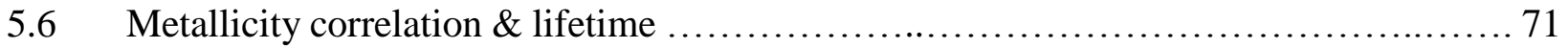

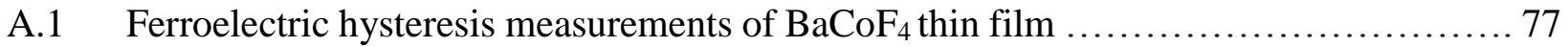


A.2 PFM results for domain orientations of $\mathrm{BaCoF}_{4}$ thin film......................... 79

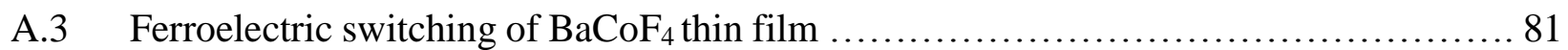

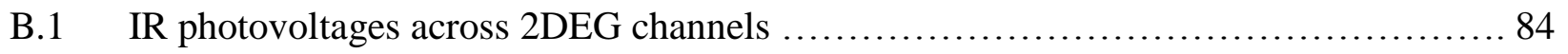

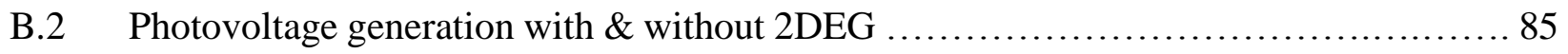

B.3 Temperature dependences of the photovoltage at LAO/STO interfaces .............. 87

\section{List of Tables}

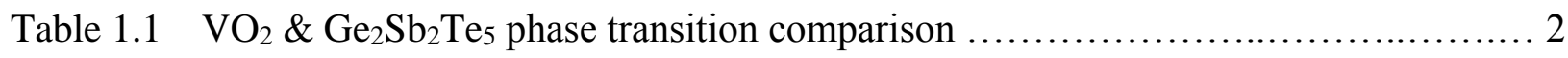

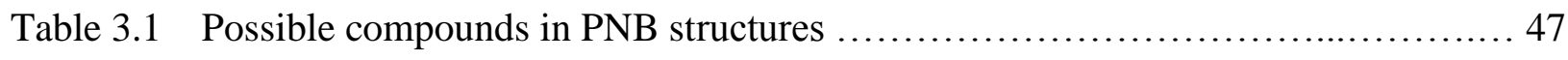

Table 4.1 MIR $(10.9 \mu \mathrm{m})$ complex permittivities of VO2 phases $\ldots \ldots \ldots \ldots \ldots \ldots \ldots \ldots \ldots \ldots 55$ 


\section{Abbreviations and Nomenclature}

2DEG: 2D Electron Gas

AFM: Atomic Force Microscope

AM-KPFM: Amplitude Modulation Kelvin Probe Force Microscopy

EBSD: Electron Back Scatter Diffraction

FDTD: Finite Difference Time Domain

FEM: Finite Element Method

FM-KPFM: Frequency Modulation Kelvin Probe Force Microscopy

FWHM: Full Width at Half Maximum

IMT: Insulator-Metal Transition

KPFM: Kelvin Probe Force Microscopy

MBE: Molecular Beam Epitaxy

MCT: HgCdTe

MI: Monoclinic Insulator

MIR: Mid Infrared

MM: Monoclinic Metal

$\mu$ XRD: Micro X-Ray Diffraction

NB: Negative Bias

NIR: Near Infrared

NPB: Negative Bias Followed by Positive Bias

OV: Oxygen Vacancy

PB: Positive Bias

PFM: Piezoresponse Force Microscopy

PNB: Positive Bias Followed by Negative Bias 
PTE: Photothermoelectric

Ro: Current Limiting Resistor

Rc: Contact Resistance

RM: Rutile Metal

RT: Room Temperature

SPL: Scanning Probe Lithography

SPP: Surface Plasmon Polariton

SNOM: Scanning Near-field Optical Microscopy

Tc: Critical Temperature

uc: unit cell

UV: Ultra-Violet

VO2: Vanadium Dioxide

XPS: X-ray Photoelectron Spectroscopy

XRD: X-ray Diffraction

ZB: Zero Bias 


\section{Chapter 1}

\section{$\mathrm{VO}_{2}$ Introduction}

Optoelectronic and photonic devices are part of our way of living in the year 2020. The general population is ignorant to the significance this technological field has on their everyday lives. These devices are the backbone of high speed communications, information processing, entertainment devices, and medical instrumentation ${ }^{1-3}$. Photonic devices rely on our ability to control and manipulate light waves through their amplitude, phase, and polarization ${ }^{4}$. Conventional refractive optics require a long propagation distance to accumulate substantial effects due to the refractive index of the optical element. However, this necessary spatial footprint is not compatible with progressive technology miniaturization ${ }^{5}$. This spatial limitation leads into the field of artificial media for manipulating light waves.

The idea to engineer artificial materials has been around since the end of the $19^{\text {th }}$ century . Metamaterials are artificially structured materials that exhibit extraordinary electromagnetic properties not available in nature ${ }^{7}$. They have been used to demonstrate phenomenon and performance such as negative index of refraction ${ }^{8-10}$, perfect lenses ${ }^{11,12}$, perfect absorbers ${ }^{13-15}$, electromagnetic chirality ${ }^{16}$, and enhanced nonlinear effects ${ }^{17,18}$. An extension of the 3D metamaterial is the 2D version, a metasurface. Metasurfaces are based on Huygens' principle; every point on a wavefront is itself the source of a spherical wavelet that all mutually interfere to result in the overall wavefront ${ }^{19,20}$. Subwavelength arrays of planar structures are patterned to generate surface currents which effect individual pixels of the total wavefront ${ }^{20}$. This subwavelength manipulation over the entire wave allows for unique optical engineering, and the planar nature of metasurfaces makes them on-chip compatible for nanophotonic devices in future technology ${ }^{21}$.

A current trend in metadevices, metasurface based devices, is to incorporate dynamic tunability or modulation. If the metasurface arrays can be distorted, the overall function of the metadevice can be manipulated ${ }^{22}$. For example if the length of a rectangular, metal slab is affected, the resonant wavelength for constructive interference has been adjusted. The idea is identical to standing waves on a string, interference conditions are a function of length. This array distortion 
has been accomplished using microelectromechanical actuators to vary spatial parameters of the metasurface $^{23}$. Although, an alternative, more passive option for metadevice tuning and modulation is to add an active layer to the device. This is a separate heterostructure layer that can be manipulated by a variety of stimuli to alter electromagnetic properties. The goal is to alter the electromagnetic environment around the metasurface arrays and thus the interaction between array elements $^{24,25}$. Liquid crystals ${ }^{26,27}$, ferroelectrics ${ }^{28}$, graphene ${ }^{29-31}$, strongly correlated materials ${ }^{32-34}$, and chalcogenide glasses ${ }^{35-37}$ have all successfully been used as active layers to create multifunctional metadevices. However, two material families are primarily used as these active layers because of their drastic and reliable electromagnetic property tunability; Vanadium Oxides and chalcogenide phase change glasses. The specific compounds used are $\mathrm{VO}_{2}$ and GST; $\mathrm{Ge}_{2} \mathrm{Sb}_{2} \mathrm{Te}_{5}$ or $\mathrm{Ge}_{3} \mathrm{Sb}_{2} \mathrm{Te}_{6}$ depending on specifically desired optical properties ${ }^{38-40}$.

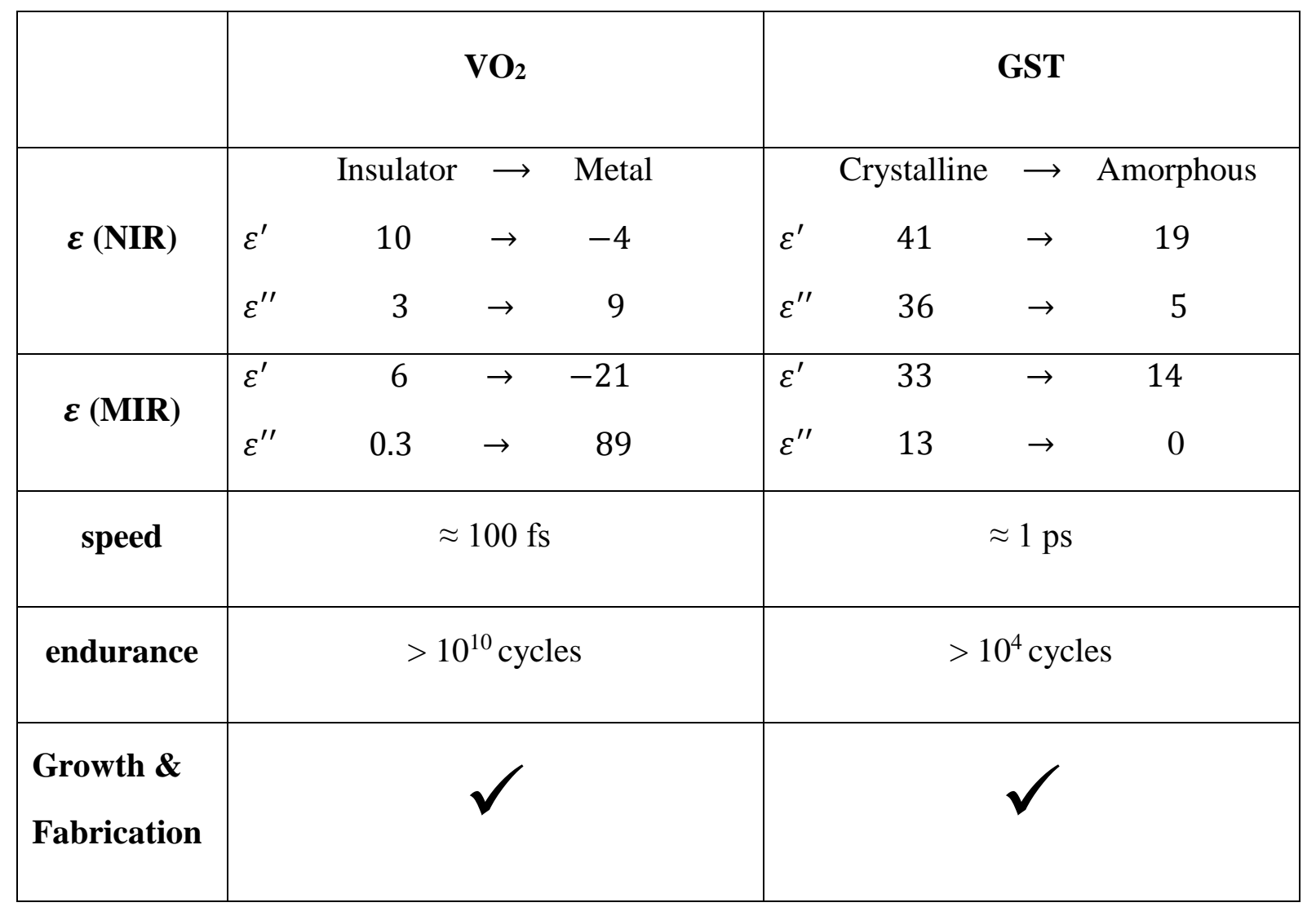

Table $1.1 \mathrm{VO}_{2} \& \mathrm{Ge}_{2} \mathrm{Sb}_{2} \mathrm{Te}_{5}$ phase transition comparison. Both $\mathrm{VO}_{2}$ and $\mathrm{Ge}_{2} \mathrm{Sb}_{2} \mathrm{Te}_{5}$ have drastic optical property changes across their phase transition in the NIR and MIR. Both happen on ultrafast time scales, have high endurance, and are mature in their growth/fabrication technology. 
Both of these materials are considered phase change materials where a phase transition leads to the electromagnetic property variation. As can be seen in Table 1.1, both materials have drastic optical property changes across their phase transition in the near infrared (NIR) ${ }^{41-43}$ and mid infrared (MIR) $)^{43-45}$, both happen on ultrafast time scales ${ }^{46,47}$, have high endurance ${ }^{48,49}$, and are mature in their fabrication/growth technology ${ }^{50-56}$. I will note that Table 1.1 is an overview of material properties that will vary based on growth conditions and phase transition activation mechanism, but it summarizes the overall appeal of these materials. These materials seem similar, but the phase transitions are very different. GST is most commonly in a semiconducting, amorphous state with minimal free charge carriers so a lack of optical absorption. Upon annealing GST, it crystallizes with resonant bonds that induce a free carrier density leading to the modulation of optical properties. Further melting and quickly quenching the GST will reset the phase back to the amorphous state ${ }^{43,57,58}$. In contrast, $\mathrm{VO}_{2}$ is a first order phase transition where it changes from an insulator to a metal due to electron correlation effects. The lattice remains crystalline across the phase transition albeit with a modified lattice structure. The $\mathrm{VO}_{2}$ phase transition will be further discussed in sections 1.2 and 1.3 as this material is the focus of this work.

GST is exclusively used for industrial applications such as optical data storage like rewritable DVDs, CDs, and blue rays ${ }^{38,59-61}$. Thus the question becomes why is GST chosen over $\mathrm{VO}_{2}$ in industry? There are three specific drawbacks in $\mathrm{VO}_{2}$ technology that has kept it less relevant for industrial applications. This dissertation will provide knowledge and solutions to these issues through "On Demand Nanoscale Phase Manipulation of Vanadium Dioxide by Scanning Probe Lithography".

1. The $\mathrm{VO}_{2}$ insulator-metal phase transition (IMT) is most commonly achieved through thermal activation. Thus the need to heat the material automatically means that the IMT cannot be controlled under ambient conditions. There has been progress in activating the phase transition with other stimuli such as doping and strain which will be discussed in section 1.3. However, the ability to activate and reset the IMT in an on-demand manner under ambient conditions has remained elusive.

2. The $\mathrm{VO}_{2} \mathrm{IMT}$ is a volatile phase transition. The $\mathrm{VO}_{2}$ is heated above the critical transition temperature $\left(\mathrm{T}_{\mathrm{c}}\right)$ to activate the IMT, and then the temperature has to remain elevated or the $\mathrm{VO}_{2}$ will transition back to the insulating phase upon cooling. The need to maintain the 
metallic phase temperature is not energy efficient for application. Doping the $\mathrm{VO}_{2}$ with Oxygen vacancies (OVs) has recently shown a more stable phase transition ${ }^{62,63}$, but further understanding of the $\mathrm{OV}$ dynamics is needed before proclaiming a truly non-volatile $\mathrm{VO}_{2}$ phase transition.

3. $\mathrm{VO}_{2}$ phase transitions are on a macroscopic scale. The entire film undergoes the IMT. This works for overall modulation, but limits any spatial variation for a high density of binary data storage. Recently it has been demonstrated that microscale phase transitions can be accomplished through defect engineering ${ }^{64}$, but a volatile heating process is still necessary to control phase transitions at multiple critical temperatures. Local domain control in ambient conditions and in a non-volatile manner is desired for reconfigurable spatial control over device functionality or monolithic photonic devices. Also achieving nanoscale spatial resolution would make the phase transition on-chip compatible with current telecom nanophotonics 65 .

\subsection{Overview of Project}

Going forward, the remainder of chapter 1 will be dedicated to background information on the $\mathrm{VO}_{2}$ IMT. The optical and electrical property variation of both $\mathrm{VO}_{2}$ phases (insulating and metallic) will be compared. Then the corresponding structural transition will be analyzed along with the band structure of each phase. Lastly, a variety of phase transition stimuli will be discussed. Typically the phase change is activated thermally but doping, strain, field effect, and UV light have also been proven reliable.

Chapter 2 will be dedicated to instrumentation for the experimental work performed in this dissertation. The bulk of the experiments were performed using a home-built Scanning Near-field Optical Microscope (SNOM). This instrument was engineered specifically for SNOM, and is also capable of other common scanning probe based imaging modes along with common reflectance optical measurements. Chapter 2 will discuss the various imaging modes performed with this home-built instrument along with unique design features.

Chapter 3 will study on demand, nanoscale phase manipulations of $\mathrm{VO}_{2}$ under ambient conditions. Three new IMT activation methods are demonstrated and their base electromagnetic properties characterized. The most applicable of these phases is then chosen for further analysis in 
chapter 4. The MIR optical and plasmonic properties are diagnosed in detail and utilized to demonstrate nanoplasmonic devices. Then in chapter 5 metallic domain lifetimes are monitored to understand OV dynamics. The acquired data can then be used to model future device lifetimes.

Finally this work is concluded along with an outlook on how this research can benefit the scientific community. The local optical property variation may benefit society's transition towards integrated photonic circuitry. Also the plasmonic properties of metallic $\mathrm{VO}_{2}$ will allow for a monolithic optical engineering platform. Appendices A, B are attached at the end to shed light on other examples of scanning probe based nanoscale manipulations and measurements. Multiferroic domains are able to be identified, and their electric dipole orientation analyzed and even reorientated in some cases. Also photovoltaic modulation of complex oxides is demonstrated by creating and erasing local electron gases. Scanning probe based manipulations are opening up new opportunities in a variety of solid-state physics research areas.

\subsection{Properties of Thermal $\mathrm{VO}_{2}$ Insulator to Metal Phase Transition}

$\mathrm{VO}_{2}$ is most well-known for its first order IMT. At room temperature it is an insulator, but at approximately $345 \mathrm{~K}$ metallic puddles begin to form and percolate as it undergoes a phase transition to a metallic state due to its strongly correlated nature of $\mathrm{d}$ band electrons ${ }^{66,67}$. More specifically, it is a Mott insulator; a class of materials where Coulomb repulsion between strongly correlated electrons dominates over all other processes and blocks electron motion at low temperatures and energies ${ }^{68}$. The coulomb repulsion introduces an energy barrier for the electrons to hop between lattice sites, but at elevated temperatures the material overcomes this energy barrier and becomes metallic. Several Vanadium Oxide compounds undergo this IMT. However, the transition temperature in $\mathrm{VO}_{2}(345 \mathrm{~K})$ is easily obtainable, and thus has made $\mathrm{VO}_{2}$ an attractive candidate for studying strongly correlated materials ${ }^{69}$. The $\mathrm{VO}_{2}$ IMT causes drastic electric $66,70,71$ and optical ${ }^{41,42,44,45}$ property changes as can be seen in figure 1.1. The resistivity decreases approximately 3-4 orders of magnitude in thin films, and up to 5 orders in bulk form ${ }^{72}$. Optically, the room temperature phase is practically transparent $(\mathrm{k} \sim 0)$ and metallic at high temperatures $(\mathrm{n}$ $<\mathrm{k}$ ) for wavelengths greater than about $1 \mu \mathrm{m}$. The increase in free carrier density increases the absorption of the film in the infrared regime. 

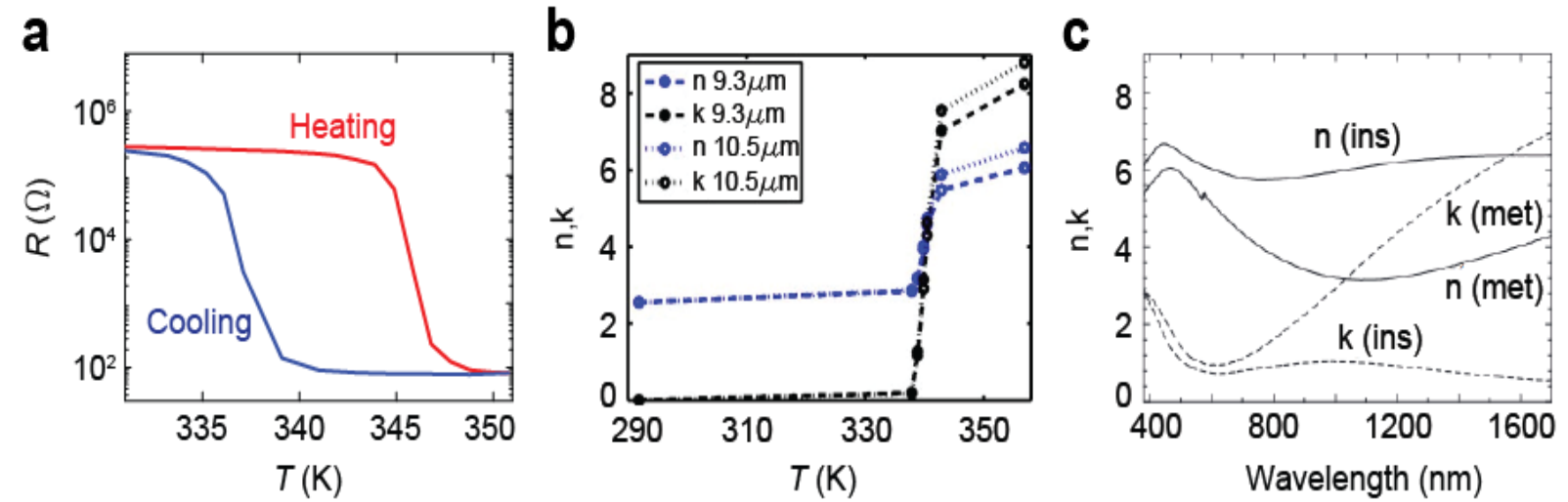

Figure 1.1 Electrical and optical properties of $\mathrm{VO}_{2}$ (a) Resistance vs temperature across the IMT. (b) MIR refractive index across the IMT ${ }^{45}$. (c) Refractive index vs wavelength in the visible and NIR for the metallic and insulating phases ${ }^{41}$.

Simultaneously to these electromagnetic property changes, $\mathrm{VO}_{2}$ undergoes a structural phase transition as well. At high temperatures, it has a rutile lattice with parameters $a_{R}=b_{R}=$ $4.56 \AA, c_{R}=2.85 \AA^{73-75}$. A rutile structure is a body-centered tetragonal lattice. Vanadium atoms are encompassed by 6 Oxygen atoms creating a $\mathrm{VO}_{6}$ octahedron. These octahedron have short VV chains $(2.85 \AA)$ along the $[001]_{R} c_{R}$ axis which is important to the metallic conduction as will be discussed with the metallic phase band structure. Along the other axes, the octahedrons are rotated with respect to each other and no $\mathrm{V}-\mathrm{V}$ chains are formed. A representation of the rutile $\mathrm{VO}_{2}$ lattice can be seen in figure 1.2a. Decreasing the temperature, the $\mathrm{VO}_{2}$ structural phase transition takes place at $T_{c}$. The structure distorts into a monoclinic lattice. The Vanadium atoms displace along the $[110]_{R} \&[1 \overline{1} 0]_{R}$ axes, but are still enclosed within their $\mathrm{VO}_{6}$ octahedra. The monoclinic lattice is shown in figure $1.2 \mathrm{~b}$. The distortion allows for the generation of $\mathrm{V}^{4+}-\mathrm{V}^{4+}$ dimers along the $c_{R}$ axis. The dimers have a short intradimer distance of $2.62 \AA$, and a longer interdimer distance $3.16 \AA^{76}$. This variable distance along the $c_{R}$ axis leads to the insulating characteristics of the monoclinic phase. The monoclinic lattice has parameters $a_{M}=5.75 \AA, b_{M}=$ $4.53 \AA, c_{M}=5.38 \AA, \beta=122.6^{\circ} 77$. The structural phases across the transition can be related to each other via $\vec{a}_{m} \sim-2 \vec{c}_{R}, \vec{b}_{m} \sim-\vec{a}_{R}, \vec{c}_{m} \sim \vec{b}_{R}+\vec{c}_{R}{ }^{78}$. 
a

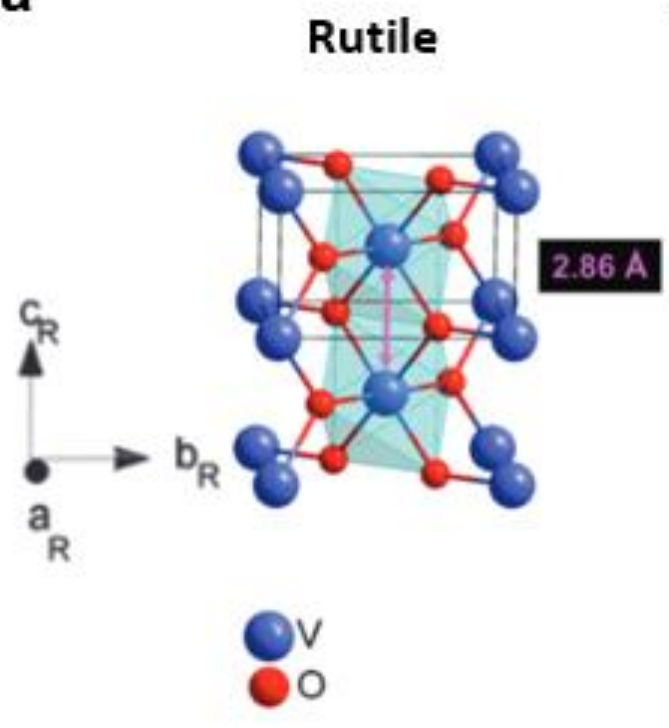

b

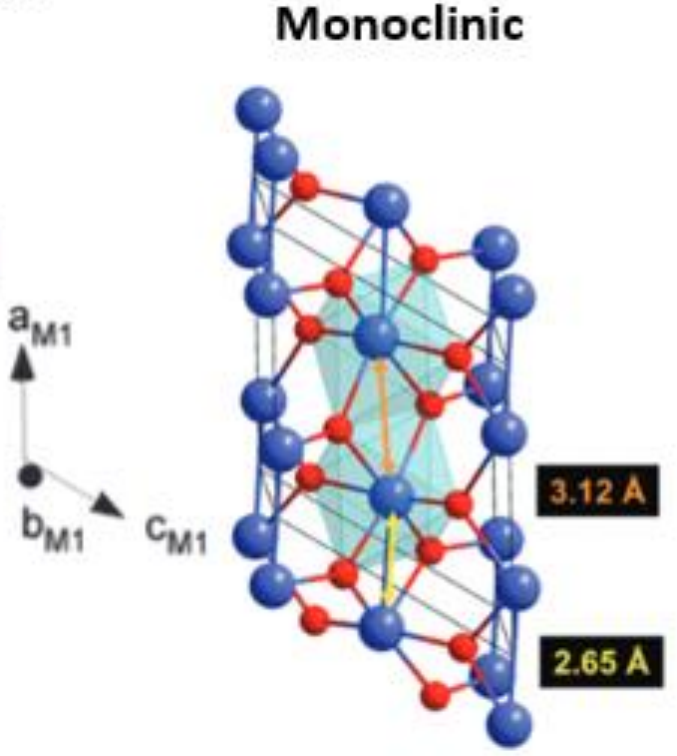

Figure 1.2 Lattice structures of rutile and monoclinic $\mathrm{VO}_{2}($ a) Lattice structure of rutile metallic phase. (b) Lattice structure of monoclinic insulating phase. ${ }^{79}$

The band structure of $\mathrm{VO}_{2}$ near the Fermi level has thoroughly been investigated and is dominated by hybridization of the V $3 \mathrm{~d}$ and $\mathrm{O} 2 \mathrm{p}$ orbitals ${ }^{80-82}$. There are five V $3 \mathrm{~d}$ states available for hybridization; $d_{x y}, d_{x z}, d_{y z}, d_{x^{2}-y^{2}}, d_{z^{2}}$. Two of these orbitals $\left(d_{x y}, d_{z^{2}}\right)$ point directly towards Oxygen atoms forming higher energy $\sigma$ bonds. Another two orbitals $\left(d_{x z}, d_{y z}\right)$ are oriented between the Oxygen atoms forming lower energy $\pi$ bonds. The hybridization between the V $3 \mathrm{~d}$ and $\mathrm{O} 2 \mathrm{p}$ orbitals cause both the $\sigma$ and $\pi$ bonds to split into filled bonding states with $\mathrm{O} 2 \mathrm{p}$ characteristics and anti-bonding states, $\sigma^{*}$ and $\pi^{*}$, with $\mathrm{V} 3 \mathrm{~d}$ characteristics. The $\pi^{*}$ band is partially filled while the higher energy $\sigma^{*}$ band is completely above the Fermi level. The remaining $\mathrm{V} 3 \mathrm{~d}$ state $\left(d_{x^{2}-y^{2}}\right.$ or $\left.d_{/ /}\right)$is also a $\mathrm{V}-\mathrm{O} \pi$ bond but is oriented along the asymmetric $[001]_{R} c_{R}$ axis in the direction of the $\mathrm{V}-\mathrm{V}$ chains. The asymmetry causes a decrease in the $\mathrm{V}-\mathrm{O}$ hybridization, but an increase in $\mathrm{V}-\mathrm{V}$ orbital overlap. This band is a partially filled conducting band. When distorting to the monoclinic lattice, the V-V chain is broken into V-V dimers splitting the $d_{/ /}$band into filled and empty states. Also the V $3 \mathrm{~d}-\mathrm{O} 2 \mathrm{p}$ orbital overlap is increased thus increasing the splitting of bonding and antibonding states. The increased band splitting shifts the $\pi^{*}$ state above the Fermi level. The net result is a band gap of approximately $0.65 \mathrm{eV}^{80-82}$. The band diagrams of the rutile and monoclinic states are shown in figure 1.3. 
a

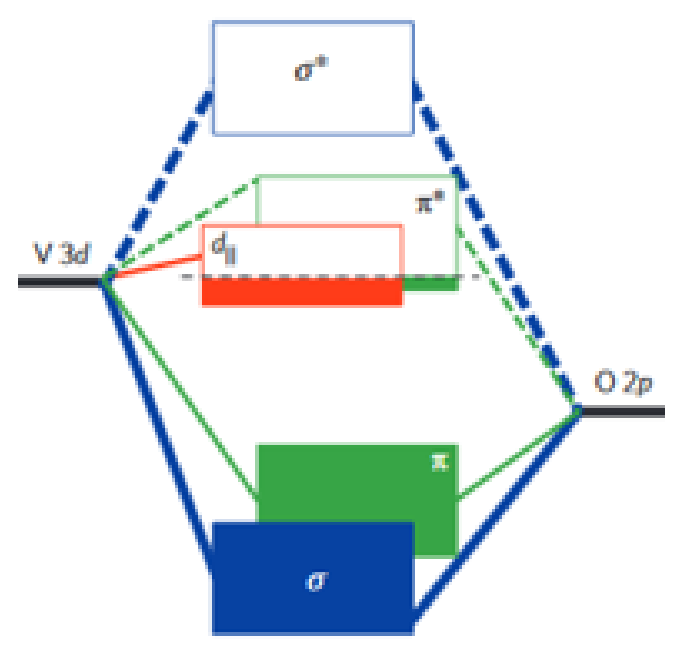

b

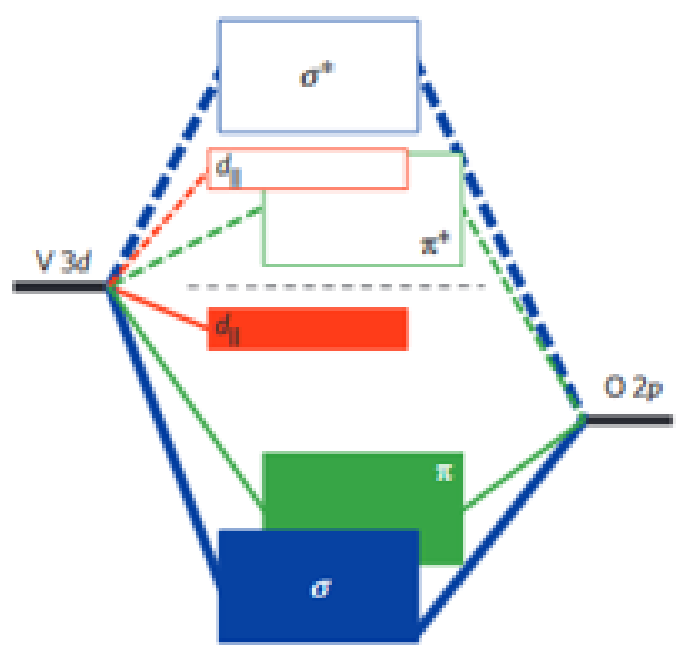

Figure 1.3 Band structure of rutile and monoclinic $\mathrm{VO}_{2}($ a) Rutile metallic phase band structure near Fermi level (b) Monoclinic insulating phase band structure near Fermi level. ${ }^{81}$

One question left about the IMT is which came first the chicken or the egg? Which portion of the phase transition, the IMT or the structural change, is the cause and which is the effect. It has long been debated if $\mathrm{VO}_{2}$ is a Peierls or Mott insulator ${ }^{44,67,78,80,83}$. In the case of the Peierls insulator, the structural transition causes the band splitting and the IMT is the result. This would make $\mathrm{VO}_{2}$ a common band insulator. For a Mott insulator, electron-electron correlations (Coulomb repulsion) are the cause for the band gap. The current consensus is that $\mathrm{VO}_{2}$ is a Mott insulator. Femtosecond pump-probe measurements were performed in situ with temperature dependent $\mathrm{x}$ ray diffraction (XRD) to show the IMT happens first and followed by the structural phase transition $^{84,85}$.

\section{$1.3 \mathrm{VO}_{2}$ Phase Transitions Activated by Other Stimuli}

In recent years, researchers have realized that the strongly correlated nature of $\mathrm{VO}_{2}$ allows for phase transitions to be activated by a variety of stimuli such as strain ${ }^{81,86,87}$, doping ${ }^{88-97}$, field effect gating ${ }^{62,63,82,87,98,99}$, and UV light ${ }^{100}$. These various methods are leading to breakthroughs for local, ambient control over metallic $\mathrm{VO}_{2}$. This section will focus on explaining how and why each method is capable of activating $\mathrm{VO}_{2}$ phase transitions. Variations of these stimuli are used to accomplish on demand nanoscale phase manipulations in this thesis. 
Uniaxial strain applied to $\mathrm{VO}_{2}$ thin films has been shown to greatly affect the IMT temperature ${ }^{62,81,86,87}$ whereas hydrostatic pressure shows minimal effect ${ }^{101}$. Figure 1.4a shows electrical resistivity transition curves for films grown on different substrates ${ }^{62}$. The different substrates provide different strains applied to the films due to lattice mismatch ${ }^{62}$. The $T_{c}$ value is shown to vary by as much as $50 \mathrm{~K}$. The change in $\mathrm{T}_{\mathrm{c}}$ can be understood by thinking about the role that the strain plays with respect to the lattice. First a uniaxial stress (force per area) is applied to a film. Second, in response to this stress the lattice parameters are altered. This change in length is strain; $\eta=\Delta l / l$. The amount of lattice displacement is based on the materials Young's Modulus; $s=E \eta$ where $\mathrm{s}$ is stress and $\mathrm{E}$ is Young's Modulus ${ }^{102} \cdot \mathrm{VO}_{2}$ is most commonly grown with the $c_{R}$ axis oriented out of plane and is the case for the samples used in this thesis. In this scenario, a compressive strain will compress the $c_{R}$ axis which increases the $\mathrm{V}-\mathrm{V}$ orbital overlap of the $d_{/ /}$ band. The result of this will be further splitting of the $d_{/ /}$band in the monoclinic phase. However in contrast, the compressive strain will increase the length of the $a_{R}$ lattice parameter which decreases the V-O orbital overlap. This decreases the splitting of the $\pi$ band, and lowers the $\pi^{*}$ band towards the Fermi level as can be seen in figure 1.4b. The smaller band gap allows for conducting electrons at lower temperatures. This is how strain is used to lower $\mathrm{T}_{\mathrm{c}}$.
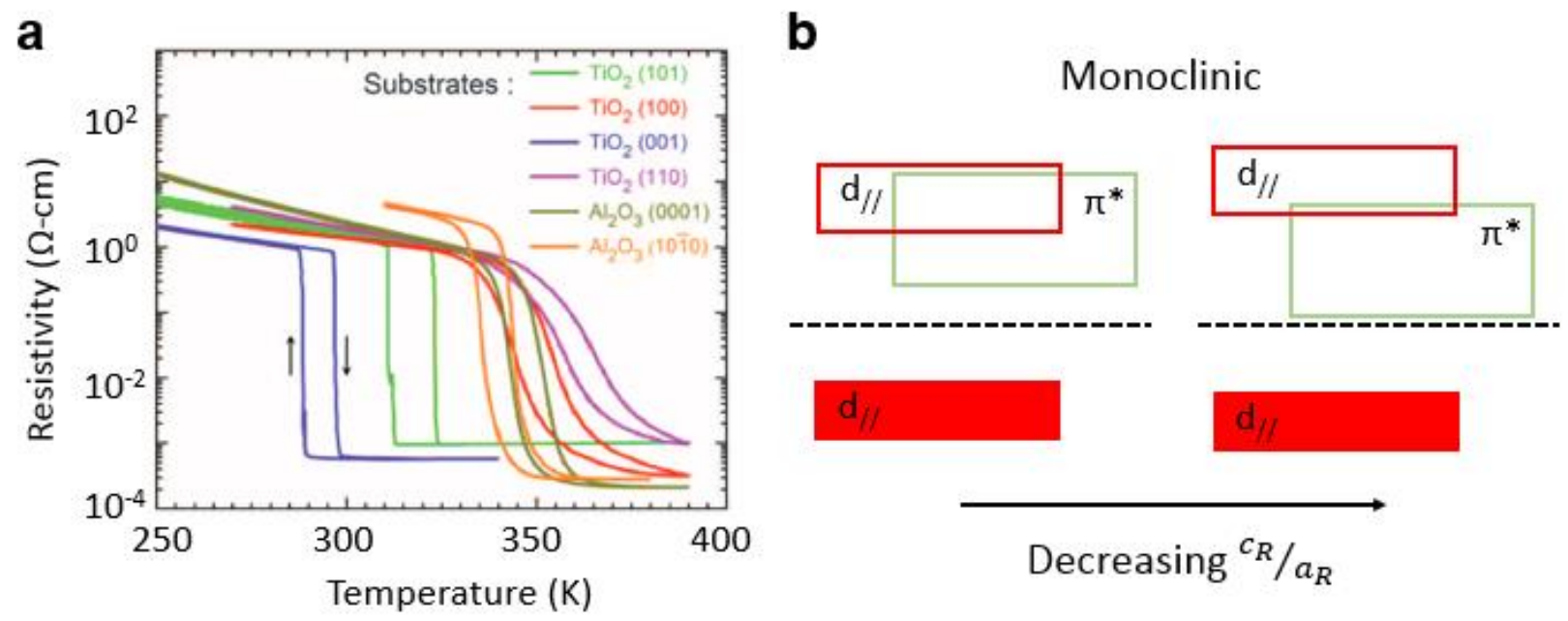

Figure 1.4 Response of $\mathrm{VO}_{2}$ to compressive strain (a) Resistivity vs temperature of $\mathrm{VO}_{2}$ films grown on different substrates to apply different compressive strains along the $c_{R}$ axis ${ }^{62}$ (b) Response of the band structure in the monoclinic phase near the Fermi energy as compressive strain is applied to the out of plane $c_{R}$ axis. 
A second, well established method for shifting the $\mathrm{VO}_{2} \mathrm{~T}_{\mathrm{c}}$ is doping the film ${ }^{88-97}$. A percentage of $\mathrm{V}^{4+}$ ions can be replaced by impurity ions at growth, shifting the charge carrier density and the Fermi energy. Similar to semiconductor band behavior ${ }^{103}$, lower valence impurities can be used to add acceptor states which decrease the Fermi energy. This has been found to increase the $\mathrm{VO}_{2}$ film $\mathrm{T}_{\mathrm{c}}$. Higher valence impurities add donor states which decreases $\mathrm{T}_{\mathrm{c}}$. The general trend of $\mathrm{T}_{\mathrm{c}}$ for different valence doping impurities can be seen in figure 1.5a. Interestingly, doping the $\mathrm{VO}_{2}$ film with other 4+ valence ions shows less effect than other valences ${ }^{91,92}$. However, an effect can still be seen due to the strain applied by the varying ion radius. Thus the larger effect is the change in charge carrier density rather than strain.

a

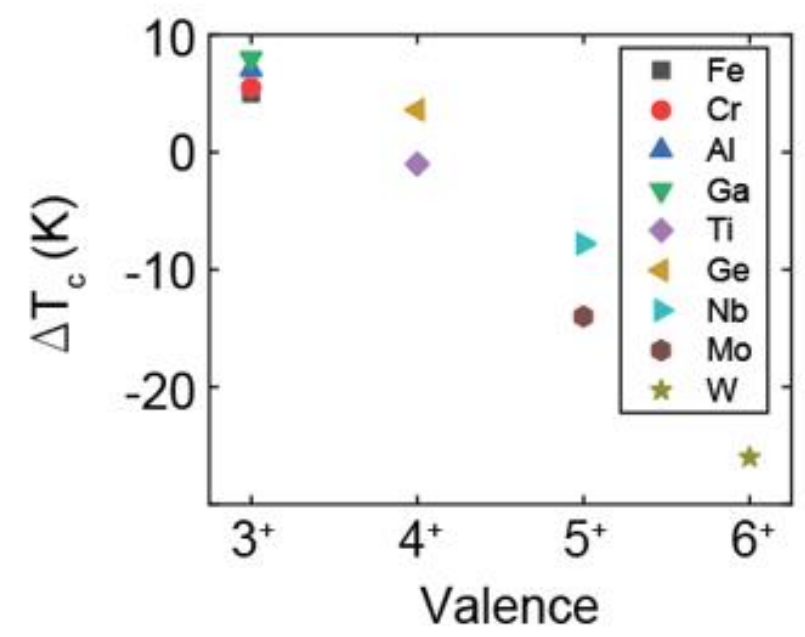

C

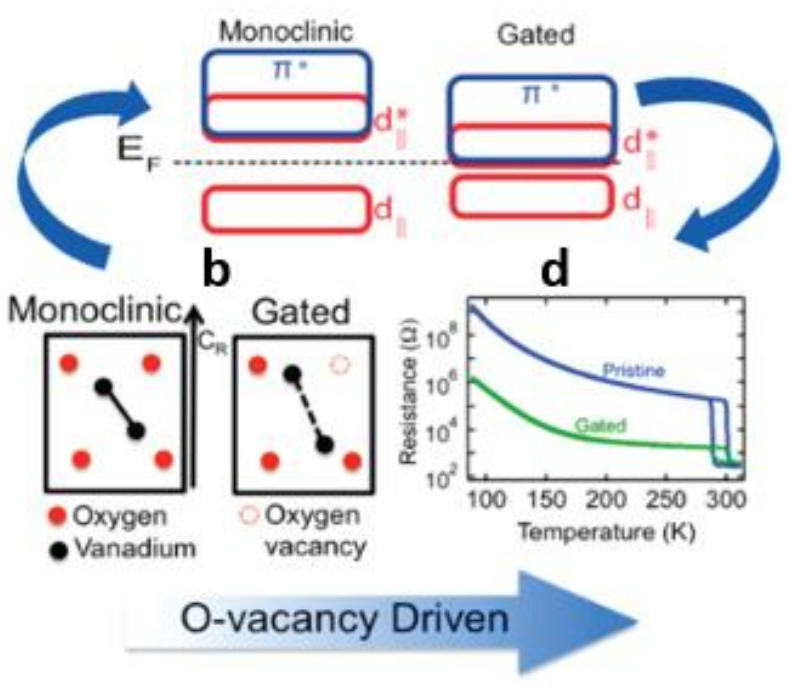

Figure 1.5 Response of $\mathrm{VO}_{2}$ to ion doping (a) The change in $T_{c}$ for different valence ions doped into $\mathrm{VO}_{2}$ films $(\approx 1 \%)$. (b) Lattice structural changes induced by electrolyte gating. (c) Evolution of the band structure due to electrolyte gating. (d) Temperature dependent resistance of pristine $\mathrm{VO}_{2}$ vs gated $\mathrm{VO}_{2}{ }^{99}$

In this thesis, the primary phase transition induced is similar to $\mathrm{VO}_{2}$ phase transitions demonstrated by field effect gating. This stimuli enables defect doping of the $\mathrm{VO}_{2}$ film. Initial experiments were conducted by placing an electrolyte gel on a $\mathrm{VO}_{2}$ film surface. When applying a voltage between the gel and surface with the gel acting as the positive, Oxygen Vacancies (OVs) can be accumulated in the $\mathrm{VO}_{2}$ lattice $62,63,82,99$. The electric field drives a migration of negative Oxygen ions from the film to the electrolyte gel. The gel acts as an electrolyte sink, and the Oxygen 
migration leaves behind OVs. This migration is anisotropic. The OV injection only takes place along the $a_{M}\left(c_{R}\right)$ axis ${ }^{63,104}$. With enough $\mathrm{OVs}$, the $\mathrm{VO}_{2}$ transitions from insulating to metallic. However, the lattice remains monoclinic but with a $3 \%$ lattice expansion along the $a_{M}\left(c_{R}\right)$ axis ${ }^{62,63,82,99}$. The $\mathrm{VO}_{2}$ is now in a monoclinic metallic (MM) phase that is stable over long periods of time. The lattice expansion decreases the $\mathrm{V}-\mathrm{V}$ orbital overlap, decreasing the $\mathrm{d} / /$ band splitting. Also the loss of Oxygen leads to charge redistribution and an increase in carrier density; $O_{o}^{x} \leftrightarrow$ $V_{\ddot{O}}+2 e^{\prime}+\frac{1}{2} O_{2} \uparrow^{82,99,100}$. The effect of the OVs on the monoclinic phase can be seen in figure 1.5b. UV light has also been used to demonstrate doping of $\mathrm{VO}_{2}$ with OVs to generate metallicity ${ }^{100} . \mathrm{VO}_{2} \mathrm{OVs}$ are estimated to have an activation energy of 3-3.5 eV ${ }^{105,106}$ thus high energy UV light is needed for OV generation. Light below $3 \mathrm{eV}$ does not show the same effects ${ }^{100}$. 


\section{Chapter 2}

\section{Scanning Probe Imaging Modes \& Designing a Home-built SNOM}

Chapter 2 is dedicated to the home-built SNOM that is responsible for the vast majority of the experimental work done on this project. The primary experimental techniques performed with this instrument along with unique design features are highlighted. A SNOM is the combination of an Atomic Force Microscope (AFM) and table top optics. The SNOM cannot exist without both portions. AFMs are mature, scanning probe based instruments with a wide range of different imaging modes for characterizing a variety of material properties such as topography, electric domains, magnetic domains, and surface roughness. This chapter will start by reviewing fundamental working principles of AFMs. Next more sophisticated AFM functionalities, scanning probe lithography and Kelvin Probe Force Microscopy (KPFM), are introduced because these AFM modes are utilized for $\mathrm{VO}_{2}$ phase manipulations and detections. Then optics will be incorporated with the AFM to upgrade the instrument to a SNOM that captures optical information. Near-field optical microscopy is discussed along with the homebuilt instrument features. The necessity of the optics requires design modification compared to most commercial AFMs. Design specifics are discussed in section 2.4. Lastly, all the mentioned imaging modes are demonstrated simultaneously. In situ measurements of SNOM and KPFM are accomplished for the first time. This new imaging mode demonstrates the capability to correlate optical and electrical properties with nanoscale spatial resolution.

\subsection{Atomic Force Microscopy}

An AFM is a versatile tool for sample surface classification with sub-angstrom out-of-plane resolution by giving quantitative analysis of local forces. The main working principle of an AFM is to measure the force between a sharp probing tip and sample surface while raster scanning one of them relative to the other depending on instrument design ${ }^{107}$. This sequence systematically provides a 3D image of the force applied spatially throughout the sample surface. These force measurements have the versatility of working on conducting or insulating materials, which is unique compared to other scanning probe instruments such as a scanning tunneling microscope

108 . The sharp probing tips used are known as cantilevers, and they come in a variety of shapes and 
sizes. For the purpose of this dissertation, cantilevers will be restricted to a basic cantilever design where there is a large chip with a rectangular beam out of one end, and at the end of the beam is a sharp cone shaped tip with apex radius of approximately $10 \mathrm{~nm}$ depending on the make and model. The size of the tip apex radius defines the in-plane spatial resolution, and the nanoscale resolution is one of the advantages of AFMs. A diagram of a typical cantilever is shown in figure 2.1.

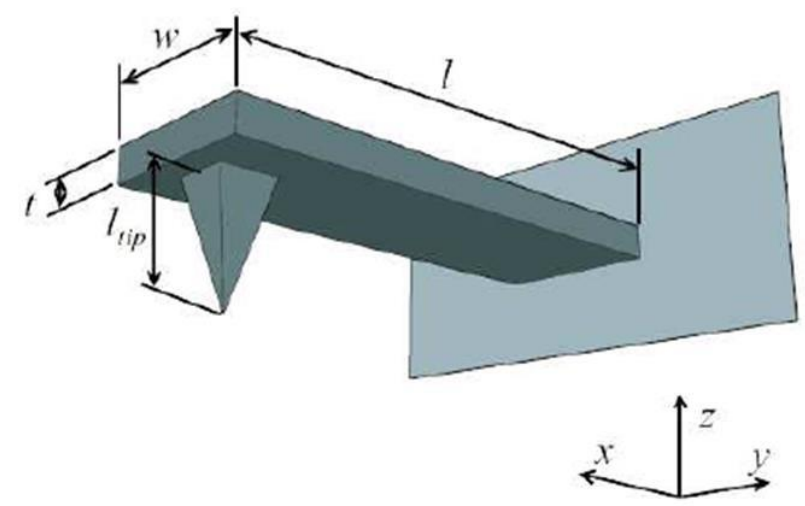

Figure 2.1 Illustration of AFM cantilever. Design of a typical AFM cantilever. ${ }^{108}$

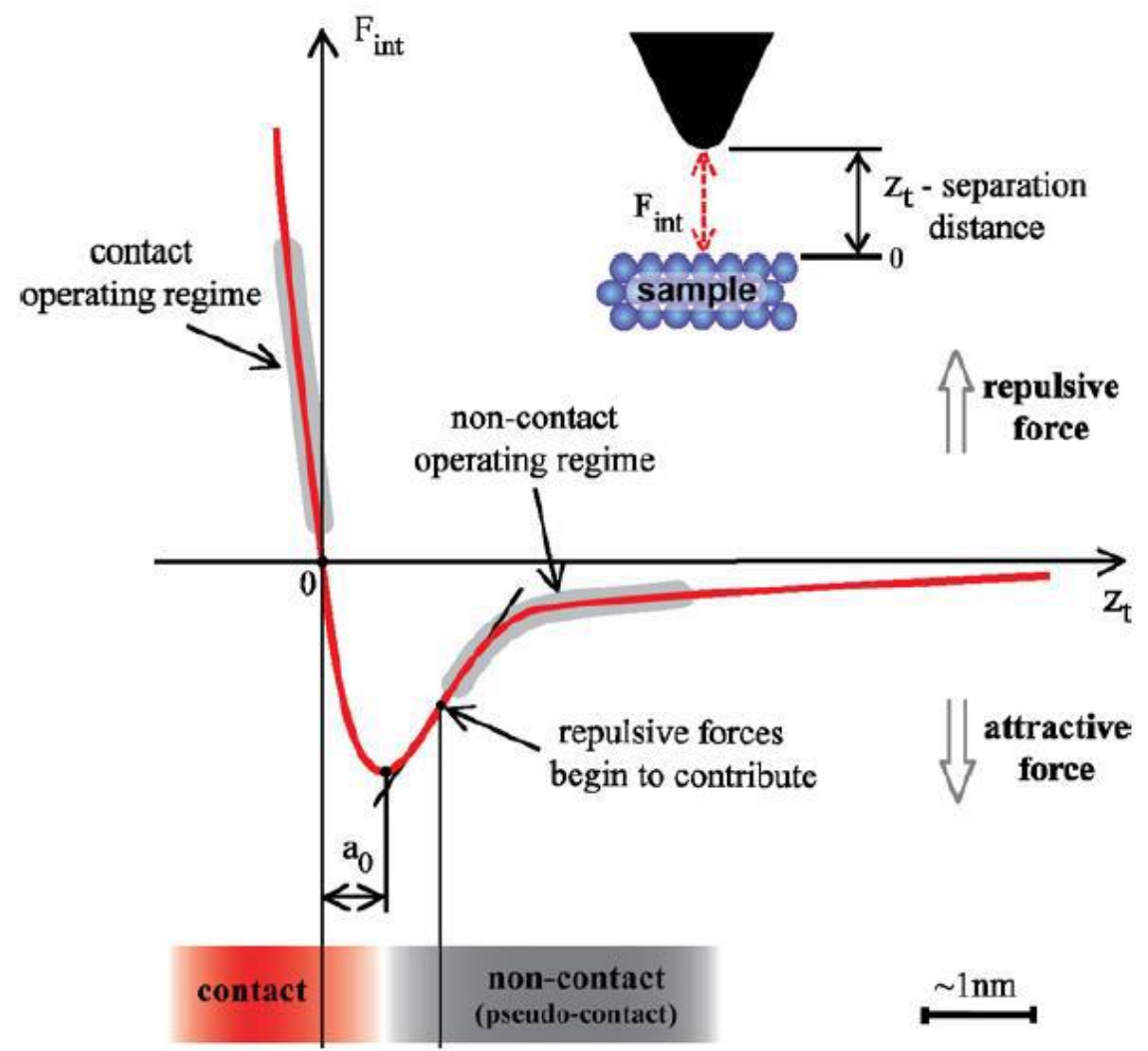

Figure 2.2 AFM force vs distance approach curve 108 
The beam of the cantilever has an associated spring constant, $\mathrm{k}$, and resonant frequency. This spring constant is the key in quantifying the forces between the tip and sample by using Hooke's Law, $\mathrm{F}=-\mathrm{k}^{*} \mathrm{z}$. By knowing the distance that the cantilever is displaced and the spring constant, the force between the cantilever and sample surface can be calculated. As the cantilever is moved closer and closer to the sample surface it primarily is responding to van der Waals forces. Figure 2.2 shows the force experienced by a cantilever as it approaches the sample surface. At distances far from the sample surface, the cantilever experiences no interaction with the sample. As it moves closer, the cantilever experiences an increasingly attractive van der Waals force until the tip and sample are brought into physical contact.
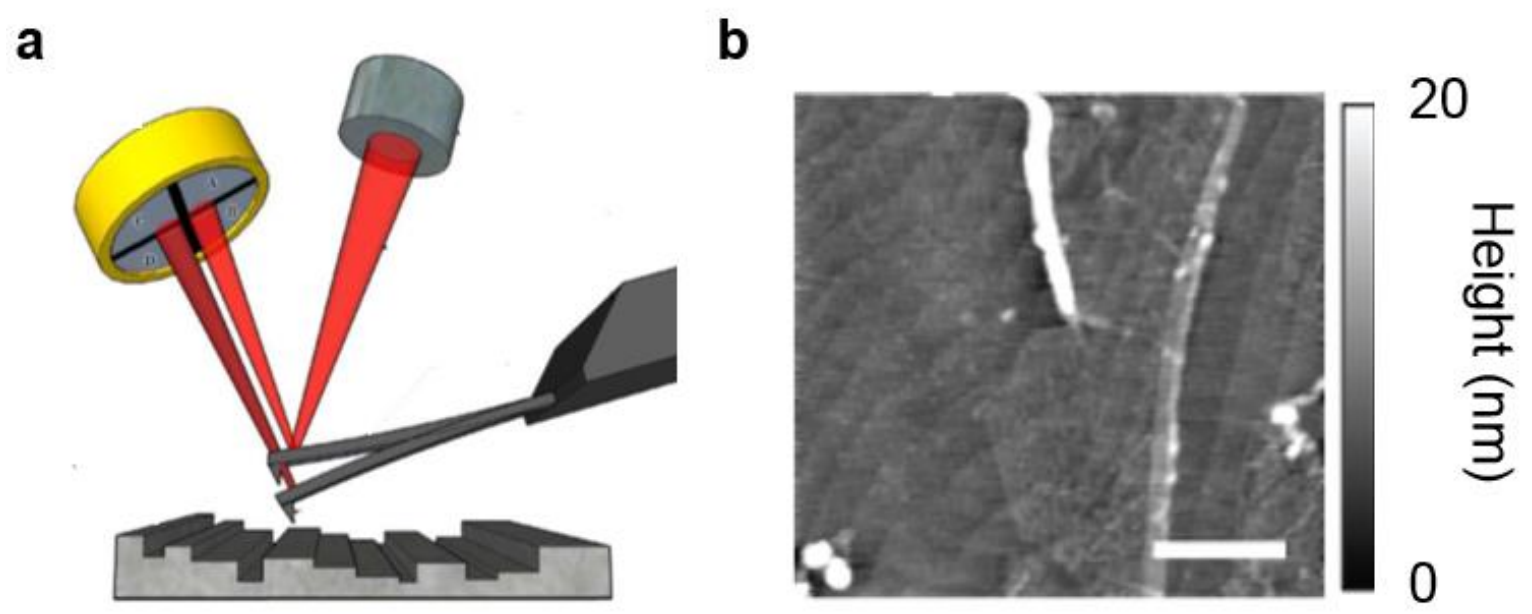

Figure 2.3 Illustration \& example of AFM measurement (a) Typical AFM measurement setup. 1. Cantilever interacts with sample surface. 2. Laser deflected from the back of the cantilever and collected in a segmented detector to equate displacement. 3. Feedback sent to either sample or tip to raise/lower to maintain cantilever set point ${ }^{108}$. (b) Example AFM topography image taken in noncontact mode with home-built AFM. Sample is graphene on SiC. SiC is comprised of terraces as can be seen in the image. Scale bar represents $1 \mu \mathrm{m}$.

When changes to the force between the sample surface and tip takes place, the cantilever is displaced up or down. AFMs measure the force by measuring this displacement. This is done by focusing a laser on the back of the cantilever beam and placing a four quadrant photodetector in the path of the reflected light. As the cantilever is displaced by topography changes for example, the laser spot is moved within the segmented detector. The relative intensity reading in each 
quadrant of the detector is the AFM measurement and feedback mechanism while raster scanning (figure 2.3a). The feedback tells the sample or tip to either raise or lower to maintain a preset, constant force on the cantilever. The home-built AFM in this work runs off a different measurement technique which will be discussed in section 2.4.

An AFM has two primary modes of operation, contact and noncontact. In contact mode, the cantilever tip is brought into contact with the sample surface in the repulsive force regime, and a raster scan is begun. As the cantilever tip is displaced up or down by topography as shown on the photodetector in figure 2.3a, a feedback loop raises and lowers the sample to maintain a constant force as the raster scan is performed ${ }^{109}$. This mode of operation creates high resolution topography images of the sample surface by recording how far the sample had to raise and lower at each point of the pixelated raster scan. The second primary mode of operation, noncontact mode, involves oscillating the cantilever tip at the designed resonant frequency using a piezoelectric actuator. The oscillations can be produced by other stimuli, but piezoelectric actuators is the most common method. The oscillations have an amplitude, phase, and frequency describing the motion of the cantilever ${ }^{110}$. Interaction with the sample will effect these properties providing information on the interaction. The oscillation initially has free air amplitude as seen by the photodetector and measured by a lock-in amplifier. As interaction occurs, the oscillation amplitude decreases. A set point is given for the desired amplitude of oscillation while scanning. Then similar to contact mode, a raster scan proceeds, and the sample is moved up and down by a feedback loop that maintains constant amplitude of the cantilever oscillation. Tapping mode offers some advantages to contact mode. One is that there are three potential quantities for operation of the feedback loop; amplitude, phase, and frequency ${ }^{110}$. All three are affected by the interaction of the tip and sample surface and can be measured simultaneously. A second advantage is that less degradation occurs to the tip and sample surface. The tip is not in constant contact with the sample surface in this operating mode ${ }^{110}$. An example topography image taken in noncontact mode with the home-built system is shown in figure $2.3 \mathrm{~b}$. The image is taken of a single layer of graphene on $\mathrm{SiC}$. The $\mathrm{SiC}$ terraces are clearly visible in the image and demonstrate the picoscale resolution. 


\subsection{Scanning Probe Lithography}

An AFM is typically known as an instrument that is used for measuring a variety of forces or surface features. However, it can also be used as a lithography style of tool. The AFM tip can be used for material manipulations. The application and future of nanotechnology requires the capability to fabricate the desired nanostructures. Nanoscale spatial resolution and positioning with versatile material compatibility has proven difficult ${ }^{111,112}$. Many lithography techniques have been developed or modified thus far for nanotechnology such as electron-beam lithography, photolithography, nanosphere lithography, and ion beam lithography ${ }^{113-117}$. Electron-beam is the most often used technique currently. It is capable of the required resolution but is very time consuming which limits its commercial application. Also it requires a resist to be placed on the sample surface which can leave residue behind and harm sample quality or device performance. This has proven detrimental to research with 2D materials such as graphene ${ }^{116,118}$.

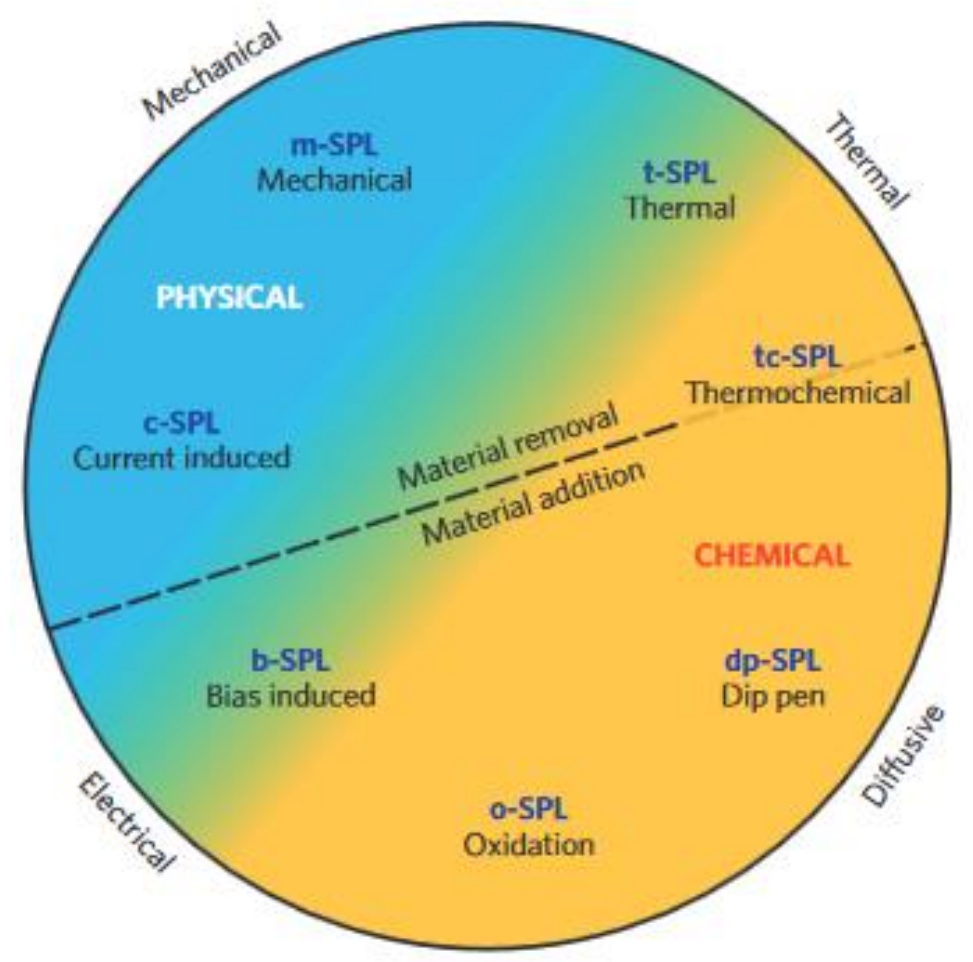

Figure 2.4 Diagram of various scanning probe lithography methods. Classification of SPL methods according to the dominant tip-surface interaction used for patterning. Broken down into 4 categories; electrical, thermal, mechanical, and diffusive processes. ${ }^{111}$ 
Recently AFMs are gaining more attention as a lithography tool. Scanning probe lithography (SPL) has emerged as a resist-free, single-step lithographic technique with nanoscale spatial resolution. It has low technological requirements being performed in ambient conditions and even has the ability to handle soft matter and 2D materials ${ }^{111,118,119}$. The basic principle of SPL is to use the scanning probe to locally perform a material modification. The probe can maneuver in designed patterns and has spatial resolution set by the radius of the probe apex, $\approx 10 \mathrm{~nm}$. SPL techniques are primarily divided into two main categories; material removal and material addition. Figure 2.4 subdivides these two divisions into many of the different SPL techniques that have been demonstrated thus far such as oxidative, dip pen, thermal, and mechanical ${ }^{111}$. These different techniques have successfully demonstrated various localized material modifications on silicon compounds $^{120,121}$, polymers ${ }^{122} 123{ }^{124}$, proteins ${ }^{125}$, ferroelectrics ${ }^{126}{ }^{127}$, and 2D materials such as graphene 128129130131132 , phosphorene ${ }^{133}, \mathrm{MoS}_{2}{ }^{134135}$, and $\mathrm{WSe}_{2}{ }^{136}$.

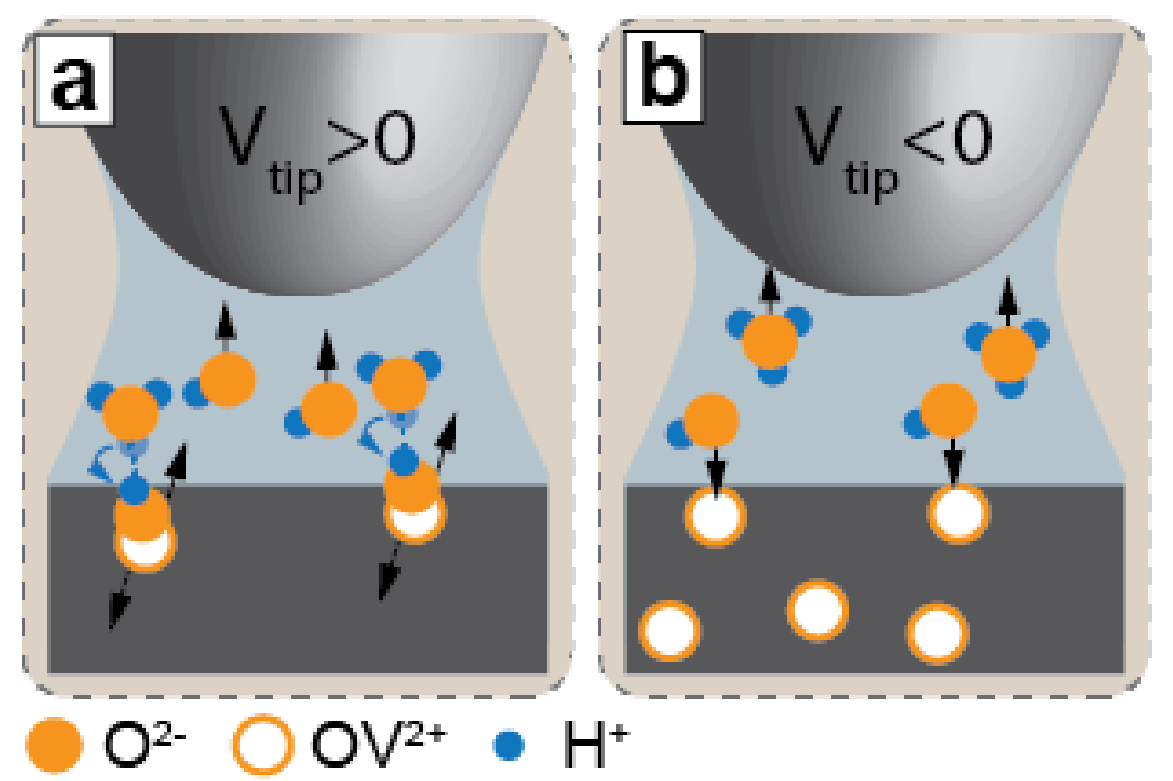

Figure 2.5 Illustration of reduction and oxidation SPL (a) Reduction SPL pulls oxygen ions out of the sample using an applied tip bias. (b) Oxidation SPL forces oxygen from the atmosphere into the sample using an applied tip bias.

In the following chapters, I will be working with both material removal and addition on strongly correlated $\mathrm{VO}_{2}$ thin films. The scanning probe is used to apply different forms of stimuli with nanoscale resolution. In one experiment strain is applied by the scanning probe by pressing 
down on the film with increased force. As explained in chapter 1, compressive strain lowers the $\mathrm{T}_{\mathrm{c}}$ of the $\mathrm{VO}_{2}$ IMT. The primary phase transition of this project is induced by using the scanning probe to supply an electrical bias between the tip and sample. In samples such as $\mathrm{SiO}_{2}{ }^{120,121}$, $\mathrm{MoS}_{2}{ }^{134135}$, and graphene ${ }^{128} 129{ }^{130}$, it has been found that oxygen stoichiometry can be altered. A water layer on the film surface can be ionized by the local electric field under the cantilever tip. These Oxygen ions then migrate towards the film surface to oxidize the sample. This is known as oxidation SPL by the lithography community. If the bias is reversed, Oxygen can then be pulled out of the film leaving behind Oxygen Vacancies; $O_{o}^{x}=V \ddot{o}+2 e+\frac{1}{2} O_{2} \uparrow 100$. This is known as reduction SPL. These lithography styles are similar to the electrolyte gating discussed in chapter 1, except now the electrolyte gel is replaced by the water layer naturally on the film surface. A schematic of reduction and oxidation SPL are shown in figure 2.5.

\subsection{Kelvin Probe Force Microscopy}

As discussed in section 2.1, AFMs measure the force between the tip and sample. In the most fundamental measurement modes, these are van der Waals forces. However, other forces can also be measured such as Coulomb interactions. AFM's are increasingly used to diagnose electric potentials of material surfaces while simultaneously measuring the topography.

Rather than mechanically oscillating the cantilever at its resonant frequency using piezoelectric actuators, oscillations can also be accomplished by driving with an AC bias. In this scenario, the cantilever oscillation amplitude is affected by the electric field between the tip and sample which depends on the local electrostatic properties of the sample. Raster scanning and mapping out the change in cantilever oscillation allows an image to be created that is based on the local surface potential and work function of the sample. This imaging mode is known as electrostatic force microscopy and is the simplest of the electrostatic force probing modes ${ }^{137}$. However, it only obtains qualitative changes to the sample work function. KPFM is an electrostatic force microscopy mode that improves upon the qualitative results and makes them quantitative $^{138,139}$. A feedback loop nullifies the electric field between the tip and sample by supplying a DC bias between them. The base idea was first conceived by Lord Kelvin in 1898 using two metal discs ${ }^{140}$. 
There are two primary styles of KPFM that will be discussed; amplitude modulated (AM) and frequency modulated (FM). AM-KPFM was implemented in $1991^{139}$ and is the more common of the two modes for measuring the electrostatic force.

$F_{e l}=\frac{1}{2} \frac{\partial C}{\partial z} U^{2}$

$U=U_{D C}-\frac{\Delta \varphi}{e}+U_{\text {mod }} \cos \left(\omega_{\text {mod }} t\right)$

The force between the tip and sample is comprised of three terms (2.3.2). UDC is the DC bias supplied between the tip and sample. $\Delta \varphi$ is the local contact potential difference between the tip and sample. This is the difference of the work functions, and the naturally existing force. Then $\mathrm{U}_{\text {mod }}$ is an AC bias supplied for modulating the cantilever. Equation 2.3.1 and 2.3.2 together can be separated into three terms based on their modulation frequency.

$F_{e l}=\frac{1}{2}\left[\left(U_{D C}-\frac{\Delta \varphi}{e}\right)^{2}+\frac{1}{2} U_{m o d}^{2}\right] \frac{\partial C}{\partial z}+\frac{\partial C}{\partial z}\left(U_{D C}-\frac{\Delta \varphi}{e}\right) U_{m o d} \cos \left(\omega_{m o d} t\right)+$

$\frac{1}{4} \frac{\partial C}{\partial z} U_{\text {mod }}^{2} \cos \left(2 \omega_{\text {mod }} t\right)$

In AM-KPFM, experimentally one can modulate the cantilever $\left[\mathrm{U}_{\bmod } \cos \left(\omega_{\bmod } \mathrm{t}\right)\right]$. The cantilever oscillation amplitude is demodulated by a lock in amplifier at $\omega_{\text {mod. }}$ Next a DC bias is used to nullify the amplitude of the oscillation; $\left(\mathrm{U}_{\mathrm{DC}}=\Delta \varphi / \mathrm{e}\right)^{141142143}$. As the raster scan takes place, the feedback loop continuously adjusts $\mathrm{U}_{\mathrm{DC}}$. This maps the work function of the sample surface relative to the tip work function. It is a quantitative map of the work function. Typically the electric modulation operates on the $2^{\text {nd }}$ harmonic of the cantilever while the $1^{\text {st }}$ harmonic is reserved for noncontact topography measurement. Both are run in situ.

Some experimental setups choose to use a dual pass, lift off method. First the topography is measured on the first harmonic. Then the cantilever is lifted by 10-50 nm and scanned again. This time only electric modulation takes place but it can also be at the $1^{\text {st }}$ harmonic. This allows for a stronger modulation due to the AC bias. In this method, topography and electrostatic forces are more decoupled since they are run on separate passes, and the liftoff removes topography influence. The drawback of this dual pass method is that it requires longer scan times and a loss of spatial resolution due to the diverging electric field with liftoff distance. Overall AM-KPFM is reliable and simple to implement. However, the force between the tip and sample is not completely 
localized to the tip apex and sample surface as commonly depicted. The large metallic body of the cantilever itself influences the measurement with a background contribution ${ }^{142,143}$. The force from all the metal components each have a $1 / \mathrm{z}$ distance dependence.

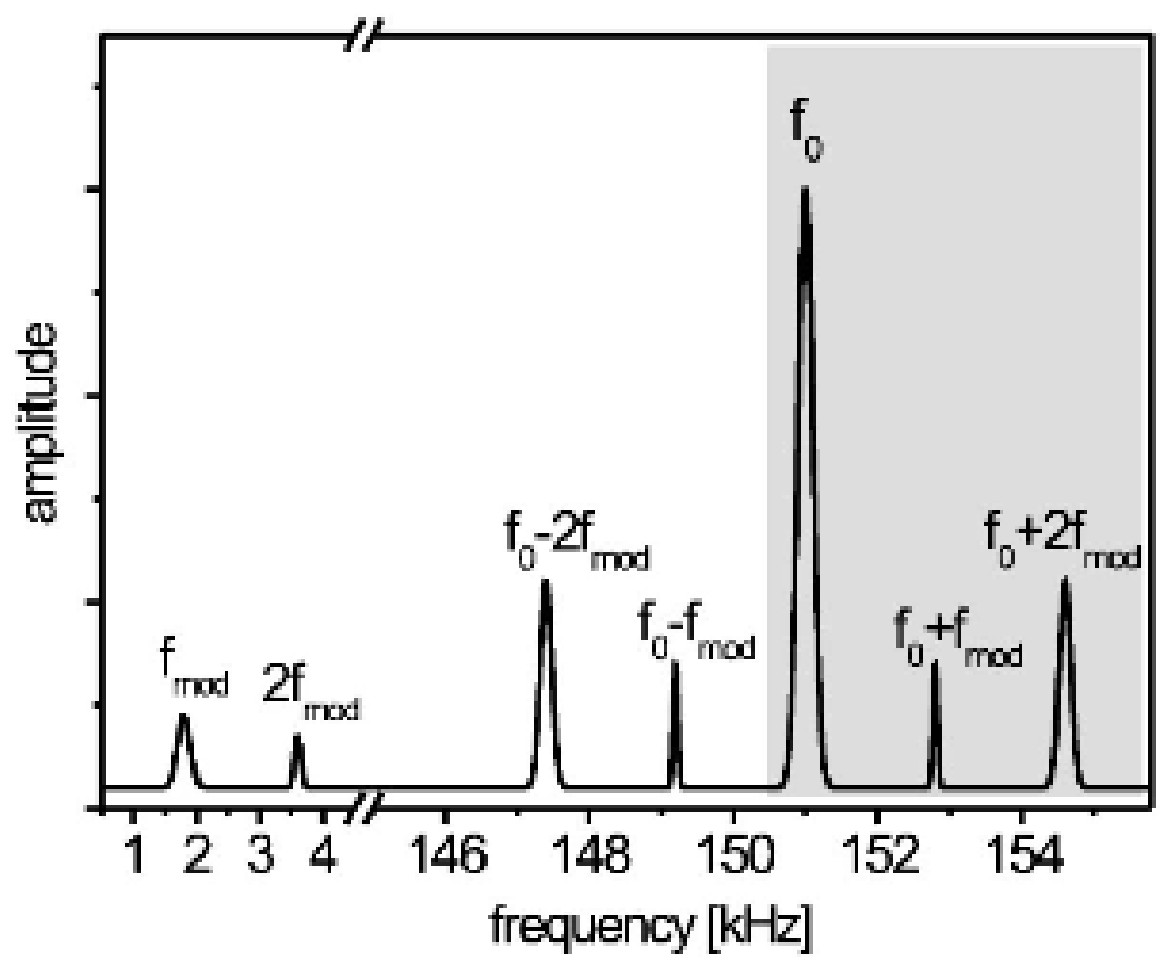

Figure 2.6 FM-KPFM frequency bands. Frequency spectrum of the cantilever oscillation. An applied AC bias is responsible for the modulation frequencies. The gray region represents the experimentally used spectrum for FM-KPFM. ${ }^{141}$

This non-localization of the electric field leads to the major advantage of FM-KPFM, reducing the influence of the cantilever body on the overall measurement. The force gradient is measured instead of the force ${ }^{142,143}$ which provides a much greater distance dependence. This makes the tip apex influence dominant over the cantilever body. In AM-KPFM, the modulation voltage was applied at a cantilever resonance. FM-KPFM supplies a lower frequency modulation voltage, typically $\omega_{\text {mod }} \approx 5 \mathrm{kHz}$. The force gradient ( $\frac{\partial F}{\partial z}$ ), will create side bands off of the resonant frequency based on this modulation frequency as shown in figure 2.6.

$f_{0}^{\prime}=\frac{1}{2 \pi} \sqrt{\frac{k-\partial F / \partial z}{m^{*}}} \approx f_{0}\left(1-\frac{1}{2 k} \frac{\partial F}{\partial z}\right)$ 


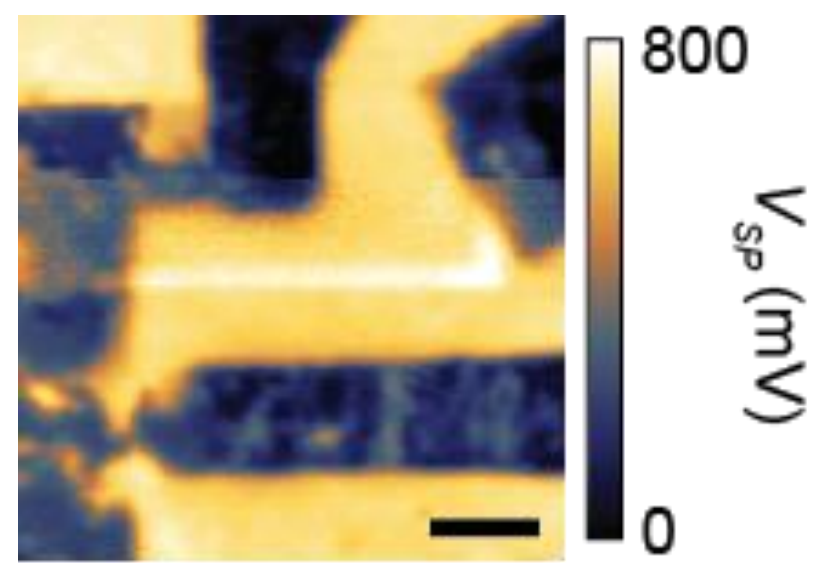

Figure 2.7 Example AM-KPFM image. AM-KPFM image obtained of Au electrodes and graphene on top of LAO/STO. The dark regions represent both conductors, Au and graphene. The yellow/orange region is the insulating LAO/STO. The bright white channel is a written 2DEG channel in the LAO/STO interface. Scale bar represents $10 \mu \mathrm{m}$.

Demodulation then takes place at the side band $\mathrm{f}_{0}+\mathrm{f}_{\text {mod, }}$, where the oscillation amplitude is nullified in the same manner as AM-KPFM (2.3.3). The topography can still be mechanically measured at $f_{0}$ provided $f_{\text {mod }}$ is larger than the bandwidth of $f_{0}$. The force gradient is not sensitive to the body of the cantilever as the gradient creates a $1 / \mathrm{z}^{2}$ dependence. The cantilever body is much further away than the tip making the FM method a truer measurement of surface potential and with greater spatial resolution ${ }^{141} 142143$. An example KPFM surface potential image of Au electrodes and graphene on LAO/STO is shown in figure 2.7. The dark regions represent both conducting graphene and Au electrodes. The yellow/orange areas are the insulating LAO/STO background. Also the bright white channel is a written 2DEG channel in the LAO/STO interface. This image was taken in AM-KPFM mode. Example FM based images will be shown in section 2.5.

\subsection{Scanning Near-field Optical Microscopy \& Instrument Design}

Chapter 2 thus far has entirely focused on AFMs and their strengths such as nanometer spatial resolution. However, the biggest drawback of AFMs has not been discussed. They do not obtain any optical information about a sample. "Light is our primary source of information about the environment which surrounds us ${ }^{144}$." It provides the fingerprints of the materials it interacts with. Conversely many optical experiments are limited by spatial resolution. The diffraction limit of light limits the spatial resolution to approximately $\lambda / 2$, while atomic distances are typically on the 
order of a few angstroms ${ }^{145}$. Thus the area under investigation in most optical experiments are much greater in size than the nanoscale domains of many materials.

SNOM has emerged in the new millennium as an optical technique that breaks the diffraction limit of light ${ }^{146}$. This technique combines two well-known instruments, a scanning optical microscope and an AFM. A brief overview is that light is focused from the optical microscope onto the metallic tip of the AFM which confines the light proportional to the size of the tip apex radius of curvature and within the near-field approximation of light-matter interaction. The light can then interact with the sample surface with spatial resolution that is wavelength independent and determined by the AFM tip ${ }^{144}$. Then the scattered light from the near-field interaction is collected and measured during a raster scan. SNOM is the optical property imaging mode of an AFM. It was first experimentally realized in the 1980's when 2 different groups developed 2 aperture based SNOM systems. Then in the 1990's s-SNOM (scattering type SNOM) was successfully demonstrated which is the vast majority of today's SNOM setups ${ }^{147}$.

The primary purpose of engineering the home-built AFM in this work was to gain the SNOM imaging mode. Throughout this section the operational setup, SNOM imaging mode, and design customization will all be discussed. First the table top optics within the scanning optical microscope will be discussed. This is the primary operational setup difference between a commercial AFM and SNOM setup, the addition of table top optics. A diagram of my optical table is shown in figure 2.8. The optical table begins with two light sources. The primary one used for SNOM is a Daylight Solutions quantum cascade laser operating in the MIR regime. This laser offers up to $80 \mathrm{~mW}$ of power and is tunable between 10.4-11.2 $\mu \mathrm{m}$ where many material resonances exist such as surface plasmon polaritons ${ }^{148-150}$ and surface phonon polaritions ${ }^{151-153}$. Initially the light passes through a beam expander to make the beam diameter approximately 8 $\mathrm{mm}$. This allows for a higher numerical aperture focusing objective later in the optical setup. Also the optical path length is long, and beam divergence is inversely proportional to diameter ${ }^{154}$. The light is then used in a common Michelson interferometer setup. It passes through a beam splitter creating two arms; the main arm and reference arm. The main arm passing straight through the beam splitter is directly sent into a high vacuum chamber where the sample is housed. Inside the high vacuum chamber, the light is focused by an off axis parabolic mirror $(\mathrm{NA}=0.5)$ to the AFM tip. The only optical element inside the vacuum chamber is the parabolic mirror, and it is oriented 
with its optical axis $65^{\circ}$ from the sample surface normal allowing the maximum collection angle above the sample surface. The scattered light from the tip is collected by the same parabolic mirror and sent back through the interferometer. At the beam splitter, the collected light is now reflected to a $\mathrm{HgCdTe}(\mathrm{MCT})$ detector. The initial reflecting arm at the beam splitter is used as a reference arm for amplification and homodyne detection ${ }^{155} 156$. The reference arm is retro reflected by a mirror on a nano-positioning stage back to the beam splitter and to the MCT detector. The two arms are focused together to interfere at the MCT detector. The constant reference arm is used as a stable phase to measure phase changes in the SNOM optical signal. The interfering reference arm is also used to amplify the SNOM optical signal.

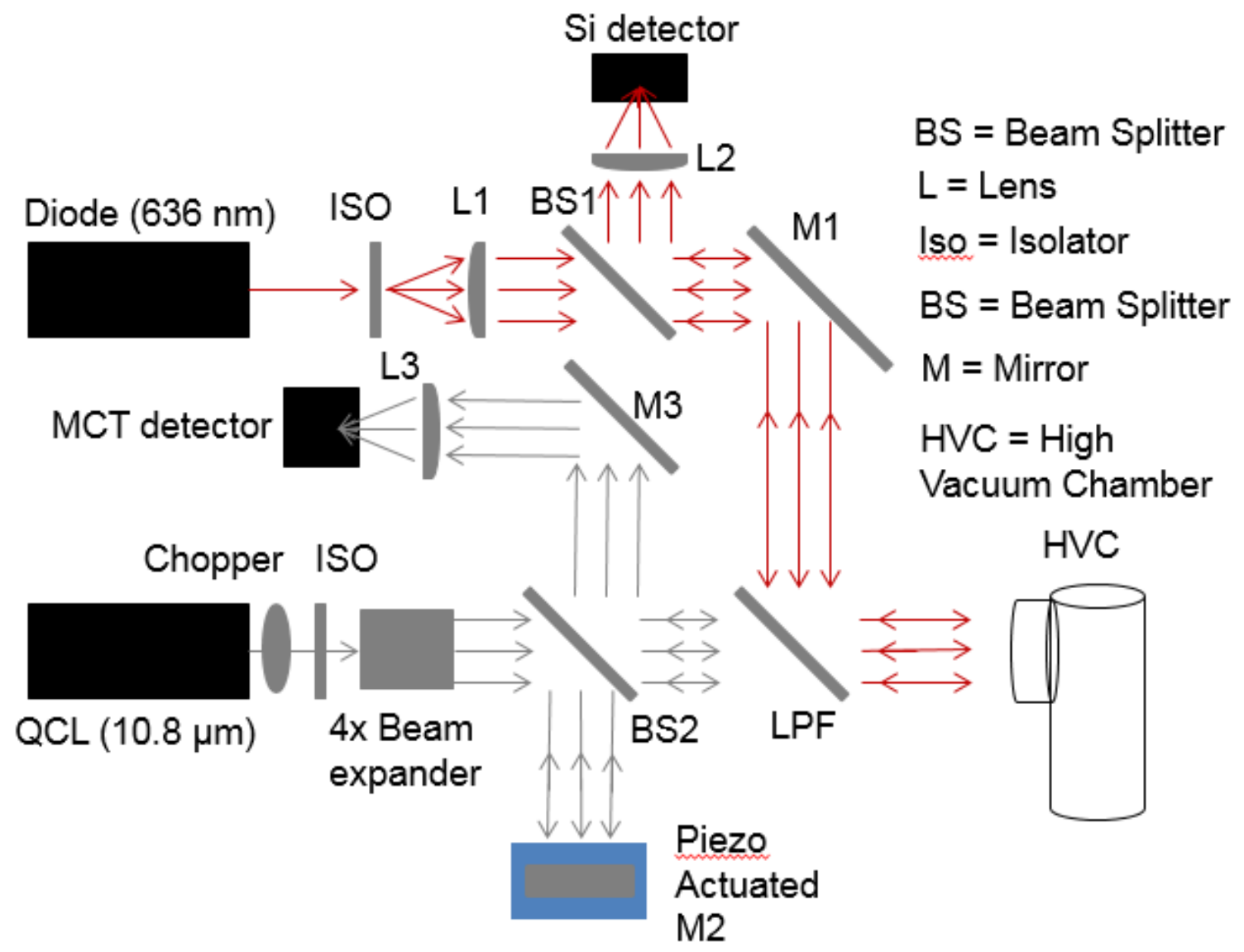

Figure 2.8 Diagram of SNOM optical table. Optical table diagram of SNOM setup. The table is equipped with two light sources; red diode laser and MIR QCL. Both are used in common Michelson interferometer setups where the light reflects from the sample surface, travels back to a beam splitter, and is directed to the proper photodetector. 
The second light source is a red diode laser with the same Michelson interferometry setup. Before light enters the vacuum chamber, a long pass filter is used to combine the two light sources. They are aligned to be parallel and coincident. Inside the vacuum chamber, the sample, AFM tip, and light focal point all need to reach the same interaction point. This has to be done by a high magnification camera and alignment of each element individually. The visible light is used for the alignment of the invisible MIR light. The entire goal is to focus the MIR light into this interaction point. It should be noted that SNOM can also be performed using the visible light as the operation is wavelength independent. Care needs to be taken as optical windows for the high vacuum chamber are sensitive to wavelength. A ZnSe window needs to be used to allow transmission of the MIR light, and the visible light can still be detected by the naked eye with only $\mu \mathrm{W}$ power.

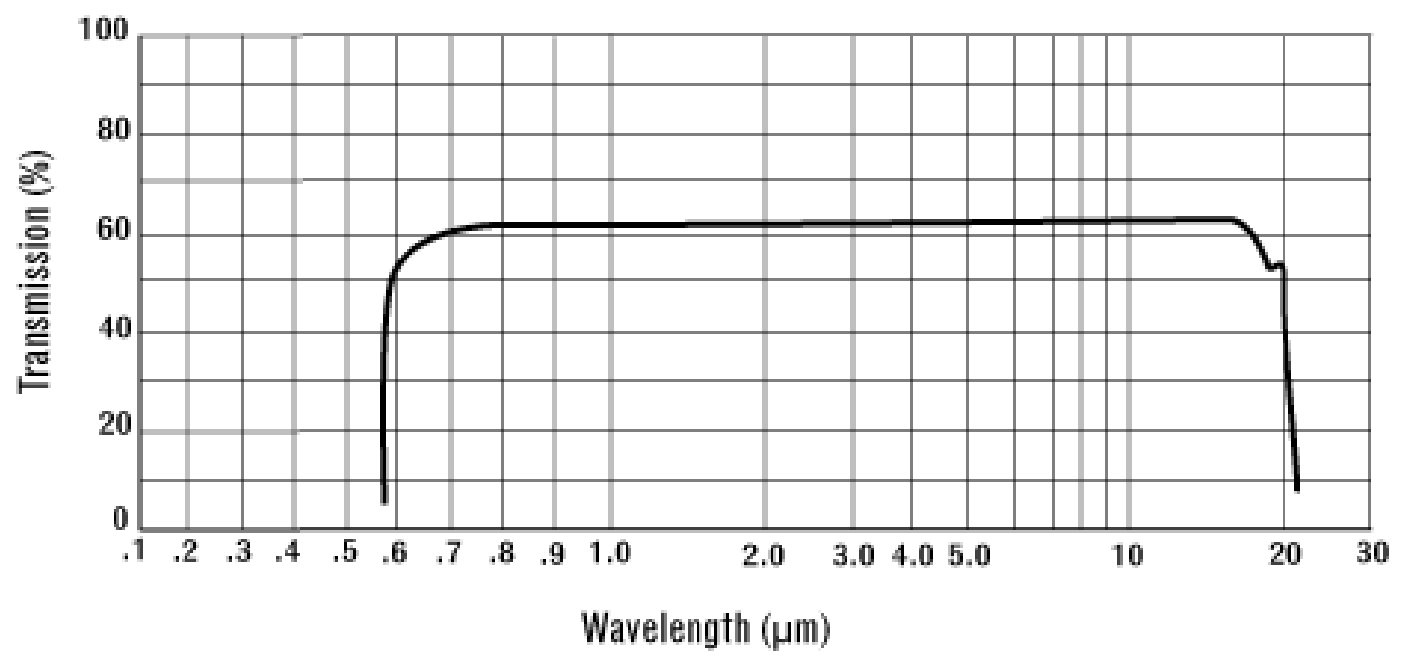

Figure 2.9 Transmission spectrum of ZnSe. Wavelength vs transmission for a ZnSe window. ${ }^{157}$

It is important to discuss further the homodyne background suppression scheme. Inside the vacuum chamber, light is being focused to the AFM tip. The focal point size of the light is fundamentally limited by the diffraction limit of light. It is on a micro scale while the tip size is nanoscale. A large percentage of the light reflects off other entities other than the tip such as the cantiléver body and sample. This light can also be collected and is known as background light. The background light over powers the near-field probing signal scattered only by the tip, and background suppression has to be performed to isolate the useful near-field signal. SNOM is run with the AFM in non-contact mode so the tip is actually oscillating above the sample surface at frequency $\omega$. It has an oscillation amplitude of a few tens of nanometers. Within this distance 
variation, the near-field signal is constantly changing as the tip oscillates due to the evanescent nature of the near-field interaction. The near-field signal has a nonlinear distance dependence. The near-field signal can be demodulated at higher harmonics due to this nonlinear dependence on the distance from the sample surface ${ }^{155}$. However the background signal is not evanescent and is essentially a constant while the tip is oscillating. Therefore the background signal will have minimal contribution at higher harmonics. By demodulating at higher harmonics, measurements of the gradient with respect to the distance are being taken. The background signal is constant and doesn't have this gradient. Figure 2.10 shows SNOM images taken of an Au electrode on LAO/STO when being demodulated at different harmonics of the oscillation frequency. The images are taken with an oscillation frequency of $72 \mathrm{kHz}$ and $10.8 \mu \mathrm{m}$ light.

a
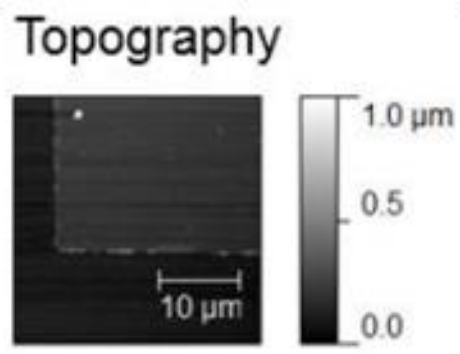

d
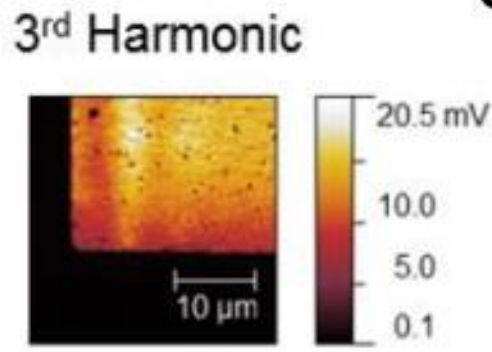

b $1^{\text {st }}$ Harmonic

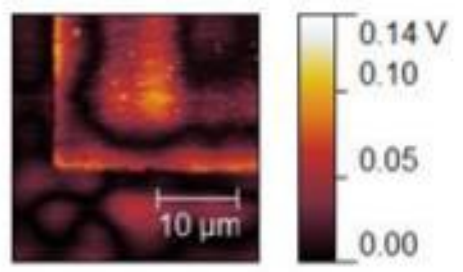

e $4^{\text {th }}$ Harmonic

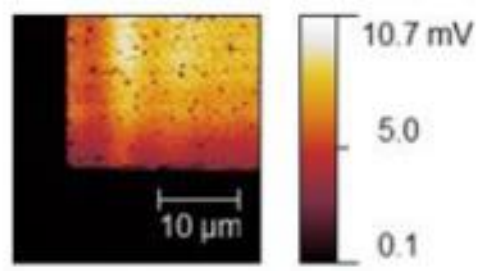

C $2^{\text {nd }}$ Harmonic

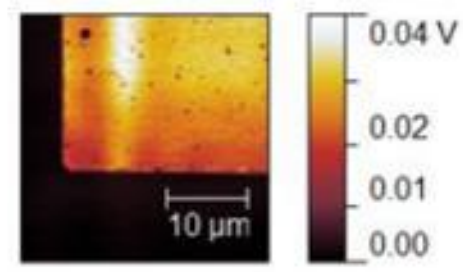

f $6^{\text {th }}$ Harmonic

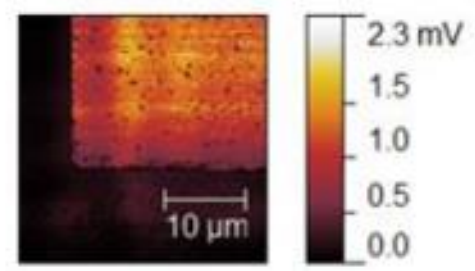

Figure 2.10 SNOM images of high harmonic background suppression. SNOM amplitude images of an Au electrode on LAO/STO taken with $10.8 \mu$ m light. (a) AFM topography (b,c,d,e,f) SNOM amplitude demodulated at $1^{\text {st }}, 2^{\text {nd }}, 3^{\text {rd }}, 4^{\text {th }}, 6^{\text {th }}$ harmonic.

The background effects can clearly be seen in the $1^{\text {st }}$ harmonic image where there is minimal background suppression. The image has optical artifacts compared to the higher harmonic images. At the $2^{\text {nd }}$ harmonic and higher the artifacts are removed, and the fringe patterns on the Au are surface plasmons reflecting off the Au edge. Further background suppression could be obtained 
by using a pseudoheterodyne detection scheme which involves a modulated reference arm for quantitative phase information. The setup I use throughout this project is a homodyne detection scheme which only consists of a stable, amplifying reference arm.

Thus far it has been discussed that in a SNOM setup light can break the diffraction limit due to the AFM tip acting as a nano-antenna, but not what quantities are actually being measured. Light scattered from the tip is carrying information about the sample surface. The metallic tip becomes polarized due to the incident light focused on it. The induced polarized charges in the tip are then what interact with the sample surface. It creates an electrostatic method of images style of problem as illustrated in figure $2.11^{158}$.

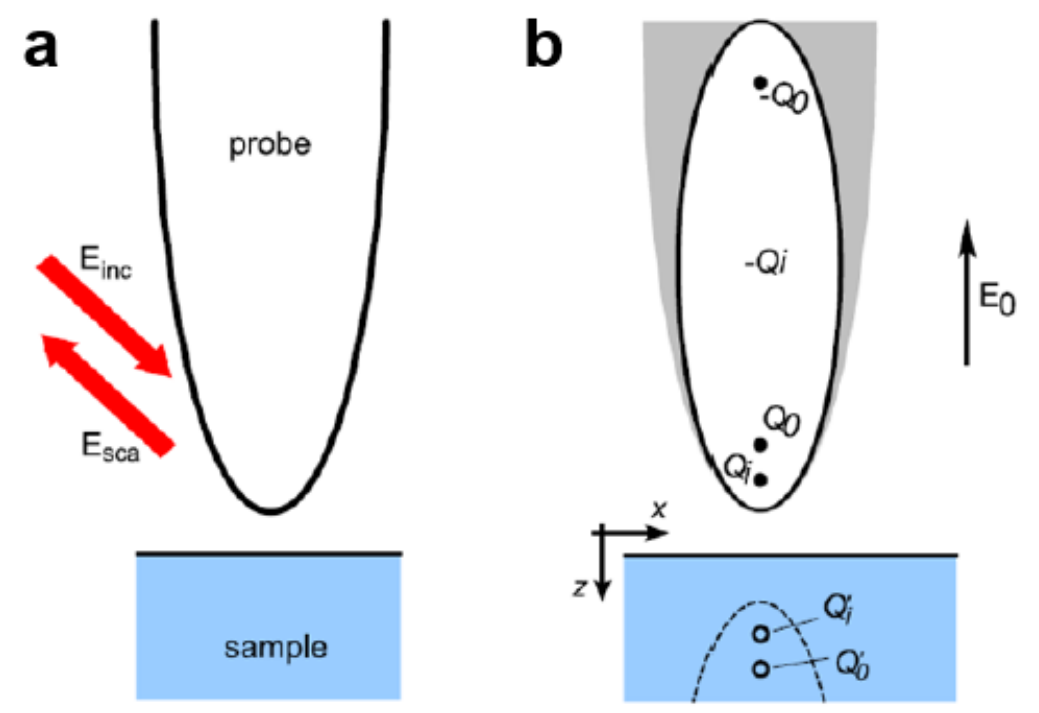

Figure 2.11 Finite dipole model diagram. (a) SNOM systems focus light to the AFM tip and then collect the scattered light. (b) Finite dipole model for the polarized spheroid tip interacting with the sample surface. ${ }^{158}$

This tip sample interaction is modeled as the finite dipole model where the tip is approximated as a dipole with only the close proximity charge interacting with the sample surface. The key points of this method of images problem need to be highlighted. The electric field induced charge in the tip $\mathrm{Q}_{0}$ interacts with the sample surface due to its close proximity. The interaction is based on the permittivity of the sample, and the image charge then acts back on the tip as shown in equation 2.4.1 and 2.4.2 $2^{158}$. 
$Q_{i}=\frac{-\beta f_{0}}{1+\beta f_{1}} Q_{0} \quad\left(f_{i}=\right.$ geometric factor $)$

$\beta=\frac{\varepsilon_{S}-1}{\varepsilon_{S}+1}$

Then this interaction needs to be seen in the scattered light for measurement. The tip is a radiating dipole in the incident light, thus scattering new light. The scattered light is based on the polarizability of the tip. If the sample was not present, the scattered light would be constant. However, the locally induced image charges effect the dipole moment of the tip and thus the scattered light in equations $2.4 \cdot 3-2.4 .6^{158}$. There is an effective polarizability. This is how the nearfield interaction between the tip and sample is probed using SNOM. The scattered light is a qualitative measurement of the dielectric function of the sample. An example SNOM image taken in the home-built setup is shown in figure 2.12. The sample is Au nano-cubes patterened on LAO/STO (left) and a thin Au electrode (right). The topography and SNOM images demonstrate the nanoscale spatial resolution. The SNOM image shows contrast between the Au and LAO/STO based on the dielectric function of the materials.

$$
\begin{array}{ll}
E_{s c a} \sim p=\alpha E_{0} & (\alpha=\text { polarizability }) \\
p=Q * d & (\mathrm{p}=\text { dipole moment }) \\
p_{\text {eff }}=p_{0}+p_{i} & \\
E_{\text {sca }} \propto \alpha_{\text {eff }} E_{\text {inc }} &
\end{array}
$$
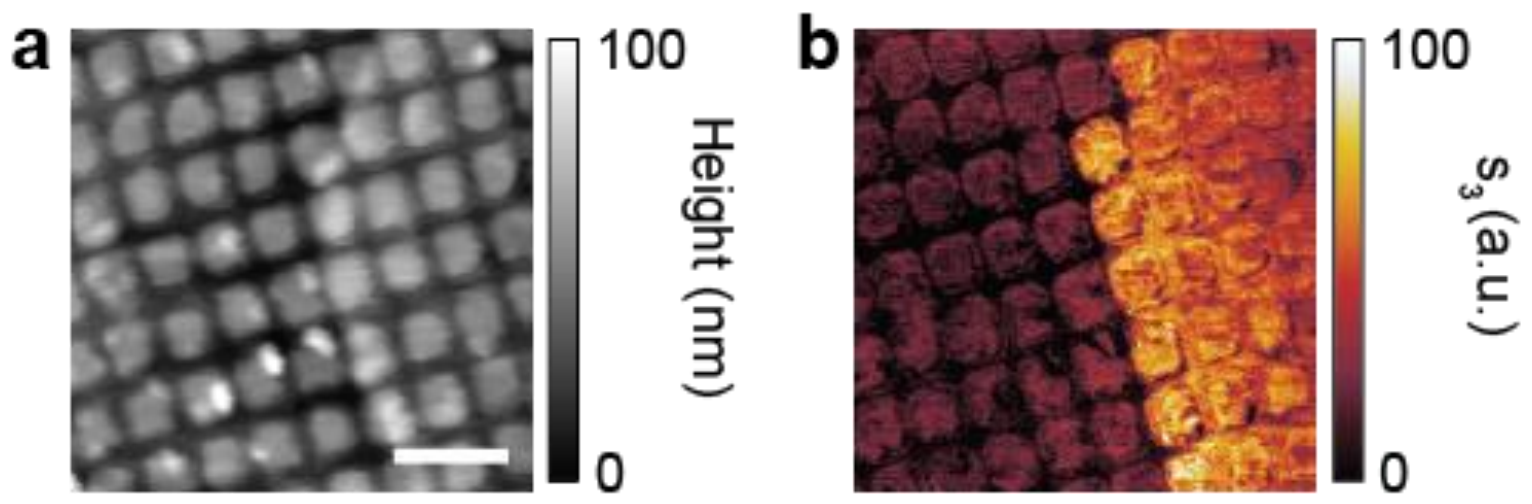

Figure 2.12 Example SNOM image. (a) AFM topography image of Au nano-cubes. (b) SNOM image of Au nano-cubes demodulated at the third harmonic. Left region are Au nano-cubes on LAO/STO. Right region is Au nano-cubes on Au. Scale bar represents $500 \mathrm{~nm}$. 
Transforming an AFM into a SNOM seems simple experimentally, buy a commercial AFM and focus light on the AFM tip. However, there are design requirements such as the AFM tip needs to have an open optical path, and a maximized numerical aperture and collection angle are necessary to improve the signal to noise ratio. This home-built SNOM was designed with numerous features to maximize the instrument capability. First the sample stage is equipped with both coarse and fine positioning systems. A piezo scanner controls fine motion during imaging. Also coarse positioning motors comprised of strike-slip piezo actuators control 3D positioning. The PCB board that holds the sample also has a 28 pin connection system for the capability of any variety of electrical biasing. Numerous styles of PCB boards have been built for different samples. A Solidworks representation of the sample stage and holder can be seen in figure 2.13a.

Next the AFM tip has to be added with similar 3D positioning control while maintaining an exposed cantilever. The AFM system is modeled in figure 2.13b. The overall spatial design is abnormal. A wheel that sits in a tower (not shown) holds a thin, stem-like structure. This design exposes the cantilever to external light for $180^{\circ}$. Thus it also increases the possible collection angle of scattered light. Also there are no spatial constraints for additional external optics. This body style minimizes the AFM spatial footprint. At the top of the stem, strike-slip piezo actuators supply the $3 \mathrm{D}$ tip positioning. The top of the wheel allows easy access for necessary electrical connections for the AFM.

The entire system, sample stage and AFM, is designed to be vacuum compatible. As mentioned previously, all parts are housed within a high vacuum chamber capable of experiments at $10^{-5}$ mbar. A resistive heater is attached beneath the sample to heat $\mathrm{VO}_{2}$ samples above the IMT transition temperature, and the system is designed with attachments for a cryostation. A cold finger may be attached beneath the sample in the hollow sample positioning stages to allow for future low temperature measurements. 

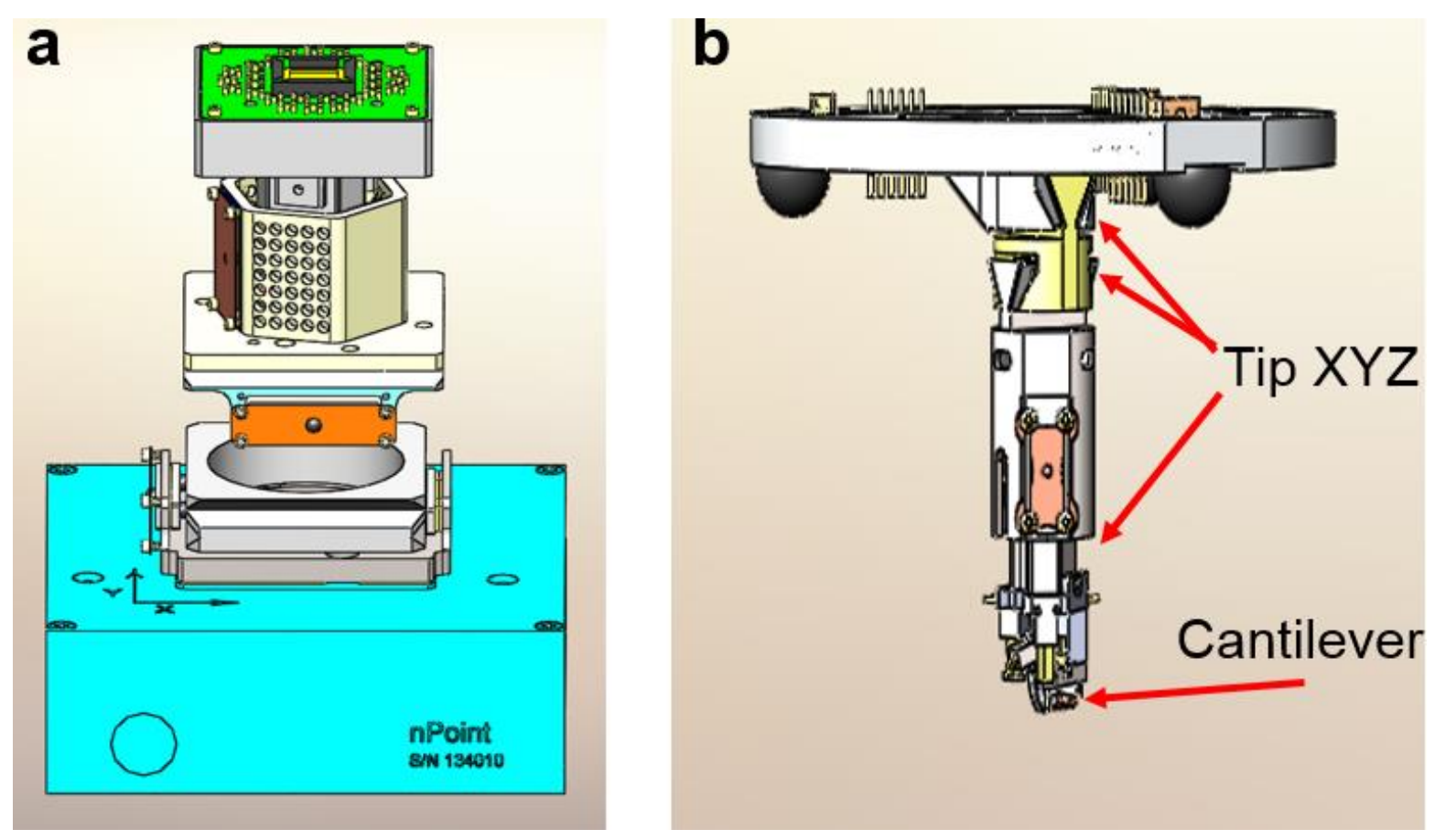

Figure 2.13 SNOM custom design schematic (a) Solidworks drawing of sample holder and positioning stage. (b) Solidworks drawing of AFM.

One of the common spatial constraints encountered when transforming an AFM into a SNOM is housing the feedback system. As discussed in section 2.1, a laser and detector are needed to measure the deflection of the cantilever. These elements require large spatial footprints. This home-built SNOM system utilizes a different style of feedback system based on interferometry. Figure 2.14 shows the Solidworks model for the flexure interferometer feedback system. Single mode, $1310 \mathrm{~nm}$ light is delivered to the back of the cantilever by an optical fiber. The optical fiber is angled normal to the cantilever so that the reflected light from the cantilever is collected by the same optical fiber. The optical fiber network includes a circulator so that the collected light is directed to a photodetector rather than back to the light source. The end of the optical fiber at the cantilever is flat cut with no antireflection coatings so that a second piece of light is reflected back into the optical fiber as well ${ }^{159}$. These two pieces of light represent two arms of an interferometer which interfere and show cantilever displacement information. 


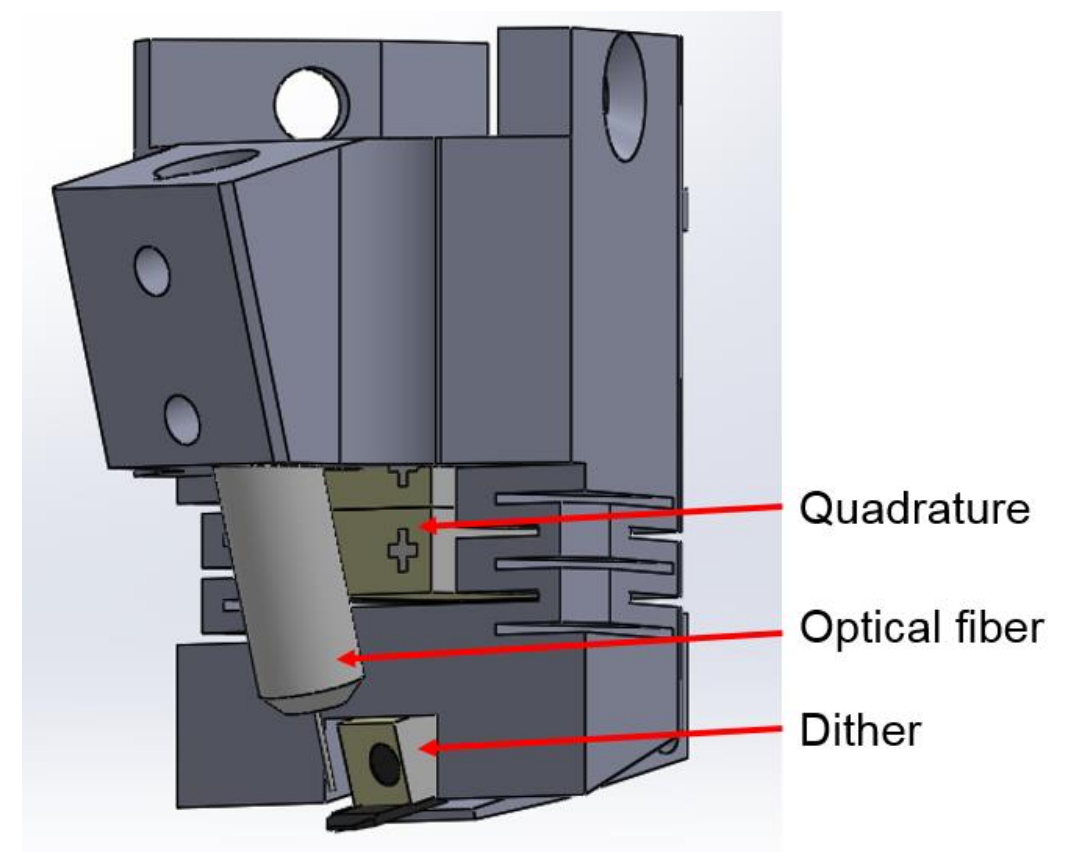

Figure 2.14 Schematic of flexure interferometer feedback system.

The interferometer arm reflected within the optical fiber has a constant phase assuming laser stability. However, the cantilever is separated from the optical fiber by the flexure body design. A piezo actuator known as the quadrature is used to stretch the flexure and change the optical path length of the second interferometer arm. As a DC voltage is applied to the quadrature, clear interference fringes are seen by the photodetector as the relative optical path length is changed (figure 2.15). The quadrature is given a set point with feedback on these fringes at a location with maximum slope. As small displacements of the cantilever take place, it results in a sensitive change to the photodetector signal. This change in signal is a direct consequence of the force applied on the cantilever tip.

A second piezo actuator known as the dither is placed in direct contact with the cantilever. This piezo actuator is responsible for the mechanical, resonant oscillation of the cantilever for noncontact mode operation. The dither piezo is strategically positioned so that the cantilever is placed directly on the piezo actuator during operation. The resonance curve of the cantilever with this experimental setup is shown in figure 2.16a. A frequency sweep is taken with a constant driving amplitude to find the greatest oscillation amplitude of the cantilever. There is one clear Gaussian peak representing the resonant frequency of the tip. In contrast, the resonance curve in figure $2.16 \mathrm{~b}$ comes from the oscillations being supplied through the metal body by the quadrature. Many other 
resonance peaks are obviously coupled into the overall signal. This is known as a "forest of peaks", and is a consequence of mechanical resonances of the metal body being excited ${ }^{160,161}$.

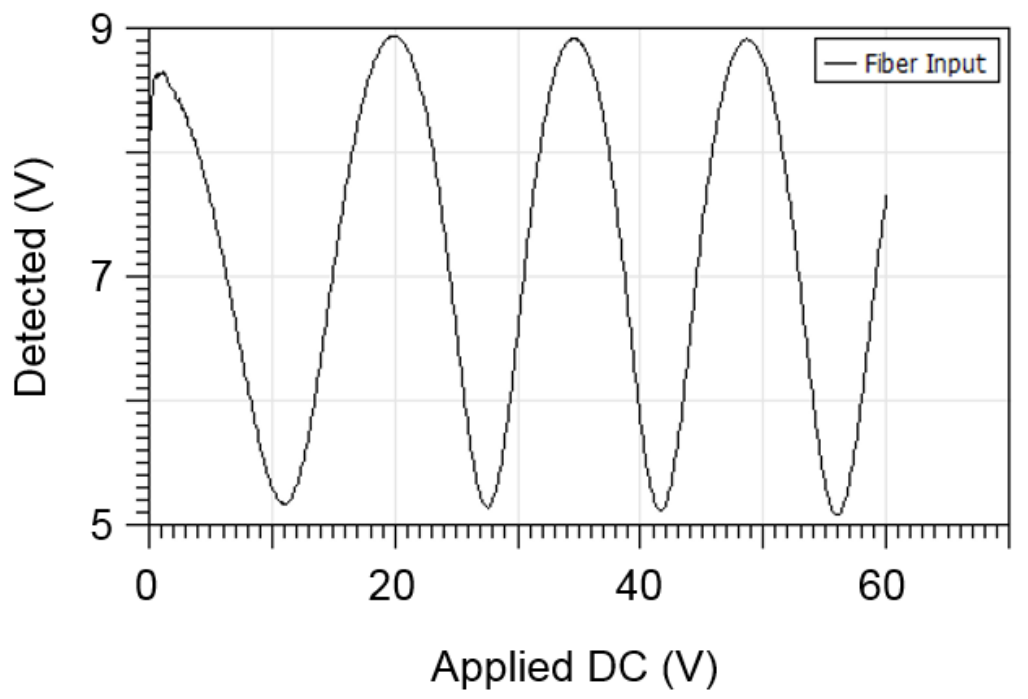

Figure 2.15 Flexure interferometer displacement measurement. As a DC voltage is applied to the quadrature piezo, its length increases and stretches the flexure body design. The difference in optical path length of the two interferometer arms is increased creating interference fringes.
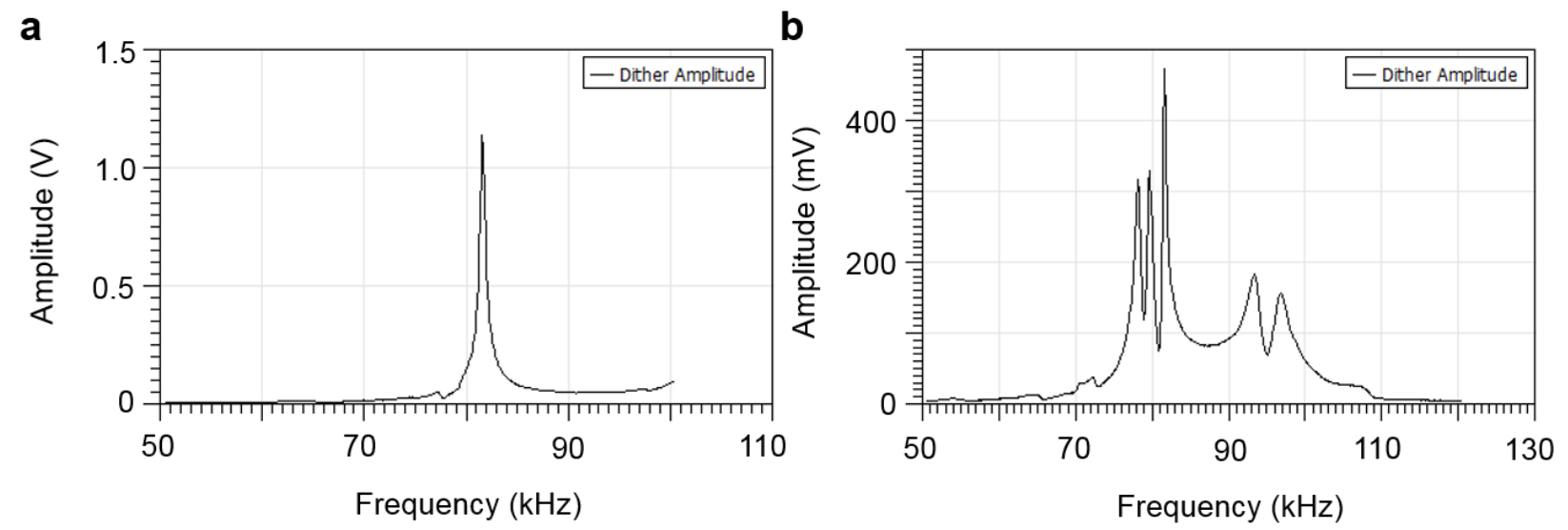

Figure 2.16 Cantilever resonance curves (a) Resoance curve when the oscillations are caused by a dither piezo in direct contact with the cantilever. (b) "Forest of peaks" created by resonances of the metal AFM body when oscillations are induced through the metal body.

By designing the home-built AFM with specific spatial constraints, it has been transformed into a SNOM. The interferometer feedback system and minimal body design has allowed a completely exposed cantilever tip and an abundance of space for optical elements. The external 
optics then induce a polarization of the AFM tip, field interaction with the sample surface, and collection of scattered light. Optical information is obtained by the scanning probe with nanoscale spatial resolution, independent of the optical wavelength. The tip is also in close proximity to the sample surface scattering near-field optical data as well as far-field. This home-built SNOM is also high vacuum, variable temperature, and has an abundance of electrical connections and lock in amplifiers. This is a versatile SNOM design that allows environmental control for a variety of experimental designs.

\subsection{In situ KPFM + SNOM}

The work done in this thesis is largely based on all of the scanning probe based methods discussed thus far in chapter 2 . As you will see in chapter 5, it became necessary to measure both KPFM and SNOM simultaneously. Correlation between the surface potential and dielectric function is used to understand $\mathrm{VO}_{2}$ phases and their stability. However, speed of measurement is an issue for understanding temporal evolution. The $\mathrm{VO}_{2} \mathrm{OVs}$ diffuse away over time, and scanning probe based images are lengthy; typically about 10 - 30 minutes. Thus taking a KPFM image and then a SNOM image takes 20-60 minutes. In that time frame much of the diffusion process has taken place, and the two images are not a direct correlation.

With that issue in mind, let's review how the different imaging modes are run. Non-contact topography measurements are based on a piezo actuated resonance of the cantilever. This takes place at the fundamental resonance frequency, $\mathrm{f}_{0}$, of the cantilever. In these experiments a $70 \mathrm{kHz}$ cantilever is used. Changes in topography effect the oscillation amplitude of the tip. Then a lock in amplifier demodulates the cantilever oscillation amplitude at $f_{0}$. FM-KPFM adds on top of that an $\mathrm{AC}$ bias at a low frequency, $\mathrm{f}_{\bmod }=5 \mathrm{kHz}$. This creates a side band frequency of the cantilever, $\mathrm{f}_{0}+\mathrm{f}_{\text {mod, }}$, which is based on the electrostatic interaction between the tip and sample. The surface potential influence on the cantilever oscillation is demodulated by another lock in amplifier at $\mathrm{f}_{0}+\mathrm{f}_{\text {mod. }}$ Both the topography and KPFM images are taken in situ. SNOM images are also taken in situ with topography. While the topography is being measured, scattered light from the tip is collected for SNOM. Another lock in amplifier is used to demodulate the scattered light at higher harmonics of the tapping frequency, 2 or $3 \mathrm{f}_{0}$. SNOM measurements do not require any new resonance of the cantilever. 

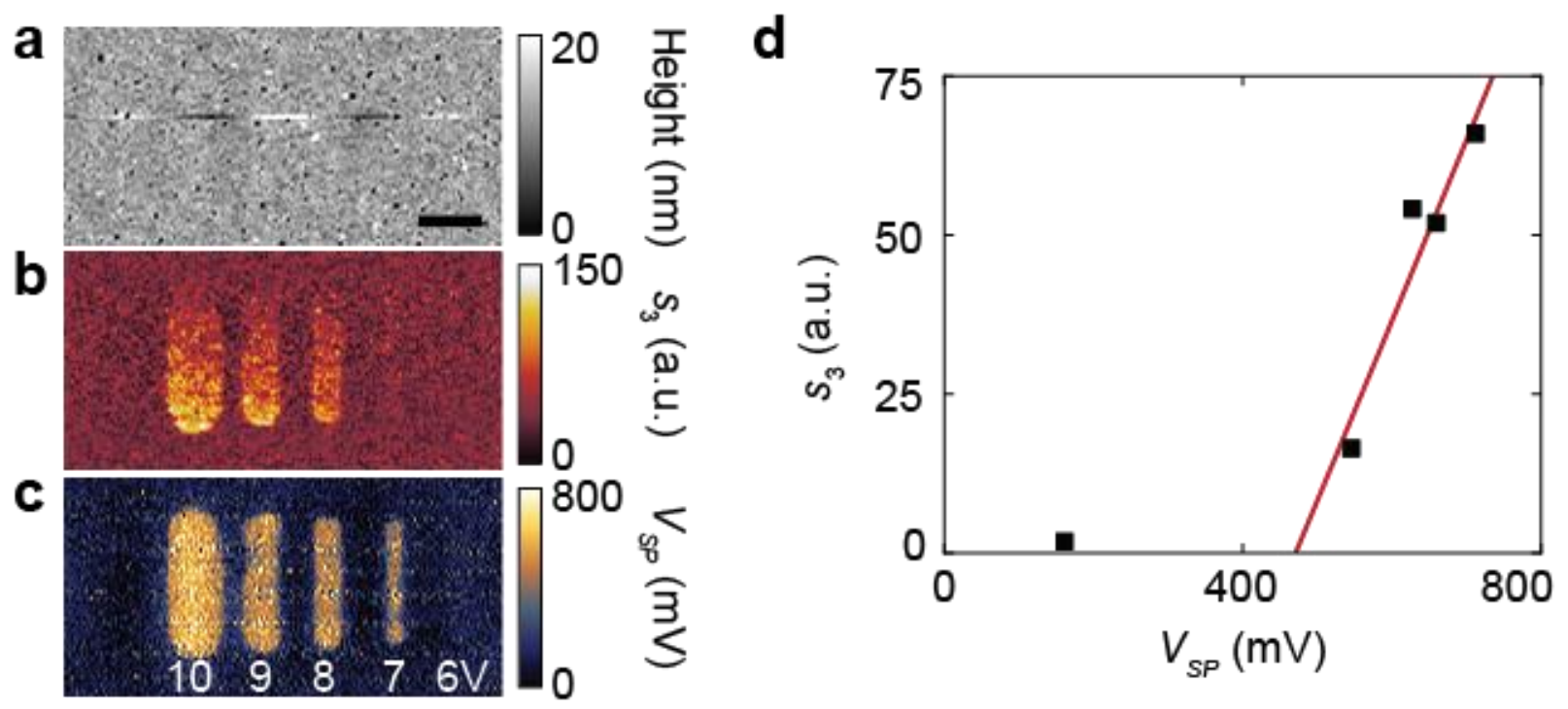

Figure 2.17 In situ SNOM and KPFM images. (a) Topography of the $\mathrm{VO}_{2}$ film surface. (b) SNOM image of a scanning probe induced metallic $\mathrm{VO}_{2}$ phase induced into the insulating $\mathrm{VO}_{2}$ background. (c) KPFM image of the $\mathrm{VO}_{2}$ phases and the voltage applied during the scanning probe lithography process. All three images were taken in situ. (d) Correlation between the KPFM and SNOM images for the metallic $\mathrm{VO}_{2}$ phase. Scale bar represents $1 \mu \mathrm{m}$.

This then brings up the question, can all three measurements be taken in situ? It has never been done before! The answer is yes, with enough lock in amplifiers. Figure 2.17 demonstrates all three images being taken in situ of a metallic $\mathrm{VO}_{2}$ phase being induced into the insulating $\mathrm{VO}_{2}$ film by scanning probe lithography. This figure demonstrates all the scanning probe functionalities that have been discussed so far. AFM non-contact topography, KPFM surface potential measurement, and SNOM optical contrast of a phase induced by scanning probe lithography are all measured in situ by the home-built SNOM. 


\section{Chapter 3}

\section{Nanoscale Phase Manipulations of $\mathrm{VO}_{2}$}

In this dissertation, one of the three major goals listed in the introduction is to induce localized phase manipulations of $\mathrm{VO}_{2}$ whereas previous works have been demonstrating macroscopic phase transitions ${ }^{162-168}$. Decreasing the domain size will allow for a higher density of binary pixels for memory elements, display screens, or even monolithic photonic devices that will be demonstrated later in chapter 4. This chapter is dedicated to demonstrating and characterizing a variety of localized $\mathrm{VO}_{2}$ phase transitions. Various scanning probe lithography methods are used for single step stimuli application with a $30 \mathrm{~nm}$ tip apex radius. Using these methods, multi-type phase transitions going beyond the regular thermodynamic phase diagram can be produced. These anomalous material phases are patterned in a reconfigurable and stable manner which allows them to be subsequently studied by systematic electrical/structural/optical characterizations.

The $\mathrm{VO}_{2}$ thin films used in all of the following experiments were grown by molecular beam epitaxy (MBE) on r-plane $\mathrm{Al}_{2} \mathrm{O}_{3}$ at Pennsylvania State University ${ }^{50}$. The films are all $30 \mathrm{~nm}$ thick. First a characterization and identification of the thermally activated IMT is taken of the thin film. In the as-grown films, a sharp transition at $T_{\mathrm{C}}=345 \mathrm{~K}$ from the room temperature $(\mathrm{RT})$ monoclinic insulating phase to the rutile metallic phase was detected (figure 3.1). On the top of figure 3.1a, the electrical resistance drops by 3 orders of magnitude across the IMT. The bottom plot is monitoring the far-field optical reflectance of the film showing the dramatic optical property modulation achieved. Temperature dependent SNOM amplitude images were also taken throughout this temperature sweep. They show the nucleation of metallic phase domains beginning to at $342 \mathrm{~K}$. Then the domains begin to grow with temperature until at $348 \mathrm{~K}$, the entire film is a uniform metallic structure. The temperatures shown in the SNOM images are also marked in the resistance and reflectivity plots by dotted lines. Note that as the metallic domains begin to take form at $342 \mathrm{~K}$, there is an immediate change to the optical reflectivity. However, the electrical resistance does not become affected until the metallic puddles begin to merge together forming constant electrical pathways. The electrical resistance does not change until about $345 \mathrm{~K}$. The surface topography remains unaffected throughout the temperature sweep. 


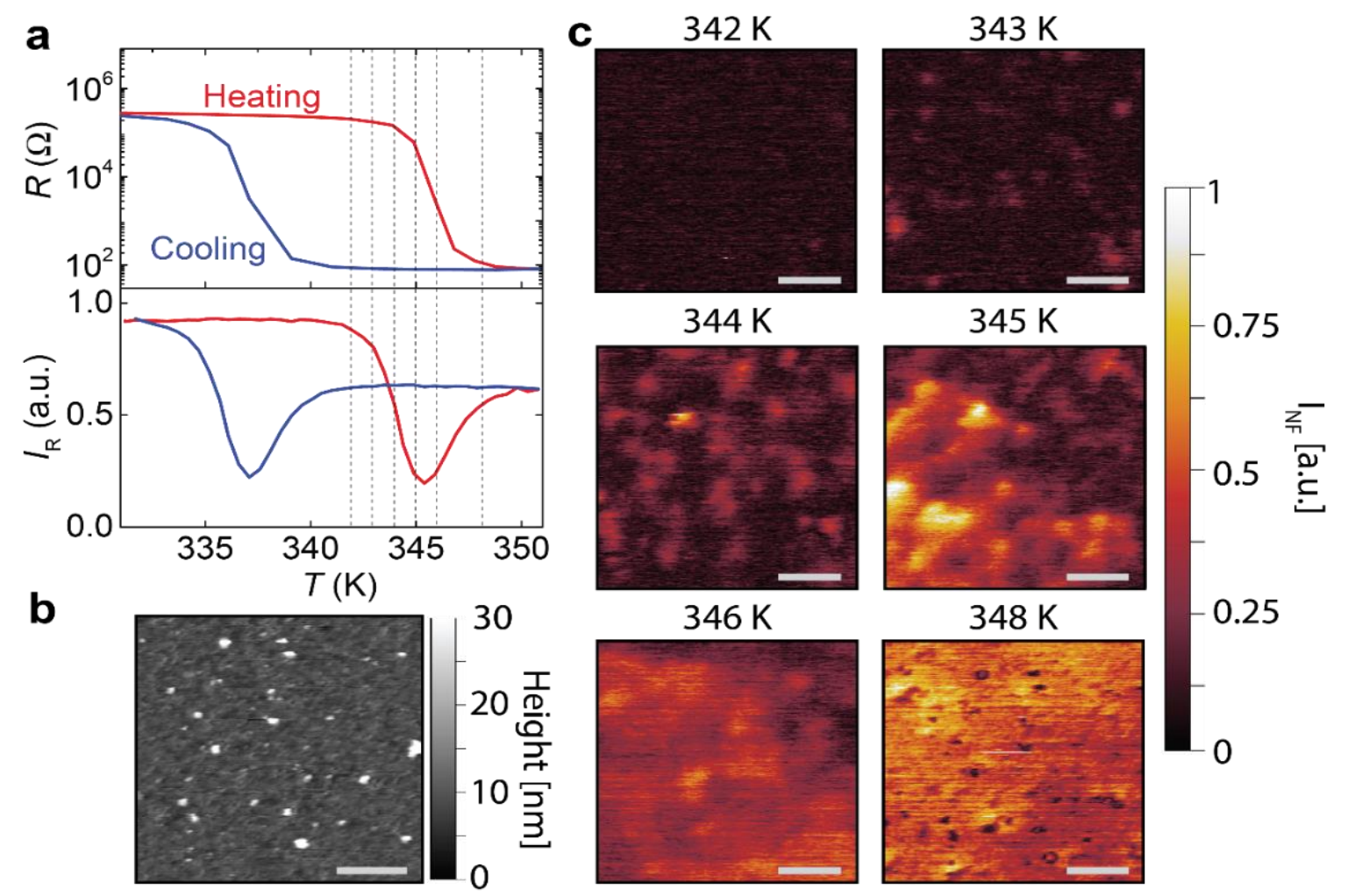

Figure 3.1 Property modulation of $\mathrm{VO}_{2}$ thin film across the IMT (a) electrical resistance (top) and $10.8 \mu \mathrm{m}$ far-field optical reflectance (bottom) vs temperature. (b) Surface topography of $\mathrm{VO}_{2}$ film remained unchanged during temperature sweeps. (c) SNOM images of the same area revealed the temperature dependent emergence and growth of metallic regions around the transition temperature. The six temperatures displayed are also labeled in (a) by dash lines. Scale bars indicate $1 \mu \mathrm{m}$.

Now I will move on to scanning probe based phase manipulations. The techniques used are summarized in figure 3.2. I start by describing the overall observations, then the detailed methodology and mechanisms of each phase are characterized and discussed in the succeeding paragraphs. From the pristine film, a monoclinic metallic phase can be created by a positively biased AFM probe (Figure 3.2b,c). For the convenience of discussion, I refer to this phase as "PB", short for positive bias. Subsequent scans on top of PB structures with negative probe bias generates a van der Waals layered compound of Vanadium Oxide hydrates (Figure 3.2d,e). I refer to this phase as "PNB", positive bias first and then negative bias. When the pristine film is maintained at a temperature near but below $T_{\mathrm{C}}$, a metastable rutile phase can be induced by the contact strain from an unbiased probe. I refer to this metallic phase as " $\mathrm{ZB}$ " for zero bias. 


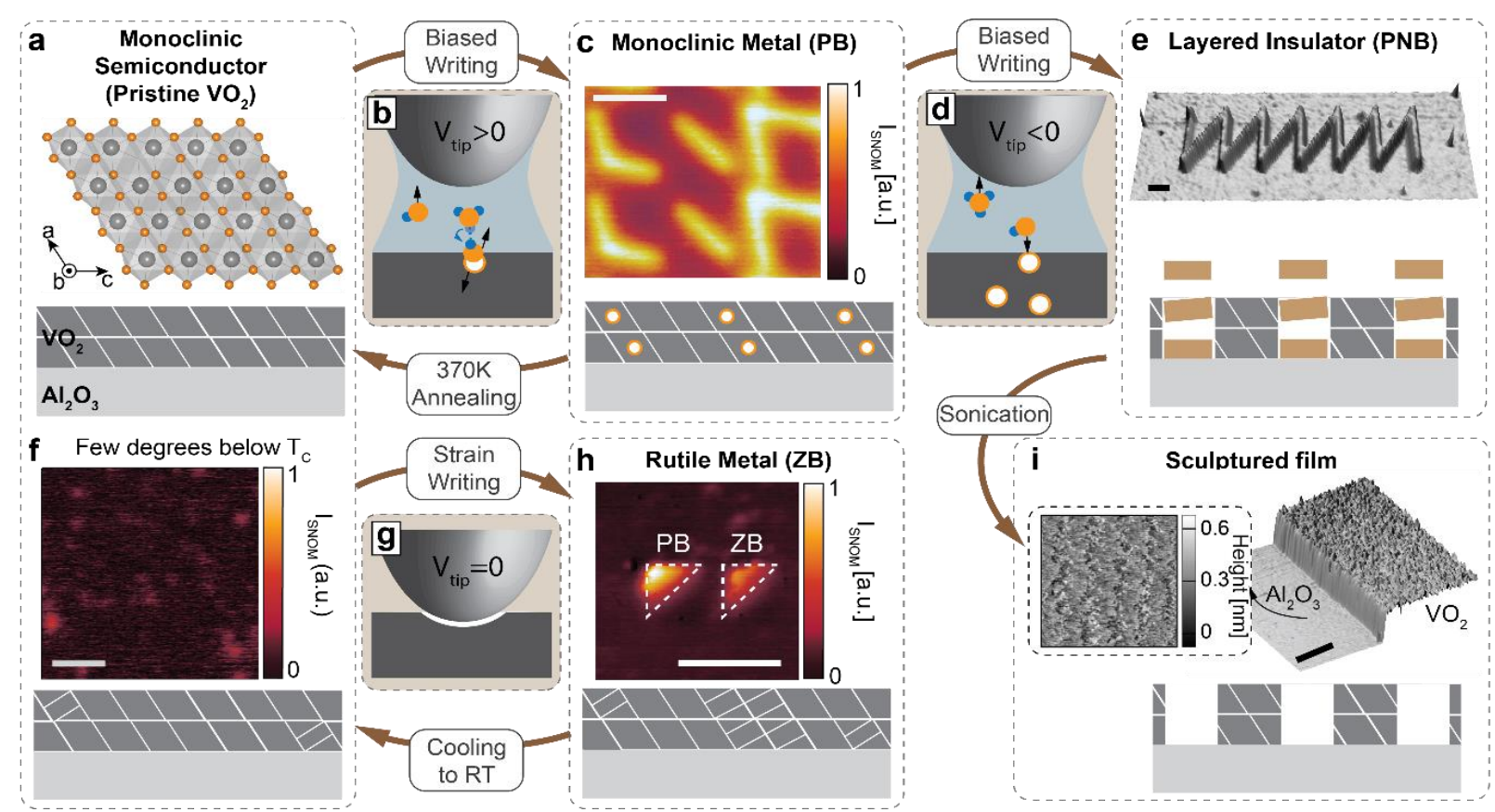

Figure 3.2 Overview of scanning probe induced phase transitions. (a) Monoclinic insulating state of $\mathrm{VO}_{2}$ film. (b) $\mathrm{PB}$ induced surface electrochemical process creates a monoclinic metallic phase. (c) SNOM image of metasurface pattern of PB structures. Annealing the sample above $T_{c}$ erases the $\mathrm{PB}$ phase, restoring pristine $\mathrm{VO}_{2}$. (d) PNB induced surface electrochemical process further transforms the PB phase into a van der Waals layered insulating compound. (e) AFM image shows $50 \%$ lattice expansion in PNB structures. (i) PNB completely removed by sonication and leads to a nano-sculptured film. AFM image shows the locally exposed $\mathrm{Al}_{2} \mathrm{O}_{3}$ terraces after sonication. (f) By heating the pristine film to a few degrees below $T_{C}$, onset of rutile phase occurs. (g) At such temperatures, contact strain can switch the local area into rutile phase. (h) SNOM image shows a strain induced $Z B$ structure and a bias induced PB structure side-by-side. $Z B$ structure restores to pristine after being cooled to RT. All scale bars indicate $1 \mu \mathrm{m}$.

The lithographic precision and advantage can be seen in figures 3.2c, e, h. Synchronized control over the probe scan trajectory, bias, and loading force allows nanoscale-microscale twodimensional patterns of $\mathrm{PB}, \mathrm{PNB}$, and $\mathrm{ZB}$ structures to be created in an on demand manner with just a single step. Then the patterns are reconfigurable as well because they can be erased and then rewritten. ZB structures restore to the pristine insulating phase after cooling the sample to RT. PB structures are stable at RT but can be erased by annealing the sample above $T_{\mathrm{C}}$. Using these 
methods, both $\mathrm{PB}$ and $\mathrm{ZB}$ structures can be repeatedly written and modified without any observable material degradation. PNB layered structures are also highly stable in ambient conditons but can only be removed completely by sonication, leaving behind a nano-sculptured film as shown in figure 3.2i.

\subsection{ZB Phase}

I will first identify and discuss the formation of the $\mathrm{ZB}$ phase. It is important to note that with MIR frequencies, small carrier density modifications usually have only negligible effects on the dielectric constant of the material. Instead, the generation of sizable SNOM contrast requires significant changes in the electronic characteristics, which makes SNOM a highly effective tool for detecting phase transitions (figure 3.1) 1694464.

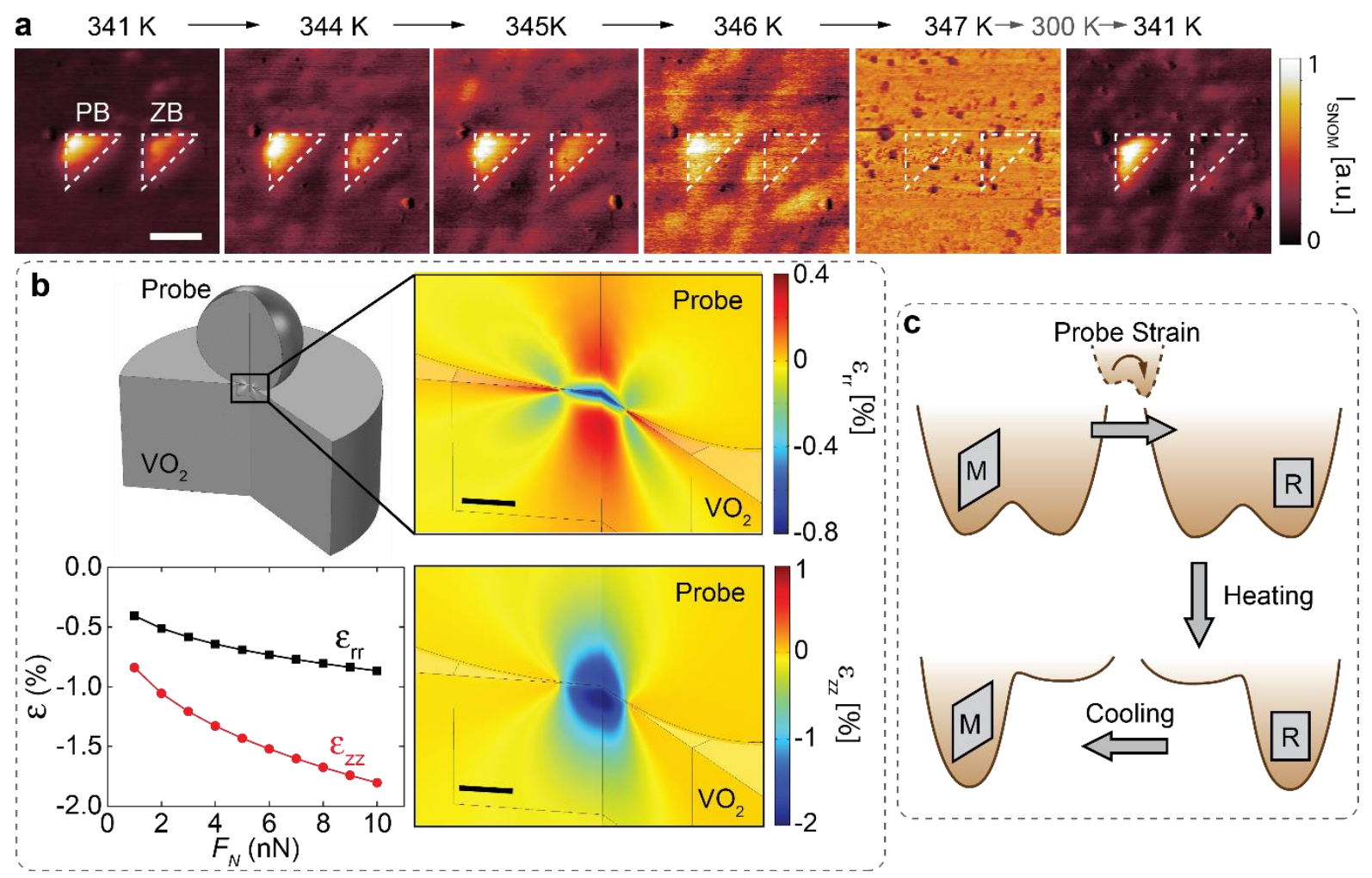

Figure 3.3 Strain induced metastable phase. (a) SNOM images taken through a temperature cycle. The two triangular metallic regions are created by positive bias $(P B)$ and zero-bias $(Z B)$ contact mode scans at $341 \mathrm{~K}$. Scale bar is $500 \mathrm{~nm}$. (b) Simulated distribution of in-plane and outof-plane strain underneath the probe and their dependences on the probe loading force. Scale bar is $2 \mathrm{~nm}$. (c) Possible mechanism of metastable phase transition by probe-induced strain. 
Figure 3.3 shows the MIR SNOM amplitude images of two triangles written side-by-side at $341 \mathrm{~K}$, just before reaching the IMT. The two triangles were written using the same probe and same loading force. In this experiment, the left-side pattern was inscribed using a positive probe bias (PB) while in contact mode which will be discussed further later, and the one on the right was written with zero probe bias $(\mathrm{ZB})$ while in contact mode. Unlike the unpatterned region in the monoclinic insulating phase, the two triangles both exhibited strong near-field intensities that are similar to the high-temperature rutile metallic phase (figure 3.1). However, after thermal cycling the sample back to RT and then up to $341 \mathrm{~K}$ again, the ZB triangle completely disappeared leaving only the PB triangle visible in the SNOM image. To reiterate, the sample was heated to the rutile phase, cooled back to RT, and then brought back to the initial $341 \mathrm{~K}$. The distinct temperature sensitivities of $\mathrm{PB}$ and $\mathrm{ZB}$ structures suggest that they likely represent two different material structures. I attribute the ZB structure to a metastable rutile phase stabilized by the probe induced contact $\operatorname{strain}^{81,86}$. Through finite element method simulation, it was found that a compressive strain as large as $1.8 \%$ can be generated in the film region underneath the probe with a loading force less than $10 \mathrm{nN}$ (figure 3.3b). Such strain level is comparable to what has been achieved in mechanically bent $\mathrm{VO}_{2}$ nanobeams where greater than a $10 \mathrm{~K}$ decrease of the IMT transition temperature was reported ${ }^{86}$. As illustrated in figure $3.3 \mathrm{c}$, when the energy barrier separating the monoclinic and rutile phases is lowered by thermal heating, probe-induced compressive strain can trigger the local formation of a lower-temperature rutile structure (ZB). Though, just like the regular high-temperature rutile phase, the $\mathrm{ZB}$ phase is energetically unfavorable at $\mathrm{RT}$, and thus will restore to the monoclinic phase after thermal cycling. Thus the ZB phase is a metastable rutile phase that can be induce locally, but still does not solve $\mathrm{VO}_{2}$ stability issues under ambient conditions. It is likely that stiffer cantilevers could be used to apply more strain to reduce the transition temperature further.

\subsection{PB \& PNB Phases}

In contrast to the $\mathrm{ZB}$ phase, the metallic $\mathrm{PB}$ phase is found to be highly stable at RT and have many interesting properties. For instance, PB structures created by a biased AFM probe have a $\sim 0.7 \mathrm{~nm}$ topographical height difference comparing to the pristine region (figure $3.4 \mathrm{a}$ ). This is a $2.3 \%$ out of plane lattice expansion which is similar to the $3 \%$ out of plane expansion found during 
field effect gating experiments performed on $\mathrm{VO}_{2}{ }^{62,63,82,99}$. Next figure $3.4 \mathrm{~b}$ shows the transient resistance change measured between two surface electrodes as a $1 \mu \mathrm{m}$ wide, $15 \mu \mathrm{m}$ long PB channel was being written by a raster-scanning probe. Although the resistance measurements were significantly shunted by the parallel conducting background of pristine $\mathrm{VO}_{2}$, steep resistance reduction steps were clearly resolved at each line scan. Conductivity of the PB channel was extracted by finite element method and found to be around 50 times larger than pristine $\mathrm{VO}_{2}$ at RT. Upon cooling to low temperatures, the conduction between electrodes connected by a PB channel remained ohmic from $300 \mathrm{~K}$ to $2 \mathrm{~K}$. The gradual resistance increase saturated below 100 $\mathrm{K}$ in figure 3.4c, indicating a metallic transport that was not restricted by band gap. This behavior is very similar to the Arrhenius plots of doped semiconductors.
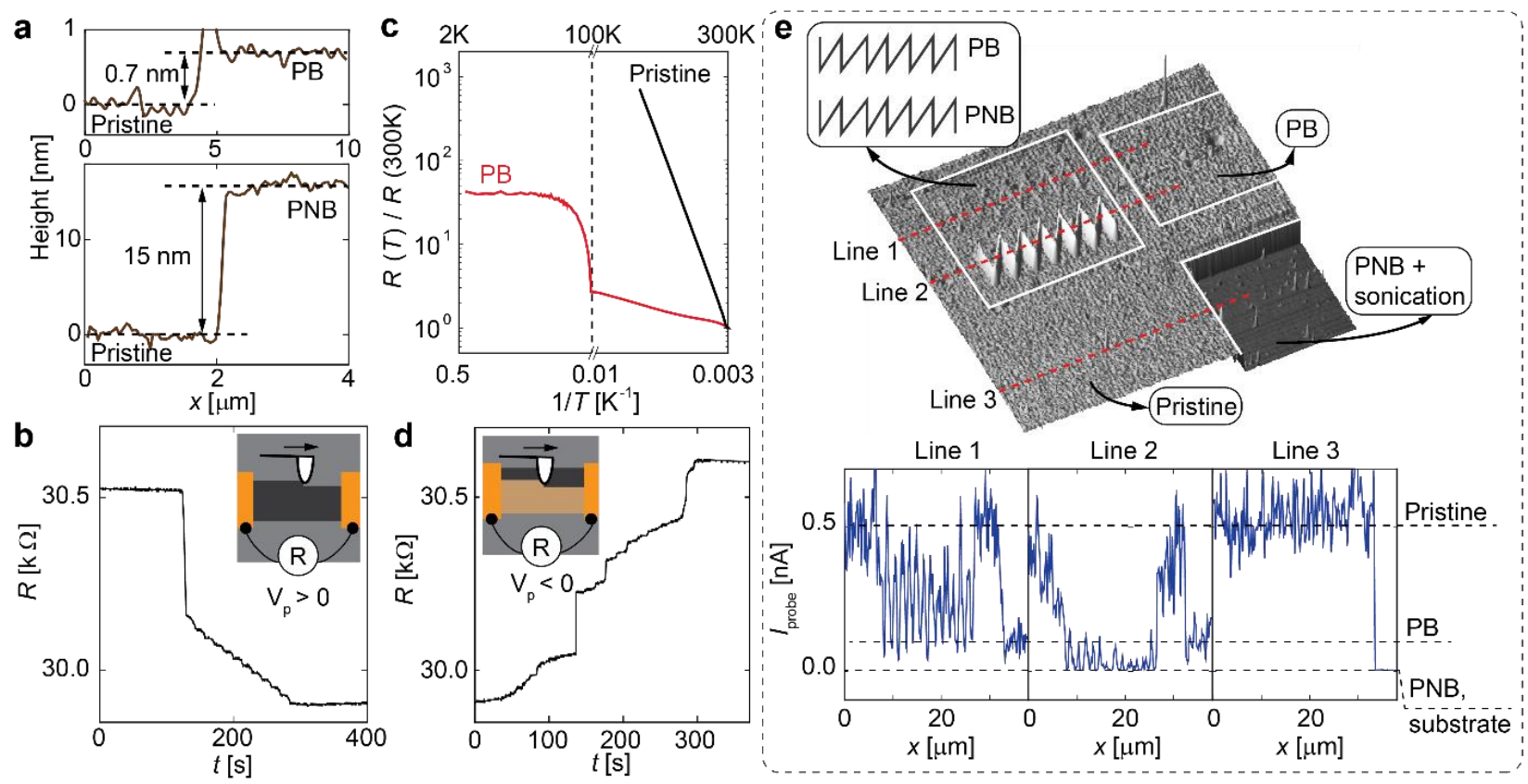

Figure 3.4 Surface topography and electrical properties of PB and PNB phases. (a) AFM topography measured at the edge of a PB region (top) and a PNB region (bottom). (b) Transient resistance changes measured when positively biased probe was raster-scanning on pristine film to produce a PB channel between two electrodes. (c) Temperature dependent resistance changes in pristine film and PB phase. (d) Transient resistance changes measured when negatively biased probe was converting the PB channel to PNB phase. (e) Topography of a region containing several different written structures (top), and the conducting AFM line profiles measured at different positions within this region. 
Besides in-plane transport, out-of-plane electrical conduction was also characterized by conducting AFM measurements in figure 3.4e. In this measurement, current flowing through the scanning probe was measured at a bias of $0.1 \mathrm{~V}$, too small to generate any observable material modifications. Interestingly, probe current was much lower in the PB regions comparing to pristine film, which seems to contradict the results of in-plane conductance measurements. I attribute this discrepancy to an insulating $\mathrm{V}_{2} \mathrm{O}_{5}$ surface layer formed in air ${ }^{170}$ that significantly affected the outof-plane conductance measurements. X-ray photoelectron spectroscopy (XPS) measurements (figure 3.5) were performed to verify this using a commercial Physics Electronics PHI 5000 Versa Probe system equipped with a focused monochromatic X-ray source (Al K $\alpha$ at $1486.6 \mathrm{eV}$ with $100 \mu \mathrm{m}$ beam size). All XPS experiments were carried out at RT at a pressure better than $10^{-8} \mathrm{mbar}$ and showed that the $\mathrm{V}^{5+}$ concentration at the surface of metallic $\mathrm{PB}$ structures was larger than the pristine film, likely caused by a thicker $\mathrm{V}_{2} \mathrm{O}_{5}$ layer.

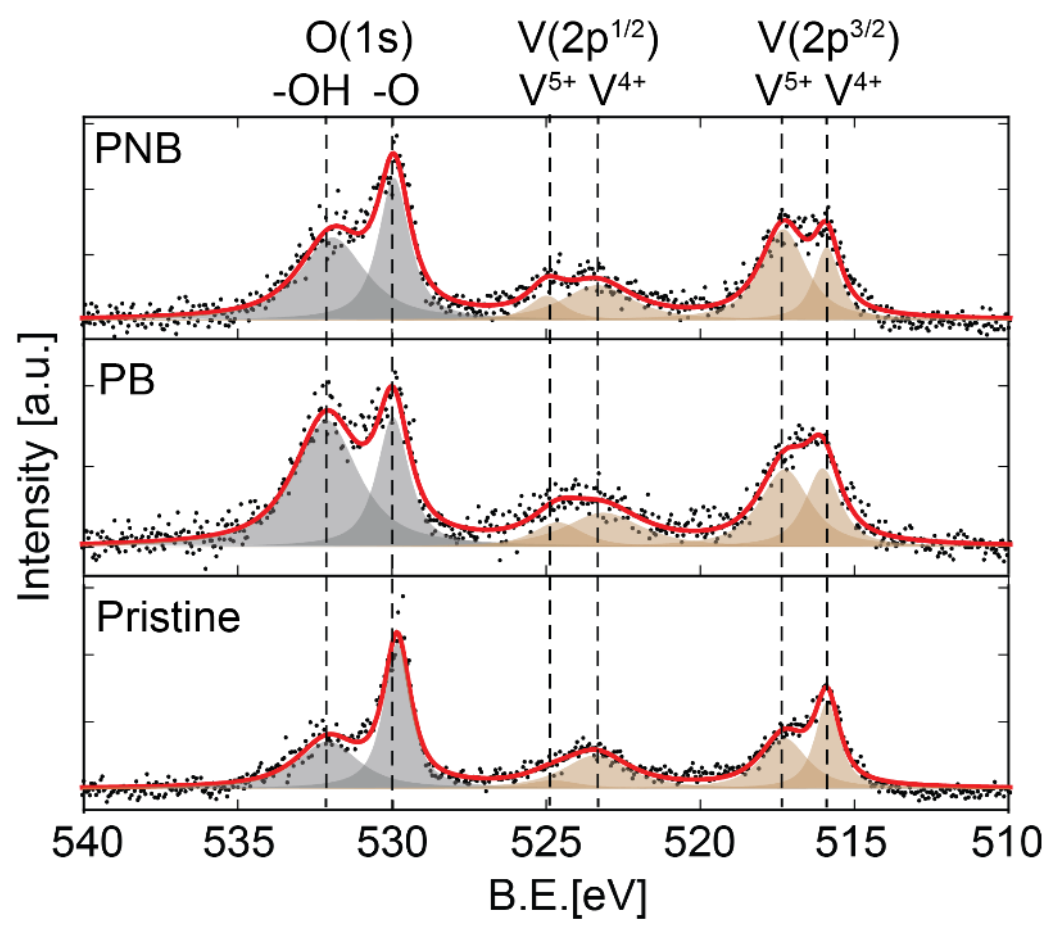

Figure 3.5 XPS spectrums measured in different $\mathrm{VO}_{2}$ phases.

Now I will compare these same properties for the PNB phase. Figure 3.4d shows the transient resistance increase measured between two surface electrodes when the PB channel was being converted to the PNB phase by a negatively biased probe on the same area. After the scan, the 
resistance became even larger than the pristine value measured before the PB channel was formed, indicating the large resistivity of the PNB phase. Zero probe current was found when conducting AFM measurement was performed over the PNB regions (figure 3.4e), further supporting its insulating character. The most interesting property is the topography. The surface of written PNB structures rose above the $30 \mathrm{~nm}$-thick pristine film by $15 \mathrm{~nm}$ (figure 3.4a), corresponding to a large volume expansion of $50 \%$. The in plane dimensions of PNB structures can be easily controlled by probe bias amplitude and scan speed as expected in scanning probe lithography, but the large out of plane expansion ratio was very robust and barely affected by writing parameters (figure 3.6).
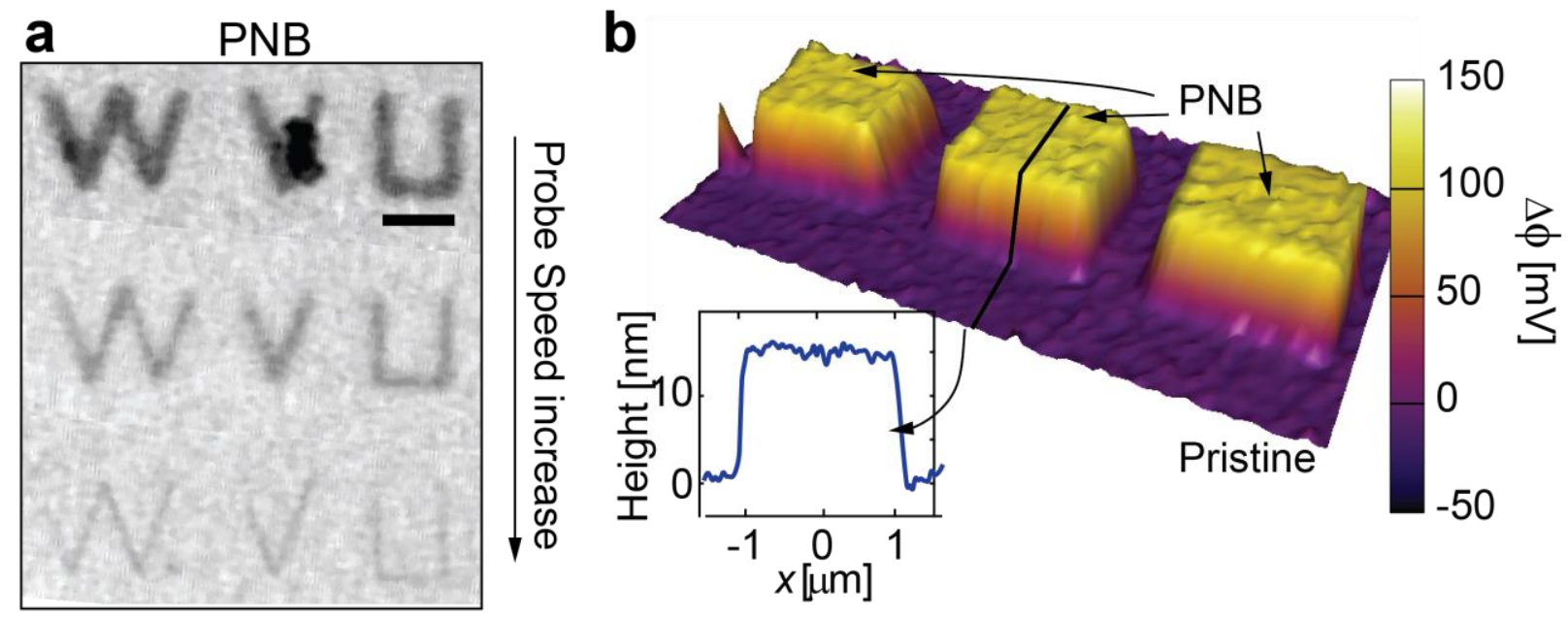

Figure 3.6 Images of PNB structures with different writing speeds. (a) Optical image of patterns written with probe speeds of $50 \mathrm{~nm} / \mathrm{s}, 100 \mathrm{~nm} / \mathrm{s}$, and $200 \mathrm{~nm} / \mathrm{s}$. Scale bar indicates $5 \mu \mathrm{m}$. (b) Work functions measured by KPFM overlaid on their topography. The three squares were written with different probe speeds of $200 \mathrm{~nm} / \mathrm{s}, 100 \mathrm{~nm} / \mathrm{s}$ and $50 \mathrm{~nm} / \mathrm{s}$ (from left to right), but exhibited the same height increase. The inset shows the topography line profile across one square.

One more question remaining to characterize these phases is to try and identify the lattice structures. Micro-X-ray diffraction ( $\mu$ XRD) (figure 3.7) and electron backscatter diffraction (EBSD) (figure 3.8) measurements were performed to try and provide this information. $\mu$ XRD was performed using a monochromatic $11 \mathrm{keV}$ x-ray beam with sub-micron resolution at the 7-ID-C beamline of the Advanced Photon Source ${ }^{171}$. The x-ray beam was focused down to a spot of radius of $\sim 500 \mathrm{~nm}$ by a Fresnel zone plate, which is large enough to avoid sample damage and is 
sufficiently small to provide necessary spatial resolution for the patterned region. EBSD data was acquired on an FEI Quanta 600 scanning electron microscope using the Oxford NordlysMax ${ }^{2}$ EBSD system. Acquisition of data was performed at $10 \mathrm{kV}$ and a beam current on the order of hundreds of pAs. Monoclinic $\mathrm{VO}_{2}$, rutile $\mathrm{VO}_{2}$, and $\mathrm{Al}_{2} \mathrm{O}_{3}$ phases were selected from the existing phase databases for indexing Kikuchi bands.

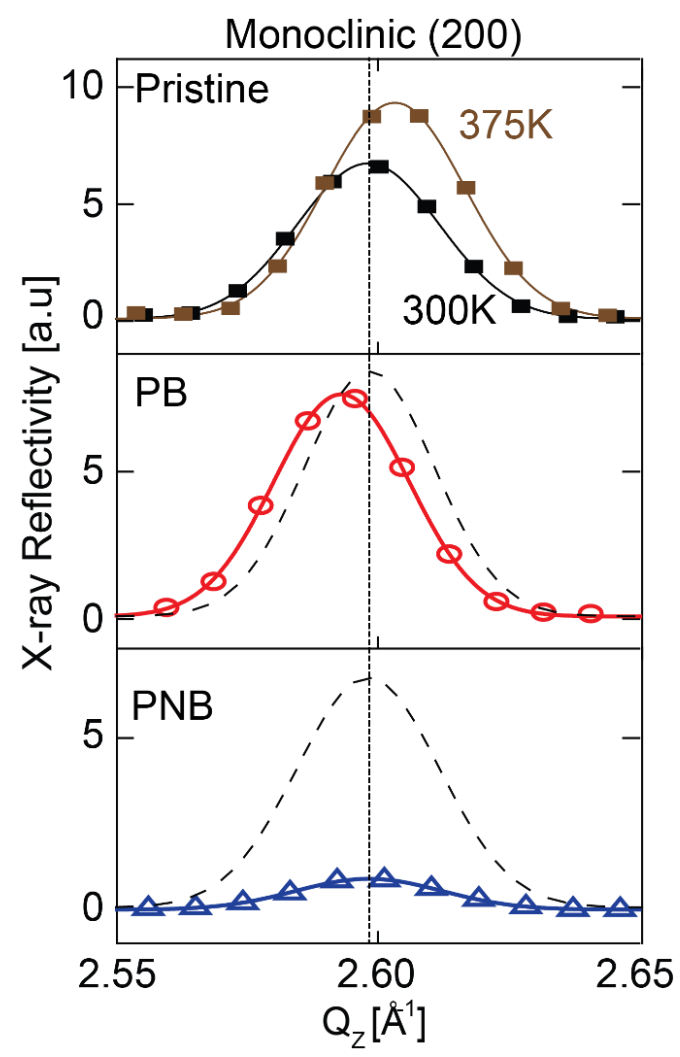

Figure 3.7 Structural properties by $\mu X R D$ spectrums of phases near the monoclinic (200) peak.

In the pristine region, the monoclinic (200) Bragg peak $\left((200)_{\mathrm{M}}\right)$ was observed in $\mu \mathrm{XRD}$. Also EBSD showed clear Kikuchi patterns of the monoclinic structure. At temperatures above $T_{C}$, $\mu \mathrm{XRD}$ showed the $(200)_{\mathrm{M}}$ peak shifted to higher out-of-plane scattering vector $\left(Q_{Z}\right)$, consistent with the reduced lattice spacing along the c-axis in the rutile phase ${ }^{172}$. In the PB region, the $(200)_{M}$ peak position was shifted to a lower $Q_{Z}$ value compared to the pristine film in figure 3.7 which agrees with the lattice expansion seen by AFM. The EBSD pattern in figure 3.8 then observed the same Kikuchi pattern as pristine $\mathrm{VO}_{2}$. These observations indicate that the PB region has a 
monoclinic crystalline structure. In contrast, diffraction intensities of X-ray and backscattered electrons were both very weak in the PNB region, indicating the loss of long range crystalline order. It is likely that the PNB phase has an amorphous characteristic similar to when $\mathrm{VO}_{2}$ undergoes ion bombardment ${ }^{64,173}$.
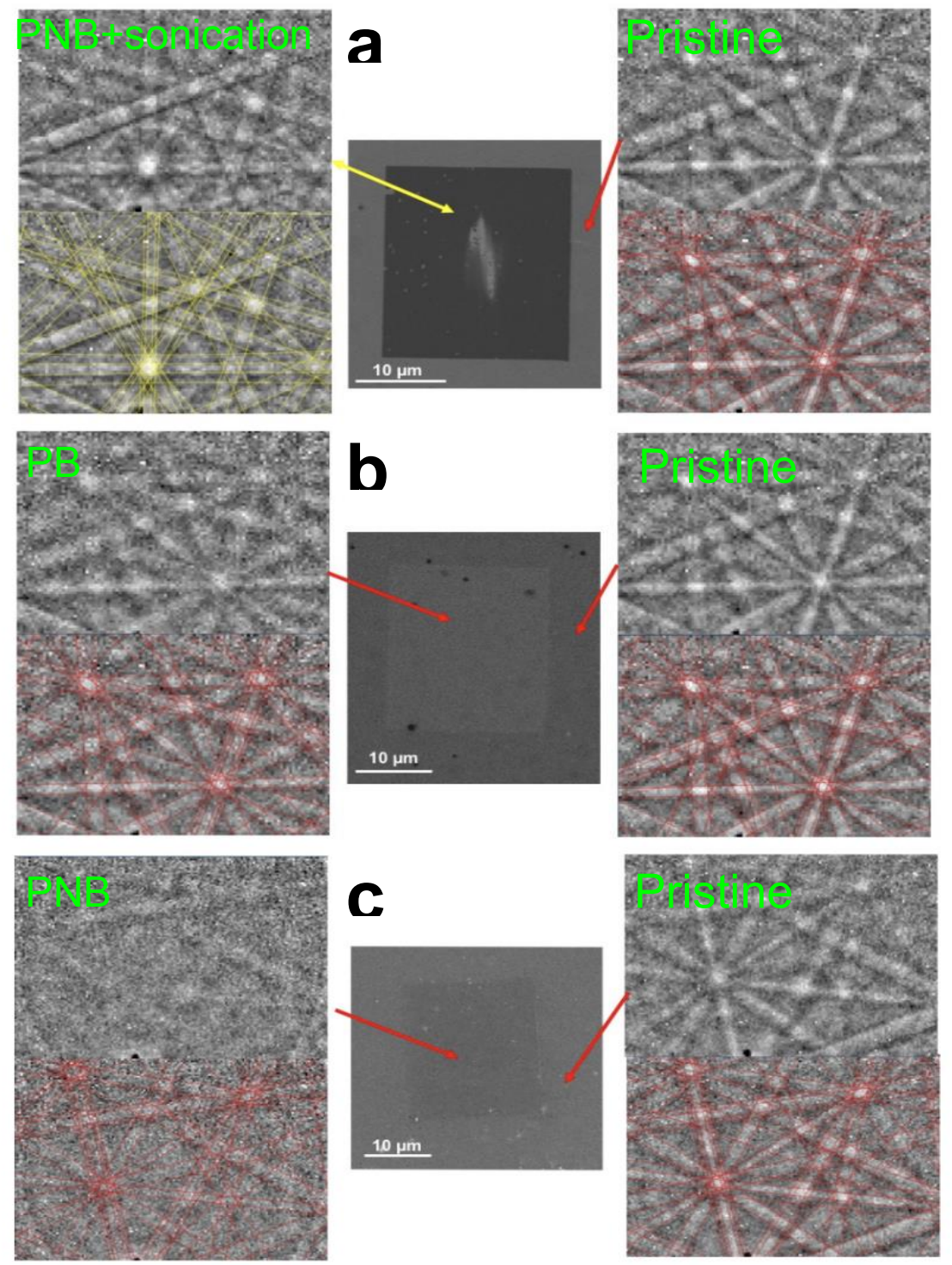

Figure 3.8 EBSD patterns of different $\mathrm{VO}_{2}$ phases obtained on (left) and off (right) different written square structures with the indexed Kikuchi bands shown below. Middle column shows the SEM images of the written structure. Yellow indexed phase is hexagonal $\mathrm{Al}_{2} \mathrm{O}_{3}$, while the red phase is monoclinic $\mathrm{VO}_{2}$. 
Under typical ambient conditions with 40\%-45\% relative humidity at $293 \mathrm{~K}$, PB structures are reliably written. They just require contact mode scans at $1 \mu \mathrm{m} / \mathrm{s}$ and tip biases larger than $10 \mathrm{~V}$. Faster writing speed can be achieved by increasing the bias magnitude. For example, under typical ambient conditions, to create a PB dot with $200 \mathrm{~nm}$ diameter requires a stationary probe dwell time of $600 \mathrm{~ms}$ at a bias of $20 \mathrm{~V}$. The minimum dwell time reduces to $200 \mathrm{~ms}$ for a bias of $30 \mathrm{~V}$ and $150 \mathrm{~ms}$ for a bias of $40 \mathrm{~V}$.

To understand the formation mechanism of the PB phase, control experiments with varied bias conditions and writing sequences were performed in figure 3.9. PB channels (connecting electrodes 3,4 and 5,6 as marked in figure 9a) were written by applying a $20 \mathrm{~V}$ bias to the probe through a $1 \mathrm{G} \Omega$ current limiting resistor $\left(\mathrm{R}_{0}\right)$. A probe current of $2 \mathrm{nA}$ was found during the contact mode scans indicating a large contact resistance $\left(R_{C}\right)$ of $\sim 9 G \Omega$. Since $R_{C}$ is much larger than $\mathrm{R}_{0}$, the voltage across the probe-sample junction was close to the full $20 \mathrm{~V}$ and the field underneath the probe is large (figure 3.9b). For the channel between electrodes 1 and 2, the same $20 \mathrm{~V}$ bias was applied but the probe current was kept at $20 \mathrm{nA}$. This is accomplished by increasing the probe loading force to generate a significantly lower contact resistance. While the force required to form a low-resistance contact varies from tip to tip, such contact can typically be produced within reasonable number of attempts by monitoring the probe current while adjusting the AFM set point and tip approaching speed in the meantime. Since in this case the contact resistance was much less than the current limiting resistor, the increased probe current was obtained at the expense of reducing the field underneath the probe (figure $3.9 \mathrm{~b}$ ).

Unlike regular PB structures, the center channel written with large current but small field was barely visible under the optical microscope (figure 3.9a). Its electrical property also remained semiconducting, albeit with a reduced activation energy compared to the pristine film as indicated by the slope of the Arrhenius plot in figure 3.9c. Positive probe bias writing was also tested in a high vacuum of $10^{-5}$ mbar. In vacuum, the contact resistance at probe-sample junction was always very small regardless of the loading force, and no writing effects could be detected by either optical or electrical methods. Clearly, a large electric field applied across the probe-sample junction, rather than a large probe current, is the key element enabling the PB phase formation. 

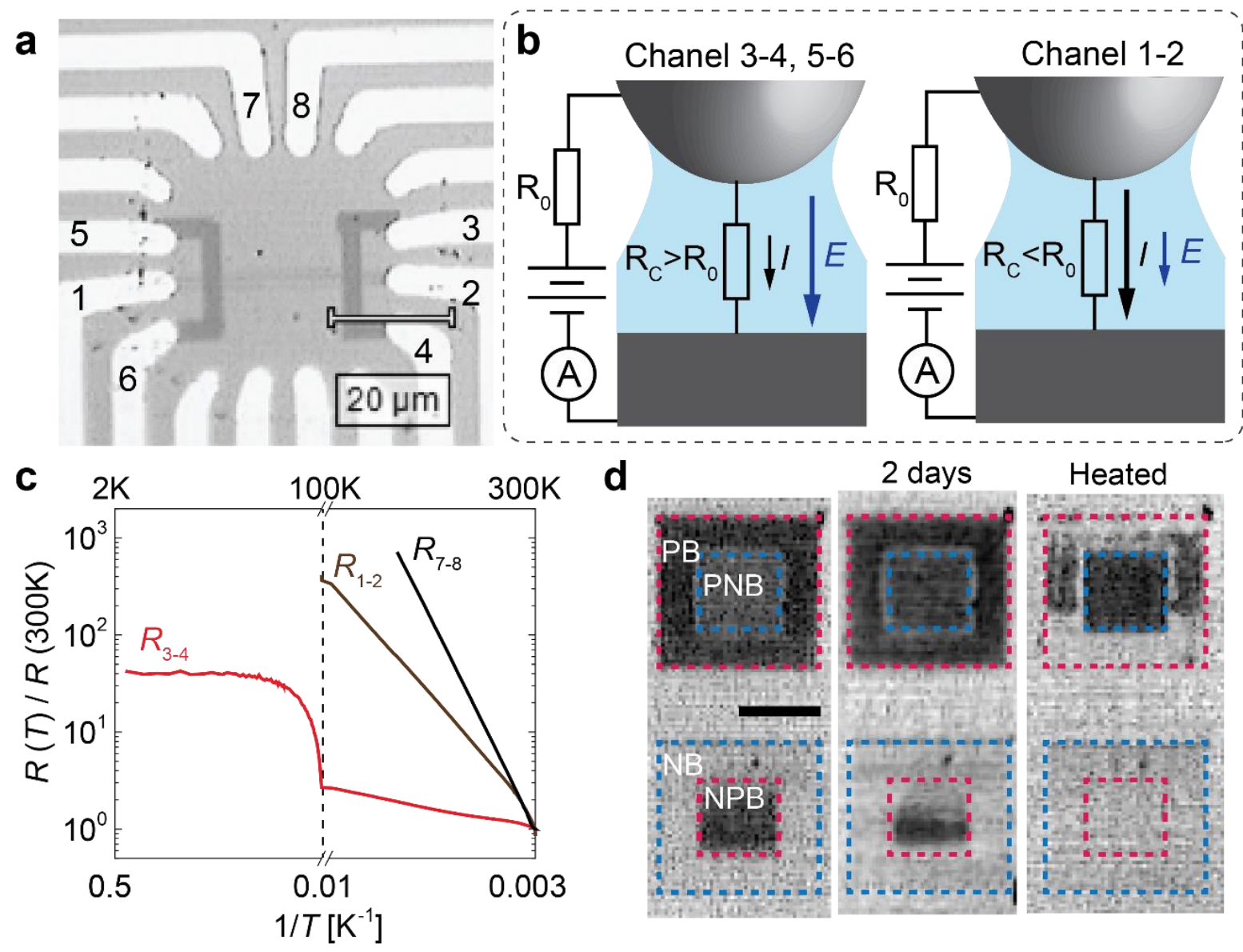

Heated

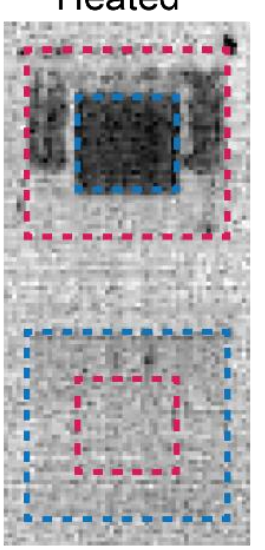

Figure 3.9 Varied writing sequences and conditions. (a) Optical image of three channels written with same probe bias but different contact resistances. (b) Influences of contact resistance on the probe-sample junction current and field. (c) Temperature dependent resistance changes measured between different pairs of electrodes. (d) Optical images of structures produced by two different writing sequences, and after they are stored in air for two days and then heated to $370 \mathrm{~K}$ for half an hour. Region enclosed by red dashed lines were scanned by positive probe bias, and regions enclosed by blue dashed lines were scanned by negative probe bias. Scale bar indicates $5 \mu \mathrm{m}$.

I propose the following mechanism to explain the formation of the $\mathrm{PB}$ phase. The $\mathrm{VO}_{2}$ film surface is hydrophilic based on the water droplet contact angle in figure $3.10^{174}$. Thus a surface water layer is formed at ambient lab conditions with a relative humidity of about $40 \%{ }^{175-178}$. Across the water meniscus formed between the AFM probe and the sample surface, electric fields larger than $10^{9} \mathrm{~V} / \mathrm{m}$ can be generated by small probe biases ${ }^{178-182}$. Under such intensive fields, ionization of water molecules $\left(2 \mathrm{H}_{2} \mathrm{O} \rightarrow \mathrm{H}_{3} \mathrm{O}^{+}+\mathrm{OH}^{-}\right)$occurs at a high rate ${ }^{179183184}$, and the water meniscus acts like a nanoscale "electrolyte" puddle (figure 3.2b,d). At positive probe biases, reducing $\mathrm{H}_{3} \mathrm{O}^{+}$ 
ions are driven toward the sample surface by the field and react with the oxygen ions provided by $\mathrm{VO}_{2}$, leaving OVs behind in the film similar to electrolyte gating experiments discussed in chapter 1. Similar tip bias induced electrochemical reactions ${ }^{183-189}$ have also been reported in other materials including metals, semiconductors, and oxides, where localized oxidation/reduction can be generated depending on the nature of the sample material and the bias polarity applied. Charge neutrality of the defect formation mechanism leaves the oxygen-bond electrons in the lattice, which occupy the conduction band formed from vanadium $d$ orbitals ${ }^{62,82,88,190}$ giving rise to sufficient free carrier concentration when the local OV density becomes large enough. This explains the increased conduction. The generation of large densities of OVs is also consistent with the lattice expansion ${ }^{63,191}$ observed by $\mu \mathrm{XRD}$ and the degraded EBSD pattern as shown in figures 3.7 and 3.8 .

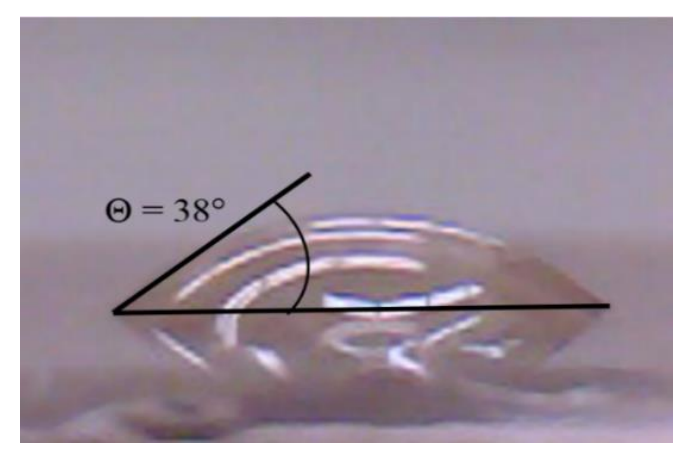

Figure 3.10 Hydrophilic water contact angle image

The generation of the PNB phase by negative probe biases can only be achieved by scanning over a PB region. Figure 3.9d shows the optical images of structures produced by two different probe scanning sequences. In the first sequence a $10 \mu \mathrm{m}$ square was first scanned with a probe bias $\left(V_{p}\right)$ of $20 \mathrm{~V}$, and then the center $5 \mu \mathrm{m}$ square was scanned for a second time with $V_{p}=-20 \mathrm{~V}$. This sequence generated a PNB square surrounded by a PB frame. In the second sequence, the first scan over the $10 \mu \mathrm{m}$ square was done with $V_{p}=-20 \mathrm{~V}$ and the second scan over the center $5 \mu \mathrm{m}$ square was done with $V_{p}=20 \mathrm{~V}$, which produced two additional region types of NB (negative $V_{p}$ only) and NPB (negative $V_{p}$ first and then positive $V_{p}$ ). Comparing to the pristine film, $\mathrm{PB}$, PNB, and NPB regions all showed clear optical contrasts, while the NB region was visibly unchanged, indicating that directly applying negative bias to the pristine film has much less effect. Under negative probe bias, strongly oxidative hydroxyl ions $\left(\mathrm{OH}^{-}\right)$from ionized water are driven 
toward the film. However, likely due to the difficulty for large hydroxyl ions to migrate deeper into the crystalline lattice, these ions had little effect on pristine $\mathrm{VO}_{2}$ film when writing with NB. Whereas in $\mathrm{PB}$ regions with a higher density of oxygen vacancies, $\mathrm{OH}^{-}$can more easily enter the film and occupy OV sites. During this process, I hypothesize that hydrate structures may form, such as $\mathrm{VO}_{2} \cdot \mathrm{H}_{2} \mathrm{O}$ and $\mathrm{V}_{2} \mathrm{O}_{5} \cdot \mathrm{H}_{2} \mathrm{O}$ (Table 3.1). These compounds have $\mathrm{Van}$ der Waals layered structures with inter-layer distance much larger than $\mathrm{VO}_{2}$, which are consistent with the observed large volume expansion and easy material removal by sonication. The drastic crystal structure modifications associated with hydroxylation also explains the loss of long range crystalline lattice order as observed by $\mu$ XRD and EBSD in figures 3.7 and 3.8.

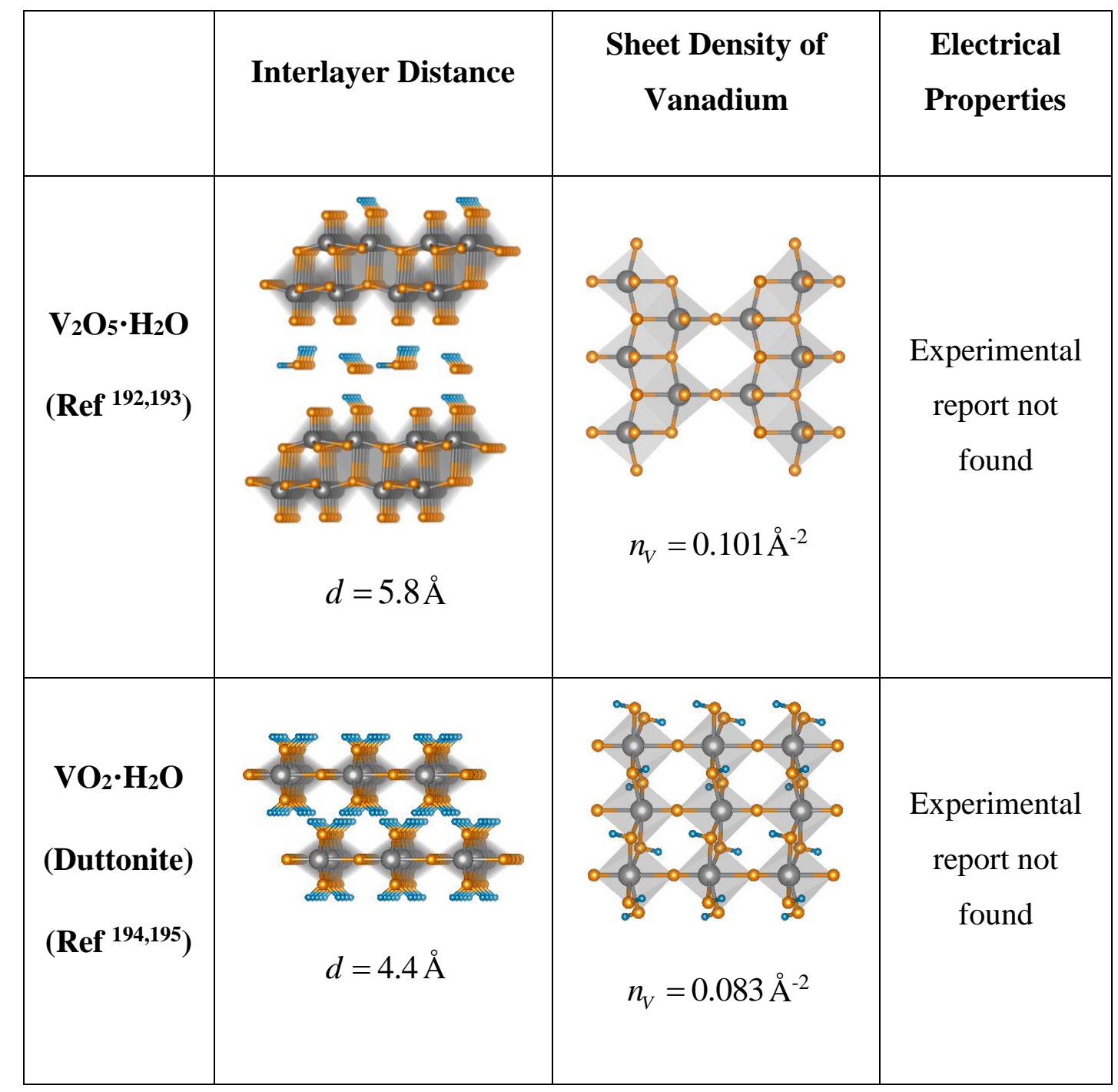

Table 3.1 Possible compounds in PNB structures. 
$\mathrm{PB}$ and PNB regions are both stable at RT in air (figure 3.9d, figure 3.11). After heating the sample to $370 \mathrm{~K}$ for half an hour to activate the rutile metallic phase, most of the PB structure transitioned back to the pristine state. A complete reversal was achieved by prolonging the heating step to an hour. This reversion was not only shown in optical reflectance but also verified by Raman spectroscopy (figure 3.11). In contrast, the layered compounds in PNB phase remained stable after being heated, and are only removed by sonication.

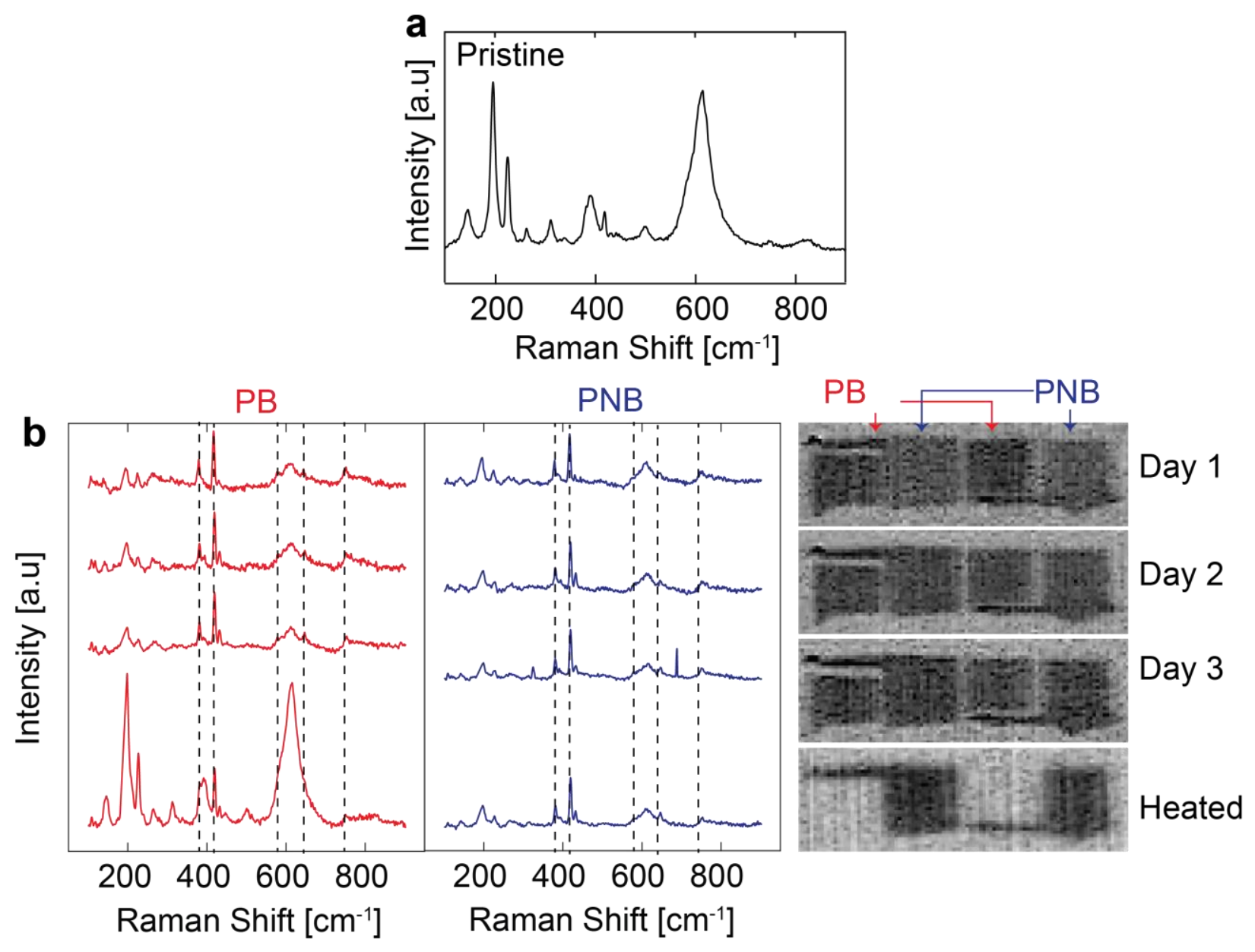

Figure 3.11 Raman spectra of different $\mathrm{VO}_{2}$ phases (a) Raman spectrum of pristine monoclinic $\mathrm{VO}_{2}$. (b) Evolution of Raman spectrum and optical contrast in PB and PNB phases in air at room temperature and after heating to $370 \mathrm{~K}$ for an hour. Small but sharp Raman peaks marked by dashed lines are from the $\mathrm{Al}_{2} \mathrm{O}_{3}$ substrate. The rest of the peaks are from the monoclicnic phase while the rutile phase is Raman inactive in this frequency range. 
To summarize, a metastable transition between monoclinic and rutile phases can be triggered by probe-induced strain. Compressive strain is known to decrease the IMT of $\mathrm{VO}_{2}$, and here for the first time localized domains of the IMT due to strain are demonstrated. Although this phase transition is not stable at RT. However, with a firmer tip to increase the amount of strain the transition temperature could be decreased even further. Also a stable metallic phase can be created at the nanoscale by performing scanning probe lithography with a positively biased AFM probe. This phase can either be restored to the regular insulating monoclinic phase by annealing the sample or further transformed into an insulating layered compound with a subsequent negative probe bias. The introduction of this dissertation argued that $\mathrm{VO}_{2}$ had three limitations (size, stability, ambient creation) for further innovation, and two out of the three have been answered and solved in this chapter. $\mathrm{VO}_{2}$ insulating to metallic phase manipulations can be controlled at ambient conditions on a localized nanoscale of about $150 \mathrm{~nm}$. 


\section{Chapter 4}

\section{Monoclinic Metallic $\mathrm{VO}_{2}$ Optical Properties}

In the previous chapter 3 , multiple localized $\mathrm{VO}_{2}$ phase transitions were demonstrated. Throughout the remainder of this dissertation, the focus will be on the monoclinic metallic phase induced by a positively biased AFM tip because this is the only localized phase transition that is metallic and stable in ambient conditions. Moving forward, I will refer to it as the MM-phase for monoclinic metallic instead of the previously used PB phase. Similarly the thermally activated rutile metallic phase will be known as the RM-phase, and the as-grown monoclinic insulating phase known as the MI-phase.

At this point the MM-phase lattice structure is known, the conductivity increase, the topographical lattice expansion, and that it is seemingly stable. Optically all I know is that there is a clear visible contrast between the MM and MI-phase. Chapter 4 will be dedicated to further understanding the optical properties of the MM-phase. Also can these properties be used for device application? The high-temperature RM phase has been shown to support surface plasmon polaritons (SPP) at infrared frequencies 41,42,44,196, coherent collective electron oscillations. However, since the thermally-driven phase transition is volatile and difficult to control at nanoscales, the studies have been very limited ${ }^{38,64,197,198}$. It is possible that the increase in carrier density due to OV doping gives the MM-phase similar SPP resonances. SNOM images in chapter 3 of the MM and RM-phases do appear very similar so their optical properties are likely similar.

\subsection{Optical \& Plasmonic Properties}

To probe for SPPs, rectangular MM-phase strips were written near the edge of a large gold electrode deposited at the sample surface as depicted in figure 4.1a. Then figure 4.1b shows a SNOM image of a $30 \mu \mathrm{m} \times 4 \mu \mathrm{m}$ MM-phase strip taken at $\lambda_{0}=2 \pi / k_{0}=10.9 \mu \mathrm{m}$. Besides of an overall material contrast between the MM-phase strip and the surrounding MI-phase region, clear fringes are also found along the strip, consistent with the propagation of SPP waves. SNOM is commonly utilized to image SPP interference fringes ${ }^{199-201}$. The saturated color portion of the SNOM image is the gold electrode, known to have a very strong SNOM signal. 

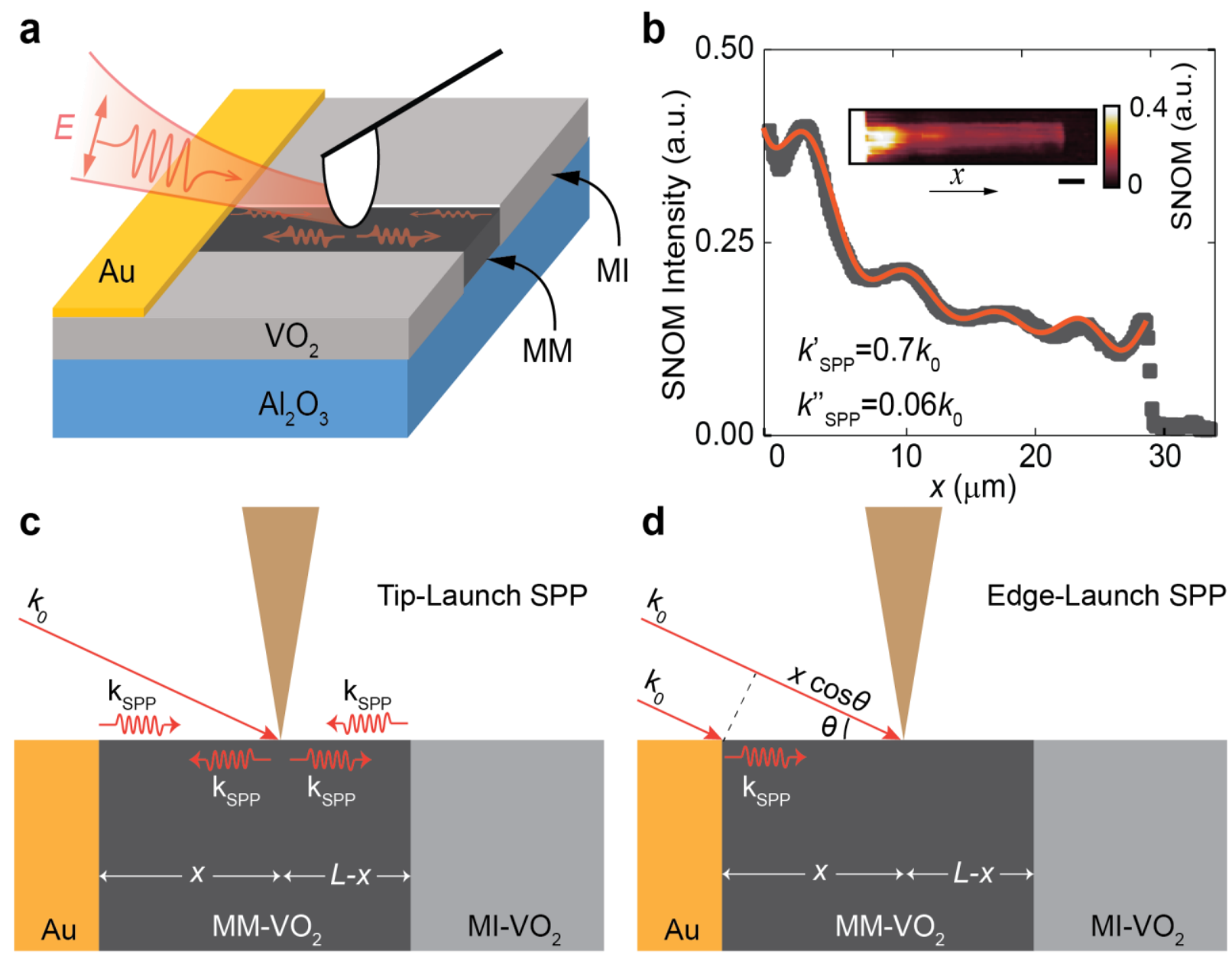

Figure 4.1 SPP propagations in MM-phase $\mathrm{VO}_{2}$ (a) Illustration of the sample configuration with the AFM-written MM-phase strip near an Au electrode. (b) Inset shows the SNOM image taken on a $30 \mu \mathrm{m} \times 4 \mu \mathrm{m}$ MM-phase strip using an incident wavelength of $\lambda_{0}=2 \pi / k_{0}=10.9 \mu \mathrm{m}$. Scale bar represents $5 \mu \mathrm{m}$. Grey dots plot the SNOM line profile along the strip. Red curve shows the fitting result based on equation 4.2. (c) Scenario 1, two opposite-traveling SPP waves are launched by the SNOM tip and reflected at the Au/MM-VO edge and the $M M-V O_{2} / M I-V O_{2}$ edge. (b) Scenario 2, SPP is launched at the Au/MM-VO edge and travels along the $\mathrm{MM}-\mathrm{VO}_{2}$ strip.

Two possible SPP launching and propagation scenarios ${ }^{199,200}$ were considered to understand these SPPs as depicted in figure 4.1c,d. In the first scenario (figure 4.1c), two SPP waves traveling in opposite directions are launched by the tip of the SNOM probe. The sharp AFM tip has been known to be the scattering source for light to launch SPPs in other experimental situations on graphene and noble metals ${ }^{199-201}$. The launched SPPs travel along the MM-phase $\mathrm{VO}_{2}$ strip and 
are reflected at the two opposing ends of the MM-phase strip. The reflected SPP waves recombine at the probe tip and are scattered into the far-field to be detected. In this case, the total electric field relative to the SNOM measurements are the superposition of the scattered fields of the two opposite-traveling SPP waves launched by the probe tip and the reference beam used in SNOM detection:

$$
\begin{aligned}
E= & E_{S P P}^{L}+E_{S P P}^{R}+E_{r e f} \\
= & A_{t i p} \exp \left[i \tilde{k}_{S P P} x\right] \tilde{r}_{M M / A u} \exp \left[i \tilde{k}_{S P P} x\right] \\
& +A_{t i p} \exp \left[i \tilde{k}_{S P P}(L-x)\right] \tilde{r}_{M M / M I} \exp \left[i \tilde{k}_{S P P}(L-x)\right] \\
& +A_{r e f} \exp [i \phi]
\end{aligned}
$$

The phase of the incident light at the tip position is used as the phase reference, $L=30 \mu \mathrm{m}$ is the length of the MM-phase $\mathrm{VO}_{2}$ strip, $\tilde{r}_{M M / A u}$ is the SPP reflectance at the MM-VO$/ A u$ boundary, $\tilde{r}_{M M / M I}$ is the SPP reflectance at the MM-VO $/ \mathrm{MI}-\mathrm{VO}_{2}$ boundary, and $\phi$ is the relative phase of the reference light that was tunable in experiments. Out of the three terms in equation 4.1, only the first two terms are modulated by the probe dithering but the strength of the last term is much stronger. Therefore, the leading terms of the lock-in detected SNOM intensity in this scenario can be expressed as:

$$
\begin{aligned}
& I_{S N O M} \sim\left|E_{S P P}\right|^{2} \\
&=I_{0}+2 A_{t i p} A_{r e f} r_{M M / A u} \exp \left[-2 k_{S P P}^{\prime \prime} x\right] \cos \left[2 k_{S P P}^{\prime} x-\phi_{1}\right] \\
&+2 A_{t i p} A_{r e f} r_{M M / M I} \exp \left[-2 k_{S P P}^{\prime \prime}(L-x)\right] \cos \left[2 k_{S P P}^{\prime}(L-x)-\phi_{2}\right]
\end{aligned}
$$

Here, $\phi_{1}, \phi_{2}$ include the phase difference between $\tilde{r}_{M M / A u}$ and $\tilde{r}_{M M / M I}$. It can be seen that, $I_{S N O M}$ in this scenario is expected to exhibit interference fringes with a width of $\pi / k_{S P P}^{\prime}$ as the sample scans (i.e. $x$ varies). Since the experimentally observed fringe width was $7.75 \mu \mathrm{m}$ (figure $4.1 \mathrm{~b}$ ), this entails that the SPP wavelength is $\lambda_{S P P}=2 \pi / k_{S P P}^{\prime}=15.5 \mu \mathrm{m}$, and $k_{S P P}^{\prime}=0.7 k_{0}$, where $k_{0}$ is the free-space wavevector of the incident light.

In the second scenario (figure 4.1d), the SPP wave is launched at the gold edge which then travels rightward toward the SNOM probe, also a well-documented scenario ${ }^{199-201}$. The total field in this case is: 


$$
\begin{aligned}
E & =E_{S P P}^{A u / M M}+E_{r e f} \\
& =\exp \left[-k_{0} x \cos \theta\right] A_{A u / M M} \exp \left[i \tilde{k}_{S P P} x\right]+A_{r e f} \exp [i \phi]
\end{aligned}
$$

, where $\theta=25^{\circ}$ is the light incidence angle. Following equation 4.3, the corresponding SNOM intensity becomes:

$$
\begin{aligned}
I_{S N O M} & \sim\left|E_{S P P}\right|^{2} \\
& =I_{0}+2 A_{A u / M M} A_{r e f} \exp \left[-k_{S P P}^{\prime \prime} x\right] \cos \left[\left(k_{S P P}^{\prime}-\cos (\theta) k_{0}\right) x-\phi\right]
\end{aligned}
$$

, which is expected to exhibit a fringe width of $2 \pi /\left(k_{S P P}^{\prime}-\cos (\theta) k_{0}\right)$. Again, considering the experimental fringe width of $w=7.75 \mu \mathrm{m}$, the SPP wavelength in this case should be $\lambda_{S P P}=2 \pi / k_{S P P}^{\prime}=\frac{2 \pi}{2 \pi / w+\cos (\theta) k_{0}}=4.7 \mu \mathrm{m}$, and $k_{S P P}^{\prime}=2.4 k_{0}$.

Fittings according to both equation 4.2 and 4.4 were performed to the SNOM data shown in figure $4.1 \mathrm{~b}$ to find the corresponding $k_{S P P}^{\prime \prime}$ values. The results based on equation 4.2 were $k_{S P P}^{\prime}=0.7 k_{0}, k_{S P P}^{\prime \prime}=0.06 k_{0}$, and results based on equation 4.4 were $k_{S P P}^{\prime}=2.4 k_{0}, k_{S P P}^{\prime \prime}=0.12 k_{0}$. While the best agreement with experimental data was achieved using equation 4.2, to more accurately identify the origin of the SPP mode, I further examined the two fitting results by comparing them to the physical model that describes the SPP propagation in the dielectric/metal/dielectric tri-layer structure. In such structures, when the metal layer is thick, two decoupled SPP modes are present, one bounded by the top metal/dielectric interface and another by the bottom interface. When the metal film thickness decreases below the optical penetration depth $(\sim 200 \mathrm{~nm})$, as is the case in the air/30 $\mathrm{nm} \mathrm{MM-} \mathrm{VO}_{2} / \mathrm{Al}_{2} \mathrm{O}_{3}$ system studied here, the two bulk metal/dielectric SPP modes strongly hybridize and evolve into two new thin-film modes (SPP1 and SPP2). Dispersion relations of the two thin-film SPP modes can be found by solving the following equations ${ }^{202,203}$ :

$$
\begin{aligned}
& \tilde{k}_{i}^{2}=\tilde{k}_{S P P}^{2}-k_{0}^{2} \tilde{\varepsilon}_{i}, i=1\left(\mathrm{MM}-\mathrm{VO}_{2}\right), 2(\text { air }), 3\left(\mathrm{Al}_{2} \mathrm{O}_{3}\right) \\
& \exp \left(-2 \tilde{k}_{1} d\right)=\frac{\tilde{k}_{1} / \tilde{\varepsilon}_{1}+\tilde{k}_{2} / \tilde{\varepsilon}_{2}}{\tilde{k}_{1} / \tilde{\varepsilon}_{1}-\tilde{k}_{2} / \tilde{\varepsilon}_{2} / \tilde{\varepsilon}_{1}+\tilde{k}_{3} / \tilde{\varepsilon}_{3}} \cdot \frac{\tilde{k}_{1} / \tilde{\varepsilon}_{1}-\tilde{k}_{3} / \tilde{\varepsilon}_{3}}{}
\end{aligned}
$$


, where $d=30 \mathrm{~nm}$ is the thickness of the metallic $\mathrm{MM}-\mathrm{VO}_{2}$ layer, $\tilde{\varepsilon}_{2}=1$ is the air permittivity, $\tilde{\varepsilon}_{3}=0.15+i 0.05$ is the permittivity of $\mathrm{Al}_{2} \mathrm{O}_{3}$ at the MIR incidence frequency, and $\tilde{\varepsilon}_{1}=\varepsilon_{1}^{\prime}+i \varepsilon_{1}^{\prime \prime}$ is the unknown complex permittivity of MM-phase $\mathrm{VO}_{2}$.
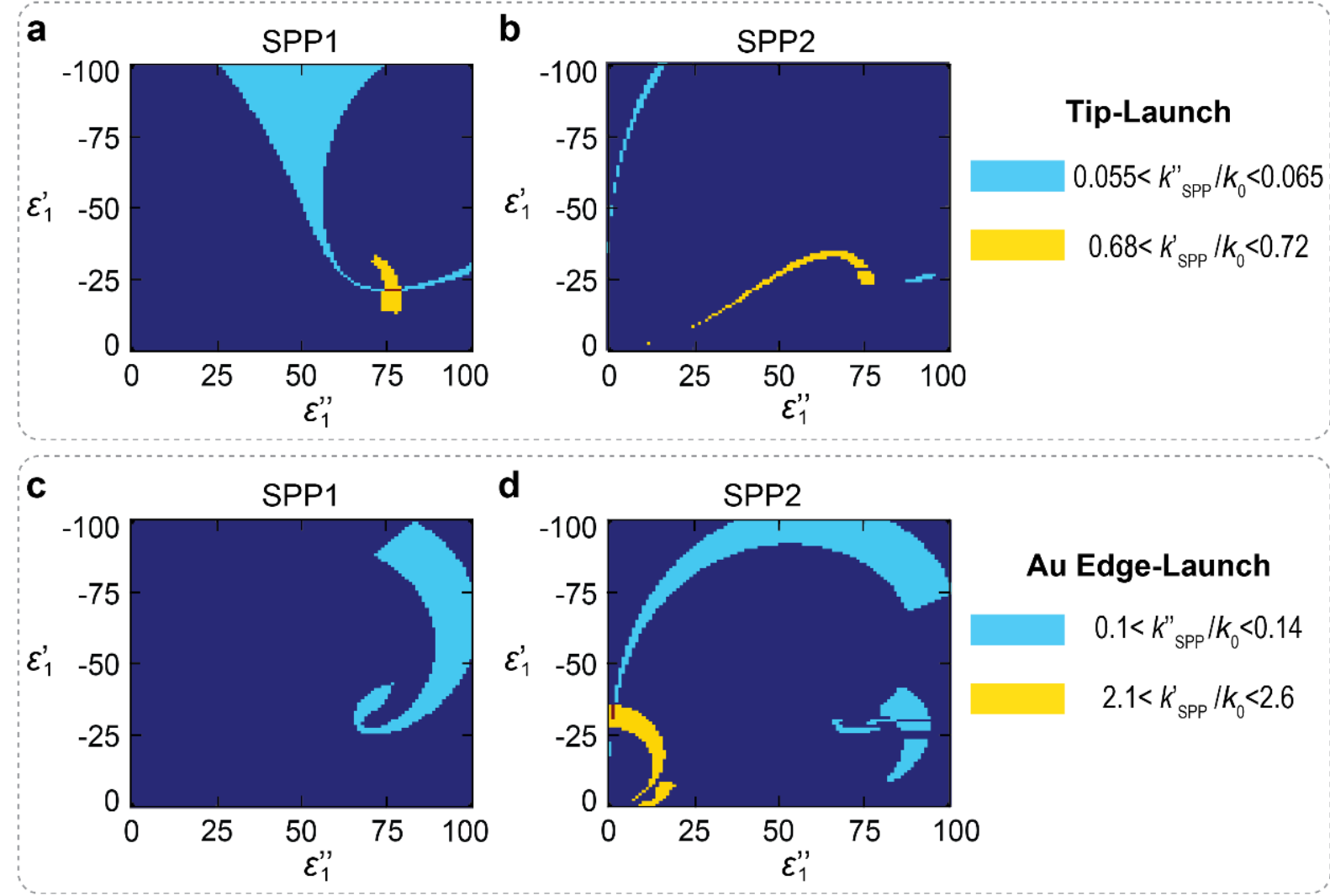

Figure 4.2 Maps of the complex permittivity matching observed SPP mode. The four maps represent four different combinations of the two thin-film SPP solutions obtained from equation 4.5 and the two SPP launching scenarios as depicted in figure 4.1.

Solving equation 4.5, I mapped out the SPP wavevectors $\tilde{k}_{S P P}=k_{S P P}^{\prime}+i k_{S P P}^{\prime \prime}$ in a large complex permittivity range $\left(\varepsilon_{1}^{\prime} \in[-100,0], \varepsilon_{1}^{\prime \prime} \in[0,100]\right)$. The results are plotted in figure 4.2 , in which the yellow areas indicate the $\left(\varepsilon_{1}^{\prime}, \varepsilon_{1}^{\prime \prime}\right)$ value region showing good match with the experimentally extracted $k_{S P P}^{\prime}$ value, and the light blue areas indicate the $\left(\varepsilon_{1}^{\prime}, \varepsilon_{1}^{\prime \prime}\right)$ value region with good agreement with the experimentally extracted $k_{S P P}^{\prime \prime}$ value. The intersection of these two types of regions mark a possible $\tilde{\varepsilon}_{1}$ value that could produce the experimentally observed SNOM signal in 
figure 4.1b. Considering both of the two solutions of equation 4.5 and the two SPP launching geometries as shown in figure $4.1 \mathrm{c}, \mathrm{d}$, there are a total four scenarios that need to be evaluated. As shown in figure 4.2, two possible solutions were found: one is $\tilde{\varepsilon}_{1}=-21+i 76$ that is associated with the tip-launched SPP1 mode, and another is $\tilde{\varepsilon}_{1}=-30+i 1.5$ which is associated with the Au edge-launched SPP2 mode.

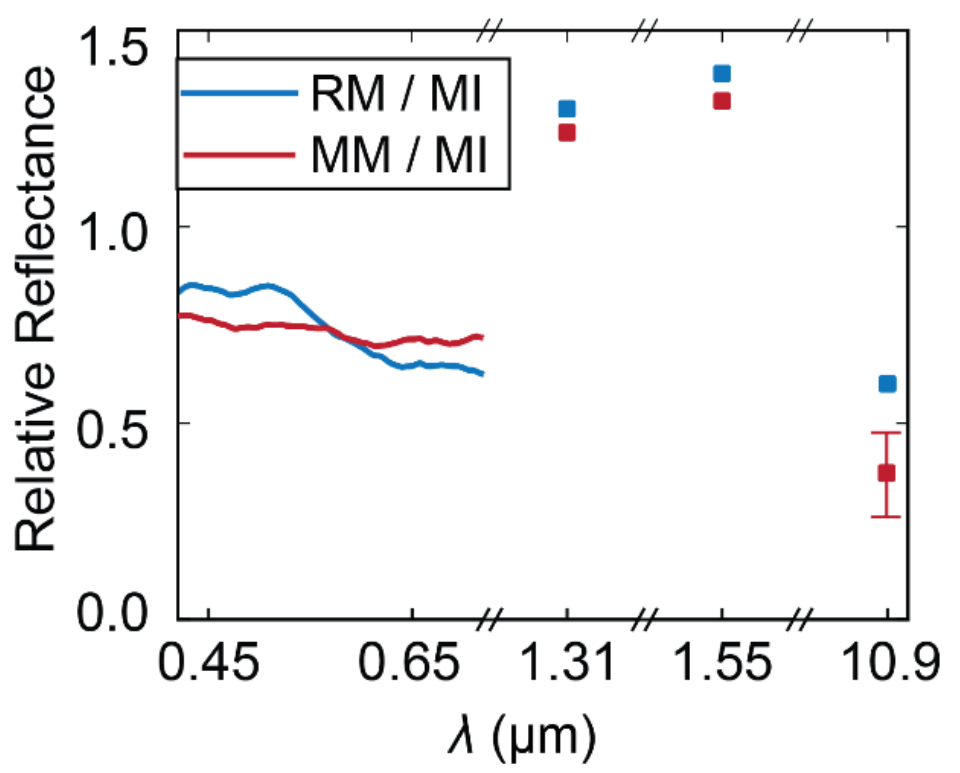

Figure 4.3 Relative reflectance comparing metallic $\mathrm{VO}_{2}$ phases. Blue curve and dots represent the measured reflectance ratio between high-temperature RM-phase and the MI-phase at various wavelengths. Red curve and dots represent the experimentally measured ratio between AFM written MM and MI-phase. Error bar for the MM-phase at $10.9 \mu \mathrm{m}$ is due to thickness variation of the film. Thickness sensitivity of reflectance at the MIR frequency can be seen in figure 4.6d.

\begin{tabular}{|c|c|c|}
\hline & $\varepsilon^{\prime}$ & $\varepsilon^{\prime \prime}$ \\
\hline$M I$ & 5.4 & 0.3 \\
\hline$M M$ & -21 & 76 \\
\hline$R M$ & -21 & 89 \\
\hline
\end{tabular}

Table 4.1 MIR (10.9 $\mu \mathrm{m})$ complex permittivity of $\mathrm{VO}_{2}$ phases 
$R=\left|\frac{r_{21}+r_{13} \exp \left[i 2 k_{0} \tilde{n}_{1} d\right]}{1+r_{21} r_{13} \exp \left[i 2 k_{0} \tilde{n}_{1} d\right]}\right|^{2}$

, where $r_{21}=\frac{\tilde{n}_{2}-\tilde{n}_{1}}{\tilde{n}_{2}+\tilde{n}_{1}}, r_{13}=\frac{\tilde{n}_{1}-\tilde{n}_{3}}{\tilde{n}_{1}+\tilde{n}_{3}}$ are the Fresnel reflectance at the air/ $\mathrm{VO}_{2}$ and $\mathrm{VO}_{2} / \mathrm{Al}_{2} \mathrm{O}_{3}$ interfaces, $\tilde{n}_{2}=1$ is the air refractive index, $\tilde{n}_{3}=0.4+i 0.06$ is the refractive index of $\mathrm{Al}_{2} \mathrm{O}_{3}$, and $\tilde{n}_{1}=\sqrt{\tilde{\varepsilon}_{1}}$ is the refractive index of $\mathrm{VO}_{2}$. Using the literature reported permittivity $(5.4+i 0.3)$ of MIphase $\mathrm{VO}_{2}{ }^{41,42,44,196,204}$, the relative reflectance expected for a MM-phase permittivity of $\tilde{\varepsilon}_{1}=-21+i 76$ is $R_{M M} / R_{M I}=0.47$, whereas the corresponding value for $\tilde{\varepsilon}_{1}=-30+i 1.5$ is $R_{M M} / R_{M I}=1.5$. Apparently, the first solution of $\tilde{\varepsilon}_{1}=-21+i 76$ is the only one that is consistent with the experimental reflectance measurements. This permittivity value in the MIR is very close to literature values of the RM-phase as can be seen in Table 4.1. According to the optical reflectance measurements, the MM and RM-phases behave optically similar throughout the entire spectrum from visible to MIR (figure 4.3), and the RM-phase permittivities are well documented.
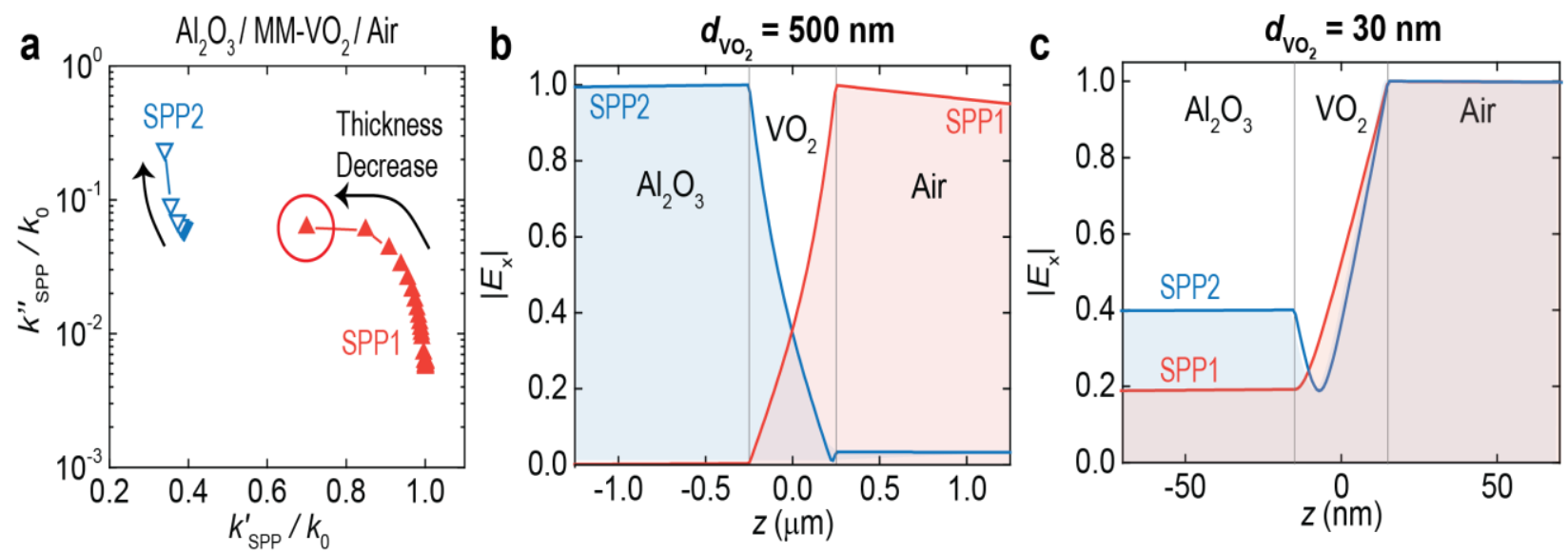

Figure 4.4 Film thickness dependence of SPP mode (a) The evolvements of the two thin-film SPP modes in $\mathrm{Al}_{2} \mathrm{O}_{3} / \mathrm{MM}-\mathrm{VO}_{2} /$ Air tri-layer structures as the $\mathrm{VO}_{2}$ film thickness decreases from $1 \mu \mathrm{m}$ to $30 \mathrm{~nm}$. The red circle marks the mode observed experimentally. $(\boldsymbol{b}, \boldsymbol{c})$ Field profiles of the two SPP modes along the out-of-plane direction in $500 \mathrm{~nm}$ films (b) where the mode coupling is weak, and in $30 \mathrm{~nm}$ films (c) where the coupling is strong. 
When the $\mathrm{VO}_{2}$ film is thin, the strong field overlapping between the $\mathrm{VO}_{2} /$ air and $\mathrm{VO}_{2} / \mathrm{Al}_{2} \mathrm{O}_{3}$ interfaces causes the two SPP modes to strongly hybridize and become sensitively dependent on the film thickness. Dispersion relations of the two modes were calculated using the extracted permittivity for the thickness range of $30 \mathrm{~nm}-1 \mu \mathrm{m}$ (figure 4.4a). Their out-of-plane field profiles at two representative thicknesses are also modeled and shown in figure 4.4b,c. The experimentally detected SPP mode (SPP1) forms from the antisymmetric coupling between the two interfaces. For small $\mathrm{VO}_{2}$ film thicknesses, the field of SPP1 significantly leaks into the substrate (red curve, figure 4.4c) and thus experiences a strong attenuation due to the phonon resonances of $\mathrm{Al}_{2} \mathrm{O}_{3}$ at MIR frequencies ${ }^{205}$. When the $\mathrm{VO}_{2}$ film becomes thicker, SPP1 will evolve into the regular bilayer ( $\mathrm{VO}_{2}$ /air) mode with a much longer propagation length $(\sim 140 \mu \mathrm{m})$. Other than SPP1, a second mode (SPP2) with $k=(0.34+i 0.24) k_{0}$ can also be supported by $30 \mathrm{~nm} \mathrm{VO}_{2}$ films which, however, is much more heavily attenuated.

\subsection{Demonstration of $\mathrm{VO}_{2}$ Nanophotonic Devices}

This SPP resonance of the MM-phase creates an opportunity for photonic or plasmonic device application. For the remainder of chapter 4, I will be exploring these opportunities with example devices. One option I explore is using the MM-phase in combination with other plasmonic materials to spatially modulate the plasmonic resonances in an on-demand manner. As shown in figure 4.5, arrays of gold gap-antennas were fabricated on the $\mathrm{VO}_{2}$ surface by electron beam lithography at Pittsburgh University. Each antenna is constructed from a pair of gold bars with a total end-to-end length of $8 \mu \mathrm{m}$ and a center gap that is $50 \mathrm{~nm}$ wide. Under the illumination of $\lambda=10.9 \mu \mathrm{m}$ MIR light, the plasmonic charge oscillations in the gold strips are expected to constructively couple together to produce a local near-field "hot spot" inside the gap.

Although the properties of the gold strips are not tunable, the plasmonic resonance inside the gap can be controlled by altering the surrounding environment ${ }^{206}$. According to finite-difference time-domain (FDTD) simulations (figure 4.5c), an intense in-gap "hot-spot" with more than 10-times field enhancement will be present when the $\mathrm{VO}_{2}$ film around the antenna remains in the dielectric MI-phase. In contrast, when the $\mathrm{VO}_{2}$ film nearby is converted to the metallic MM-phase, the field enhancement inside the gap will be suppressed. There is no longer a gap antenna, it becomes one continuous plasmonic bar. 


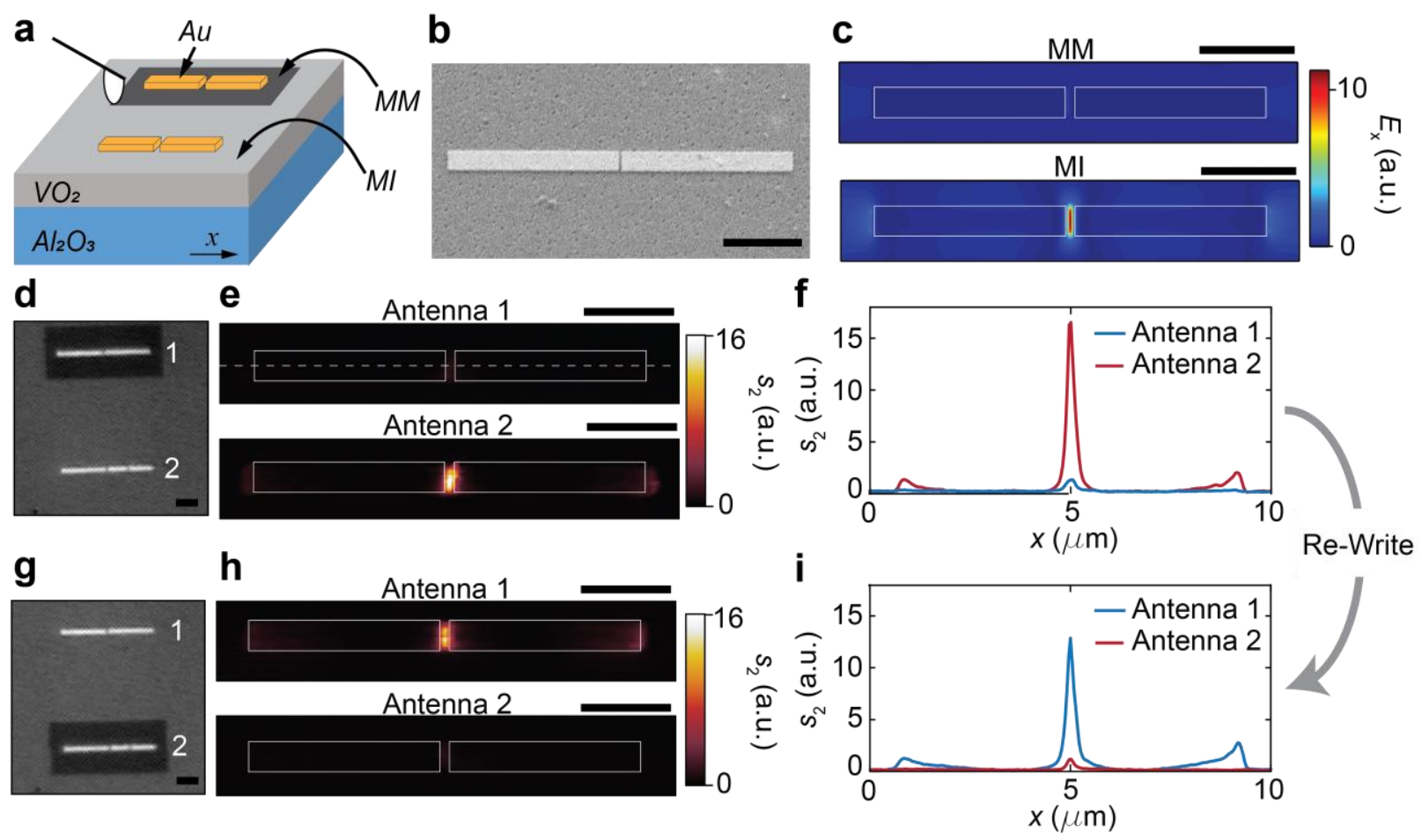

Figure 4.5 Spatially modulated plasmonic resonances (a) Illustration of a scanning probe locally inducing the MM phase around an Au gap-antenna. (b) Scanning electron microscope image of the gap antenna on $\mathrm{VO}_{2}$ film surface. (c) FDTD simulations of the in-plane field distributions at a detection plane $60 \mathrm{~nm}$ above the antennas surrounded by MM-phase (top) or MI-phase (bottom) $\mathrm{VO}_{2}$. (d) Optical images of two simultaneously fabricated antennas placed next to each other. Antenna-1 is surrounded by AFM-written MM-phase $\mathrm{VO}_{2}$, while the $\mathrm{VO}_{2}$ film near antenna-2 is left in as-grown MI phase. (e) SNOM images of the two antennas as shown in (d). Local "hot spot" is only detected in antenna 2. (f) SNOM line profiles measured along the two antennas. (g-i) Same as (d-f) but after an AFM-rewrite that erases the MM-phase rectangle surrounding antenna-1 and has another one patterned near antenna-2. Scale bars in this figure all represent $2 \mu \mathrm{m}$.

The idea is that when the $\mathrm{VO}_{2}$ material phase near each antenna can be individually controlled (figure 4.5a), spatial modulation of the field profile in the antenna array can be effectively achieved and reconfigured. Figures $4.5 \mathrm{~d}-\mathrm{i}$ demonstrate such modulations on a pair of gap antennas that are placed next to each other. During the first round of the experiment, a MM-phase rectangle is patterned around antenna- 1 as can be seen in figure $4.5 \mathrm{~d}$. Then the region near antenna- 2 is left in its as-grown state. SNOM images of the antenna pair were carried out after the AFM writing 
process. The SNOM image agrees almost perfectly with the FDTD model (figure 4.5e): a strong near-field hot-spot is clearly visible inside the gap of antenna-2 but absent from antenna-1. Comparing the SNOM line profiles (figure 4.5f), an in-gap near-field signal ratio of 13 is observed between the two antennas. I note that in order to faithfully image the in-plane field profile of the plasmonic resonance induced by the gold strips and minimize extrinsic perturbations caused by the scanning probe itself ${ }^{207,208}$, a bare silicone probe was used specifically for this experiment. For the other SNOM measurements discussed in this dissertation where the selection of particular field component is not critical, platinum coated probes were used to ensure optimized signal-to-noise ratio. Next the sample is heated to erase the MM-phase around antenna-1. Then the MM-phase is patterned around antenna-2 (figure $4.5 \mathrm{~g}$ ) and the same experiments are repeated. As expected, the near-field signals obtained from the two antennas simply trade places. This style of nanoplasmonic device has potential for on-chip logic. Individual circuits could spatially be modulated on and off. Also photonic encryption and communication could be patterned or encoded by the individual pixels ${ }^{209}$.

A second application option is to use the MM-phase as a monolithic platform for the fabrication of programmable on-chip planar optics ${ }^{58,64,210}$. Planar optics can refer to any ultra-thin optical element for manipulating far-field or near-field light. They can operate on various principles such as a refractive index contrast or gradient patterned into a film to accumulate a phase lag similar to common refractive optical elements ${ }^{211}$. Here I exploit the plasmonic absorption of the MM-phase to control the light. As an example, a reconfigurable wire-grid polarizer ${ }^{64,212,213}$ is demonstrated in figure 4.6. A one-dimensional array of MM-phase wires was created on the MI-phase background by the writing process (figure 4.6a). When the spacing between the neighboring wires is much less than the optical wavelength, the incident light sees the region as a homogenous but anisotropic media. The large wavelength light cannot distinguish the individual wires. When the incident light is polarized parallel to the MM-phase wires, mobile carrier oscillations along the wires are strongly coupled to the light field, producing an effective index similar to the value exhibited by solid metal films ${ }^{64,212,213}$. When the light polarization is perpendicular to the wires, the carrier oscillations across the insulating gaps is prohibited, a very different optical reflectance is produced. The reflectance behaves closer to the MI-phase. 


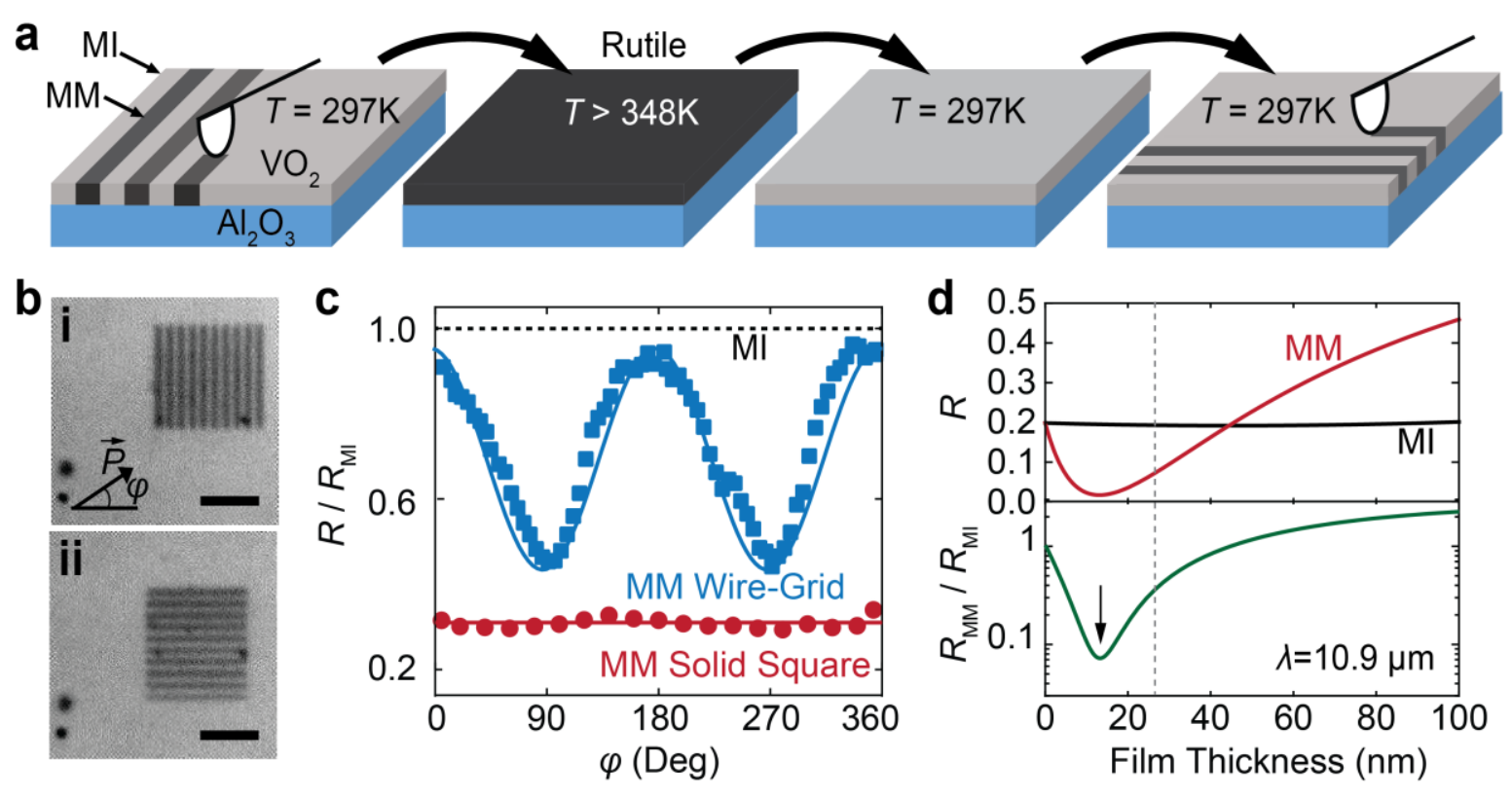

Figure 4.6 Reconfigurable wire-grid polarizer (a) Writing, erasing, and reconfiguration of MM-phase wire-grid polarizers. (b) Optical images of two wire-grid polarizers with same wire spacing $(900 \mathrm{~nm})$ but orthogonal optical axes that are created at the same location sequentially. Scale bars are $5 \mu \mathrm{m}$. (c) Polarization dependence of the wire-grid reflectance measured at $\lambda=10.9 \mu \mathrm{m}$ (solid blue squares), normalized by the MI-phase reflectance. The solid blue line represents the sinusoidal fitting. Reflectance values measured on a solid MM-phase square are also shown. (d) Thickness dependent optical reflectance calculated for $\mathrm{MM}-\mathrm{VO}_{2}$ and $\mathrm{MI}-\mathrm{VO}_{2}$ films according to equation 4.6. The reflectance contrast between these two phases maximizes $\left(R_{M I} /\right.$ $R_{M M}=14$ ) at a film thickness of $13 \mathrm{~nm}$ (marked by the black arrow). The vertical dashed line marks the film thickness used in the current experiment.

Figure 4.6b shows the optical images of two such $\mathrm{MM}-\mathrm{VO}_{2}$ wire-grids with orthogonal polarization axes that were patterned in the same location sequentially following the procedures illustrated in figure 4.6a. The same wire spacing of $900 \mathrm{~nm}$ was used in both structures to support MIR operations. The two structures exhibit similar polarizer performances but with an expected $\pi / 2$ phase shift. Data measured in the first wire-grid is shown in figure 4.6c. The reflectance maximizes close to the MI-phase value when the incidence polarization is perpendicular to the wires and minimizes near the MM-phase value when they are parallel, showing an extinction ratio of 2.3. I note that the reflectance contrast between the MI-phase and MM-phase is strongly affected 
by the interferences of the light internally reflected inside the $\mathrm{VO}_{2}$ film ${ }^{197,204,214}$, and is thus highly sensitive to the film thickness (figure 4.6d). It is my expectation that the extinction ratio of MMphase wire-gird polarizers can be substantially improved by reducing the film thickness to $\sim 13 \mathrm{~nm}$, where the reflectance of the MM-phase is more than 14 times weaker comparing to the MI-phase.
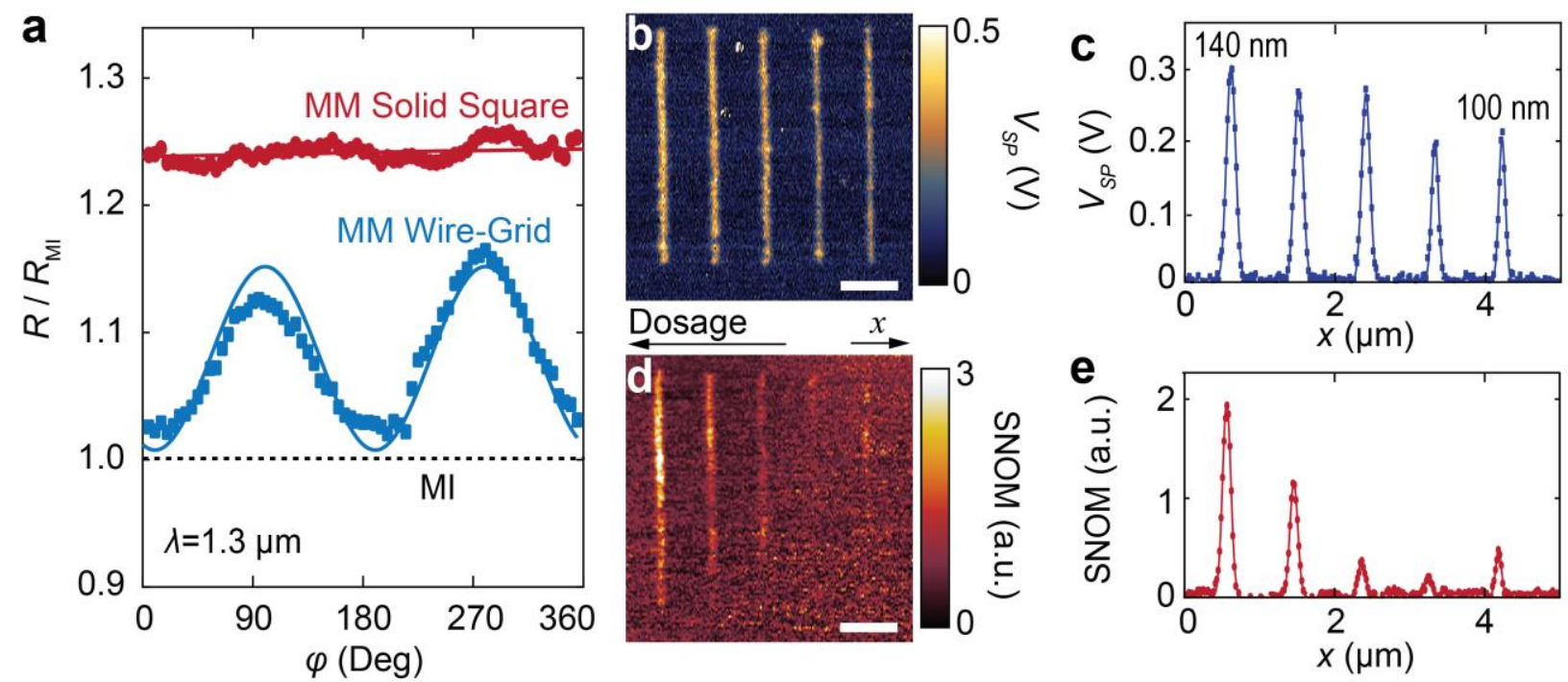

Figure 4.7 Size-dependent optical performance of $\mathrm{MM}-\mathrm{VO}_{2}$ structures (a) Polarization dependent telecom frequency reflectance measured on a wire-grid polarizer with a smaller wire spacing of $500 \mathrm{~nm}(\boldsymbol{b}, \boldsymbol{d}) \mathrm{KPFM}$, SNOM images simultaneously recorded for five parallel lines created with reducing AFM writing dosages. All the lines are written with the same tip bias of $10 \mathrm{~V}$. The tip scan speeds used for the five lines from left to right are $0.5 \mu \mathrm{m} / \mathrm{s}$ (double pass), $1 \mu \mathrm{m} / \mathrm{s}$ (double pass), $2 \mu \mathrm{m} / \mathrm{s}$ (double pass), $0.5 \mu \mathrm{m} / \mathrm{s}$ (single pass), $1 \mu \mathrm{m} / \mathrm{s}$ (single pass). Scale bars are 1 rm. (c, e) KPFM (c) and SNOM (e) line profiles across the five lines (solid dots) and the corresponding Gaussian fits (solid curve). The FWHMs of the lines range from $140 \mathrm{~nm}$ to $100 \mathrm{~nm}$.

It is possible to further shrink the characteristic dimensions of the $\mathrm{MM}-\mathrm{VO}_{2}$. Figure 4.7a demonstrates another wire-grid with a denser wire spacing $(500 \mathrm{~nm})$ that can function at the telecom frequency $(\lambda=1.3 \mu \mathrm{m})$. The RM-phase is actively plasmonic down to a wavelength of approximately $1 \mu \mathrm{m}$ making these plasmonic devices broadband ${ }^{41,42,44,196}$. To explore the smallest structure size achievable, a group of five lines were drawn with intentionally small AFM writing dosages. The written lines were then imaged by simultaneous SNOM and KPFM measurements that were discussed in section 2.5 (figure 4.7b-e). As shown in figure 4.7b, uniform work function 
profiles are found in all five lines. Their full widths at half maximum (FWHMs) range from 140 $\mathrm{nm}$ to $100 \mathrm{~nm}$, which reduce slightly to $137 \mathrm{~nm}$ and $95 \mathrm{~nm}$ after deconvolutions considering the probe radius of curvature $(\sim 30 \mathrm{~nm})$. While it is relatively easy to produce heavily doped structures with characteristic dimensions below $100 \mathrm{~nm}$, it is more challenging to maintain a reliable optical metallicity for such small scales. As shown in figure 4.7d, SNOM signal along the lines becomes highly non-uniform and falls quickly as the line width reduces below $140 \mathrm{~nm}$. Therefore, it is reasonable to set the size limit of $\mathrm{MM}$-phase $\mathrm{VO}_{2}$ optical structures at $150 \mathrm{~nm}$, which should adequately meet the requirements for most infrared plasmonic meta-devices and planar $\operatorname{optics}^{212,215}$. 


\section{Chapter 5}

\section{Oxygen Vacancy Dynamics in Monoclinic $\mathrm{VO}_{2}$}

At this point in this dissertation, all $\mathrm{VO}_{2}$ limitations have been addressed and solved except for the volatility or in other words stability of metallic phases. I have shown that the locally induced MM-phase has photonic and plasmonic application while having stable characteristics. However, stable characteristics is an unsatisfactory, vague statement. In this chapter, I will monitor and quantify the stability of the MM-phase. As discussed previously, the metallic domains are created by an injection of OVs. Thus the overall stability is based on the dynamics of the OVs from the initial injection site. Diffusion rates for various ionic species, including OVs, are documented for rutile $\mathrm{VO}_{2}$ but unexplored for the monoclinic lattice 104,216,217. Here I am exploring the OV dynamics in monoclinic $\mathrm{VO}_{2}$. I will identify $\mathrm{OV}$ depletion due to recombination with the atmosphere oxygen and quantify an in-plane diffusion constant for the OVs. Then I will model how these rates limit nanoscale device lifetimes.

The monoclinic a-axis (rutile c-axis) of the $30 \mathrm{~nm}$ films are oriented as the film out-of-plane. Field-effect OV injection is only successful along this axis due to the V-V chains and has been shown to have vacancy penetration depths greater than $0.5 \mu \mathrm{m}^{63,104}$. Thus the metallic domains induced here are assumed to be uniform throughout the entire $30 \mathrm{~nm}$ film thickness. The domain electrical and optical properties are monitored with respect to time to understand the OV dynamics. One propagation pathway is the OVs can diffuse out-of-plane along the monoclinic a-axis, back to the injection site at the film surface. Here the OVs can recombine with oxygen from the atmosphere to deplete the overall vacancy concentration as illustrated in Fig. $1 a^{218-220}$. Another option is for the OVs to diffuse in-plane. The gradient of vacancy concentration will redistribute the OVs throughout the entire MI film lattice.

Sequential KPFM scans can be used to monitor the $\mathrm{VO}_{2}$ surface potential over time to understand the OV dynamics. Doping semiconductors with donor ions such as OVs raises the Fermi level, tuning the surface potential and work function ${ }^{221,222}$. The change in surface potential due to OV doping is directly measured in the KPFM images. Then the relative carrier density of the MI and MM-phases can be extracted using equation $5.1^{216,222}$ : 
$n_{M M} / n_{M I} \approx \exp \left[e\left(V_{M M}-V_{M I}\right) / k_{B} T\right]$

where $n_{M M} / n_{M I}$ is the ratio of charge carrier density between the MM and MI-phases, $V_{M M}-V_{M I}$ the difference in surface potential, $T$ is temperature, $e$ is the charge of an electron, and $k_{B}$ is the Boltzmann constant.

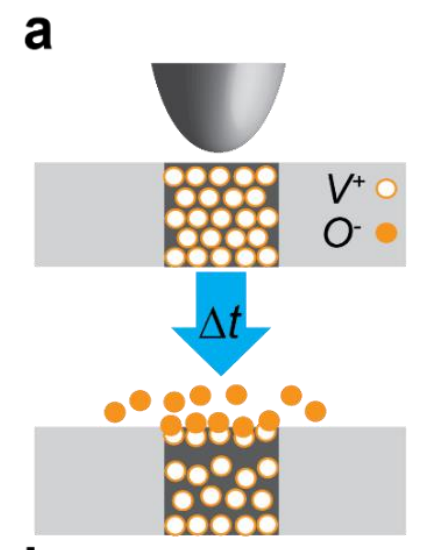

b
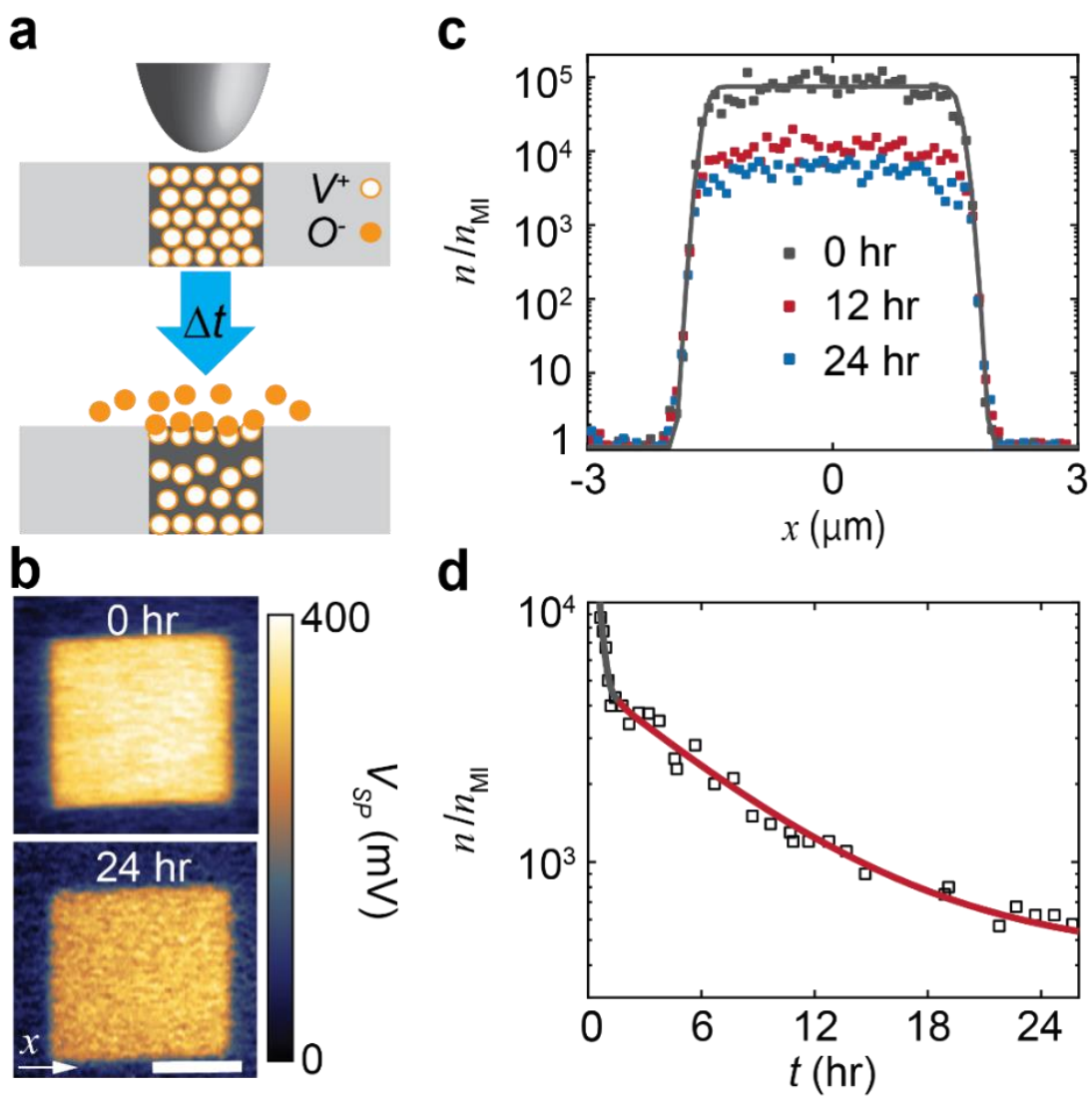

Figure 5.1 Oxygen vacancy depletion rates (a) Illustration of MM domain, via OV doping, written into the $\mathrm{MI} \mathrm{VO}_{2}$. Over time the OVs at the surface recombine with atmosphere oxygen while the remaining slowly diffuse towards the surface. (b) KPFM images of initial written MM domain and after 24 hrs. Scale bar represents $2 \mu \mathrm{m}$. (c) Spatial profile evolution of the relative carrier density over a 24 hour period. (d) Two-factor exponential decay fit for the depletion rate of the relative carrier density of the MM domain.

MM-phase domains were patterned in the film, and then the relative surface potentials of the MM and MI-phases were measured for over 24 hours (figure 5.1b). The writing process often causes degradation of the AFM cantilever due to the attraction of ionized particles ${ }^{223}$, therefore a 
new cantilever was implemented between the writing process and the KPFM measurements. The new cantilever along with identical measurement conditions were used throughout the entirety of the KPFM measurements for consistent results. The spatial profiles of the KPFM images at various times are converted into relative carrier density (Eq. 5.1) and plotted in figure 5.1c where "0 hr" represents the initially taken KPFM image. The obvious profile evolution that can be seen is the distinct depletion of the MM charge carrier density or OVs. Any in-plane spatial profile effects are negligible indicating a dominate out-of-plane depletion process. The depletion trend of the OVs with respect to time is then plotted in figure 5.1d. The depletion rate fits best to a two-factor exponential decay:

$n_{M M} / n_{M I} \sim \exp [-t / 0.3]+\exp [-t / 6.9]$

There is an initial fast process of carrier density decay; $\exp [-t / 0.3]$. This is attributed to the free carriers that were pulled to the film surface through field-effect during the writing process which quickly migrate back into the lattice, and also recombination with the atmosphere oxygen of the OVs localized at the film surface. This creates an OV density out-of-plane gradient due to the recombination which causes the remaining OVs to diffuse along the monoclinic a-axis towards the film surface. Upon reaching the film surface at a slower rate, $\exp [-t / 6.9]$, further OV recombination takes place ${ }^{218-220}$.

The optical property contrast between the MI and MM-phases in the visible regime allows for optical identification and monitoring of the as well ${ }^{42,87}$. To further verify that the OV depletion is due to the recombination of the OVs with oxygen from the atmosphere, an MM domain was written on "Day 0" and optically monitored under high vacuum conditions $\left(10^{-5} \mathrm{mbar}\right)$ in figure 5.2a. The $\mathrm{VO}_{2}$ sample was removed from the high vacuum chamber for about 20 minutes each day to obtain an optical image, and for 9 days there was no noticeable degradation to the MM domain. After the 9 days, the sample was removed from vacuum and continued to be optically monitored under ambient conditions (figure 5.2b). In this environment, noticeable changes to the optical contrast occur on a daily scale, and the lifetime of the metallic domain was approximately one week. This experiment provides evidence of the importance of the oxygen environment. The OV depletion rate can be nullified or at least greatly reduced if the sample surface can be isolated from oxygen molecules by vacuum or possibly sample capping. 


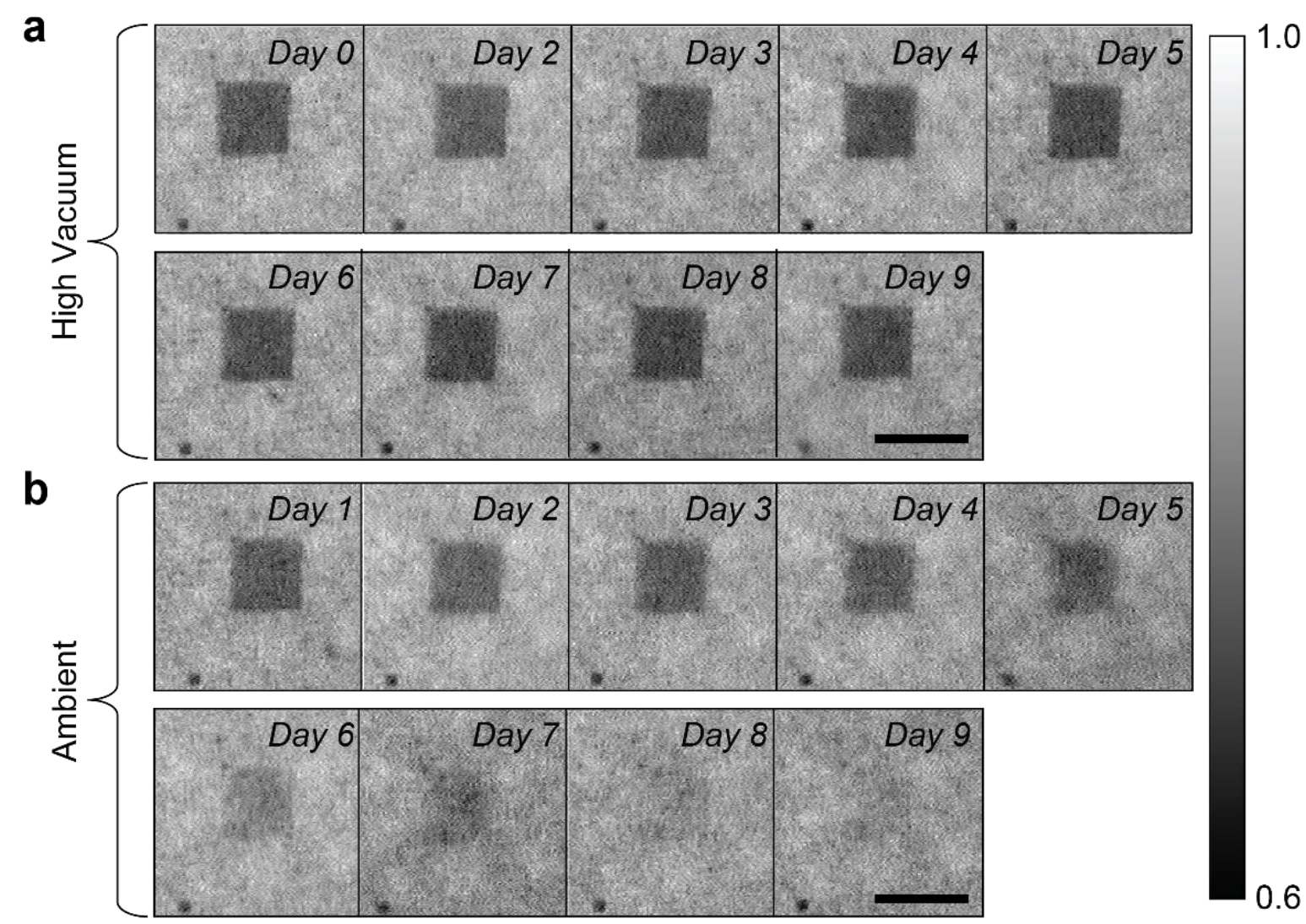

Figure 5.2 Environmental MM-phase stability (a) Optical images of metallic square written into the insulating $\mathrm{VO}_{2}$ sample and placed into a high vacuum chamber $\left(10^{-5} \mathrm{mbar}\right)$. Square monitored daily. (b) Sample was removed from vacuum and placed into ambient lab conditions (40\% RH, 20 $\left.{ }^{\circ} \mathrm{C}\right)$. The metallic domain lifetime was approximately 1 week. All scale bars represent $5 \mu \mathrm{m}$.

I now have an understanding of the dominate out-of-plane dynamics of the OVs, but a more detailed observation into in-plane diffusion still needs to be considered for vacuum and capped samples. In figure 5.3a, I illustrate the idea that the extreme gradient of defects can cause a radial in-plane diffusion. KPFM measurements were taken for over a $24 \mathrm{hr}$ period again, but this time on nano-scale MM wires (figure 5.3d). Using a wire makes the spatial width evolution a 1D problem that can be modeled by Fick's $2^{\text {nd }}$ law of diffusion 216224225 :

$\partial \mathrm{n}(\mathrm{x}, \mathrm{t}) / \partial \mathrm{t}=\mathrm{D} * \partial \mathrm{n}(\mathrm{x}, \mathrm{t}) / \partial \mathrm{x}$

$n(x, t)=\frac{A}{\sqrt{4 \pi D t}} \exp \left[-x^{2} /(4 D t)\right]$

$w=\sqrt{D t}$ 
where Eq. 5.3 is Fick's $2^{\text {nd }}$ law, Eq. 5.4 the solution of Fick's $2^{\text {nd }}$ law for a 1D Gaussian, and Eq. 5.5 the diffusion length of the carriers. $n$ represents the charge carrier density, $D$ the diffusion constant, and $w$ the full width at half maximum. Figure 5.3b plots the Gaussian fit across the width of the MM wire initially and after $24 \mathrm{hrs}$. The OV depletion occurs, but also an increase of the full width at half maximum, $w$. The evolution of $w$ with respect to time is plotted in figure $5.3 \mathrm{c}$ and fit using Eq. 5.5 to obtain $D=3.2 \times 10^{-16} \mathrm{~cm}^{2} / \mathrm{s}$. The KPFM images in figure $5.3 \mathrm{~d}$ shows that a perpendicular nano-wire was also monitored. The horizontal nano-wire fit to a diffusion constant $D=1.5 \times 10^{-16} \mathrm{~cm}^{2} / \mathrm{s}$ in figures $5.3 \mathrm{e}$,f. The diffusion constants are different by a factor of two but due to the resolution limitation of the measurement the diffusion is considered to be isotropic.

a
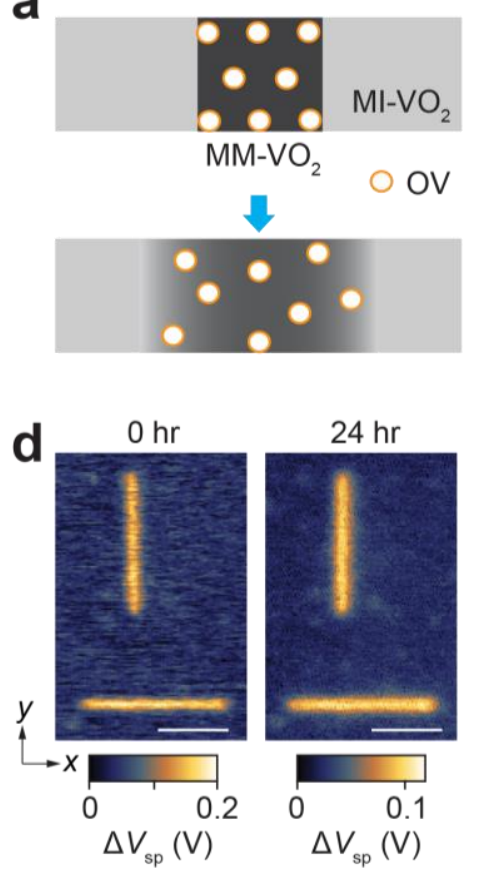

b
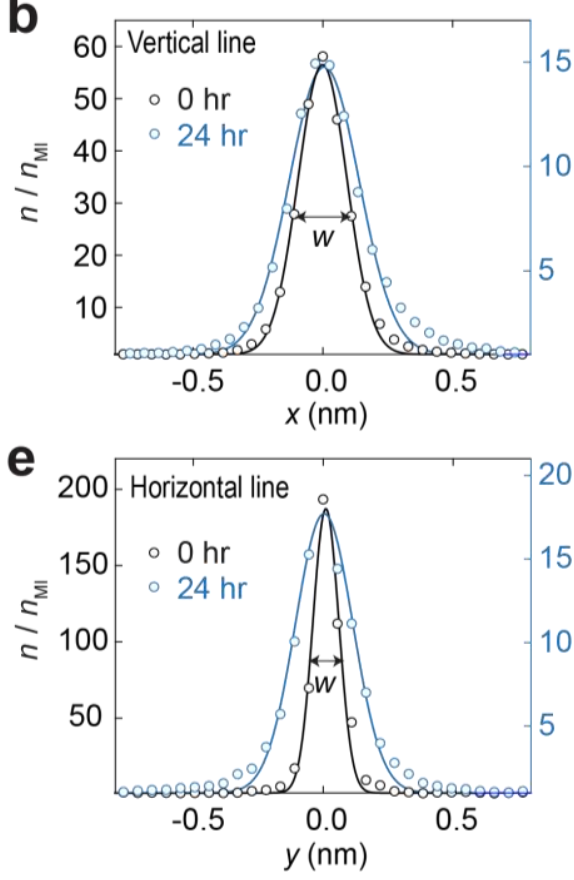
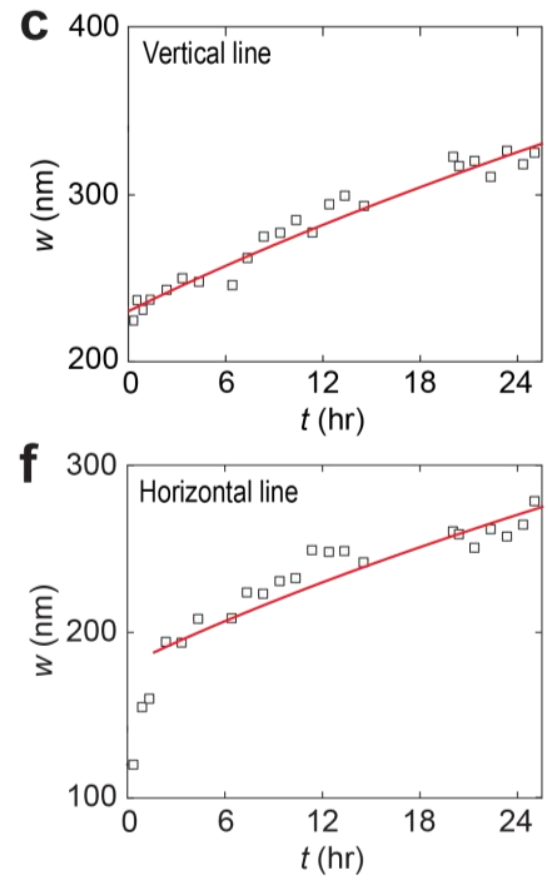

Figure 5.3 In-plane oxygen vacancy diffusion (a) Illustration of the OVs of a written MM-domain diffusing into the surrounding MI-film. (b) Gaussian fit of relative carrier density line cut across $200 \mathrm{~nm}$ nano-wire initially and after 24 hrs. (c) The full width at half maximum, w, of the MM nano-wire vs time. Fit to a square root dependence, $w=\sqrt{D t}$, to obtain the in-plane diffusion constant, $D=3.2 \times 10^{-16} \mathrm{~cm}^{2} / \mathrm{s}$. (d) KPFM images of initial MM nano-wire and after $24 \mathrm{hrs}$ along with another perpendicular nano-wire. Scale bars represent $2 \mu \mathrm{m}$. (e) Gaussian fit of relative carrier density line cut across perpendicular nano-wire initially and after $24 \mathrm{hrs}$. (f) The full width at half maximum, $w$, of the perpendicular nano-wire vs time; $D=1.5 \times 10^{-16} \mathrm{~cm}^{2} / \mathrm{s}$. 
A second geometry was made to confirm the measurements of depletion and $D$. An array of MM nano-wires were written to enhance the sensitivity of the measurements as can be seen in figure 5.4a. In this geometry depletion and diffusion are taking place, but the diffusion is causing the nano-wires to merge together. The valleys between the nano-wires are actually gaining OVs as can be seen in figure $5.4 \mathrm{~b}$. The line profile of the relative carrier density shows that the Gaussian peaks are decreasing, but the valleys are increasing in relative OVs with respect to time. Figure $5.4 \mathrm{c}$ plots the difference in the peak to valley relative carrier density with respect to time. This is then fit using the calculated diffusion constant, $D$, multiplied by the depletion rate:

$n(x, t)=\frac{A_{1}}{\sqrt{4 \pi D(t+\Delta t)}} \exp \left[-x^{2} /(4 D(t+\Delta t))\right]\left(A_{2} \exp [-t / 0.3]+A_{3} \exp [-t / 6.9]\right)$

where $A_{2}$ and $A_{3}$ are known from the depletion rate fitting, $A_{1}$ is used to fit the initial condition amplitude, and $\Delta \mathrm{t}$ is used to fit the initial condition width. The fitting agrees well with the experimental data further confirming the quantified depletion and diffusion rates.
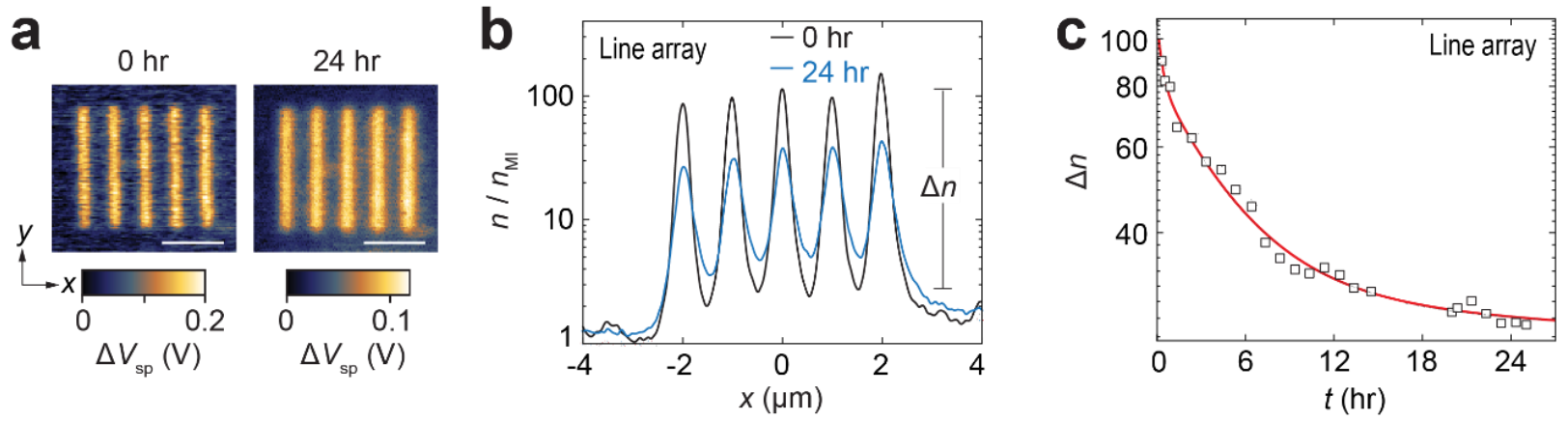

Figure 5.4 Nano-wire array depletion \& diffusion (a) KPFM images of initial MM nano-wire array and after $24 \mathrm{hrs}$. Scale bars represent $2 \mu \mathrm{m}$. (b) Line cut of relative carrier density across nano-wire array initially and after 24 hrs. (c) The difference of relative carrier density of array peaks and valleys with respect to time.

The value of $D$ also agrees with the minimal literature on monoclinic $\mathrm{VO}_{2}$ diffusion. It has been estimated that the hydrogen diffusion constant is approximately 3 orders of magnitude slower in monoclinic than rutile $\mathrm{VO}_{2}{ }^{217}$, and $D$ of OVs in rutile $\mathrm{VO}_{2}$ has been reported on the order of $10^{-13} \mathrm{~cm}^{2} / \mathrm{s}^{104} 216$. This drastic increase in $D$ is why annealing the sample erases the MM domains ${ }^{87}$. The OVs can rapidly escape the domain once the rutile phase is activated as shown in figure 5.5. Optical images were taken of a large $8 \mu \mathrm{m}$ MM square for increasing annealing 
temperatures. The sample was placed on a hotplate for 10 minute increments at the increasing temperature steps. Between increments the sample was removed from the heat for the optical image. No optical contrast effect is noticeable as long as the temperature driven IMT is not activated. Upon reaching the rutile phase transition, the MM domain rapidly diffuses away.

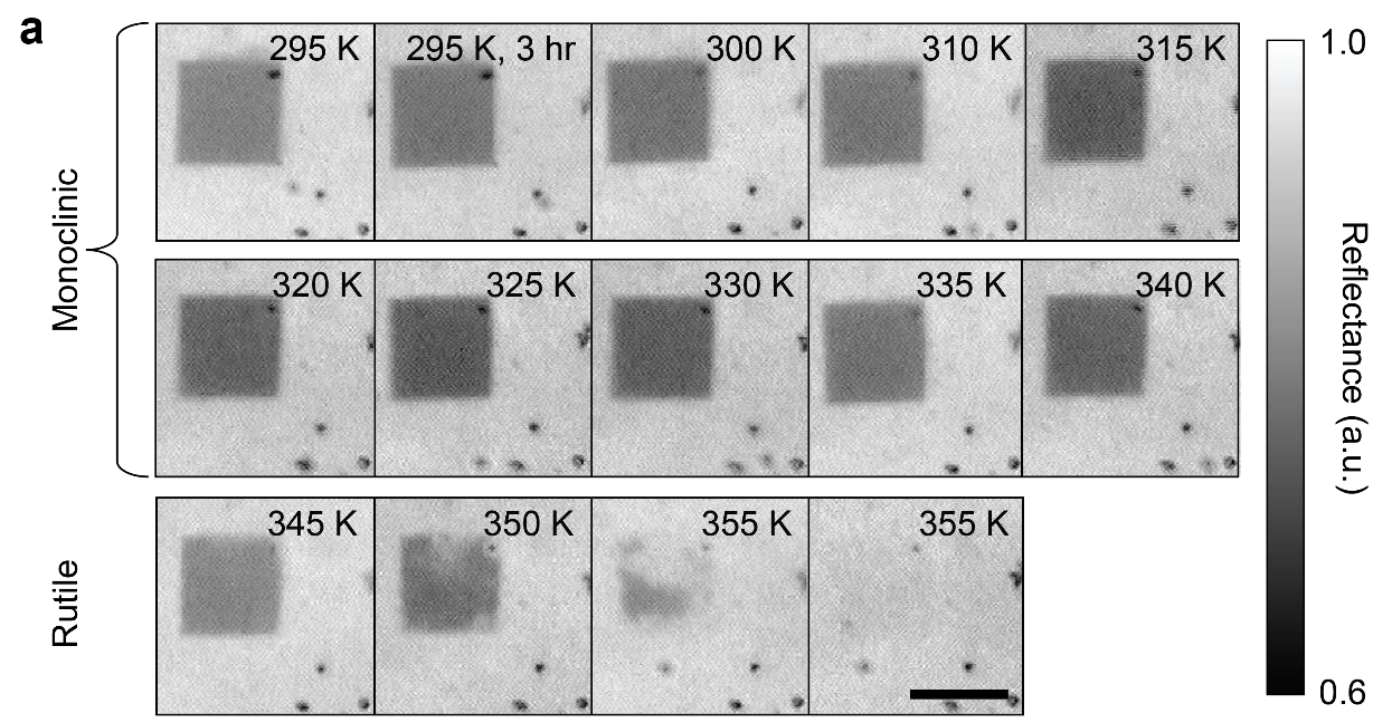

b

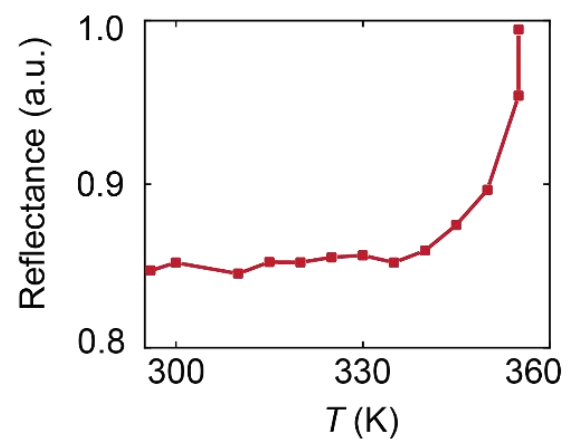

Figure 5.5 Monoclinic vs rutile metallic domain dynamics (a) A MM square monitored as a function of annealing temperature. Rutile phase activates the OV diffusion. Scale bar represents 8 $\mu m$. (b) The relative reflectance of $M M$ and $M I \mathrm{VO}_{2}$ for the optical images in (a).

Other shapes can also be used besides nano-wires. An array of nano-dots were induced using voltage pulses with various amplitudes and durations to understand writing dosages. The AFM tip was placed in contact with the sample, a voltage pulse was applied, and the cantilever was lifted and moved to the next nano-dot location. Immediately after, the AFM was switched into the in situ KPFM and SNOM imaging mode which acquired figures 5.6a,b simultaneously. The surface potential and metallicity of the nano-dots could then be correlated in figure 5.6c. At weaker writing 
dosages, no SNOM signal was detected indicating a lack of metallicity. With stronger writing dosages, there is a linear correlation trend with a threshold of a $190 \mathrm{mV}$ surface potential increase for the $\mathrm{VO}_{2}$ to become metallic.

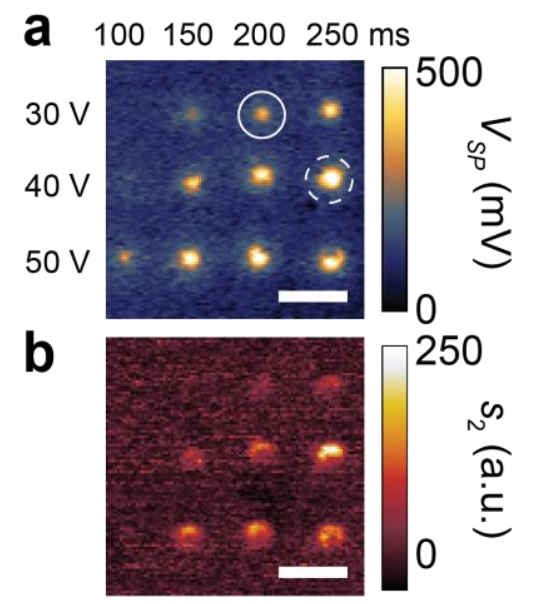

d

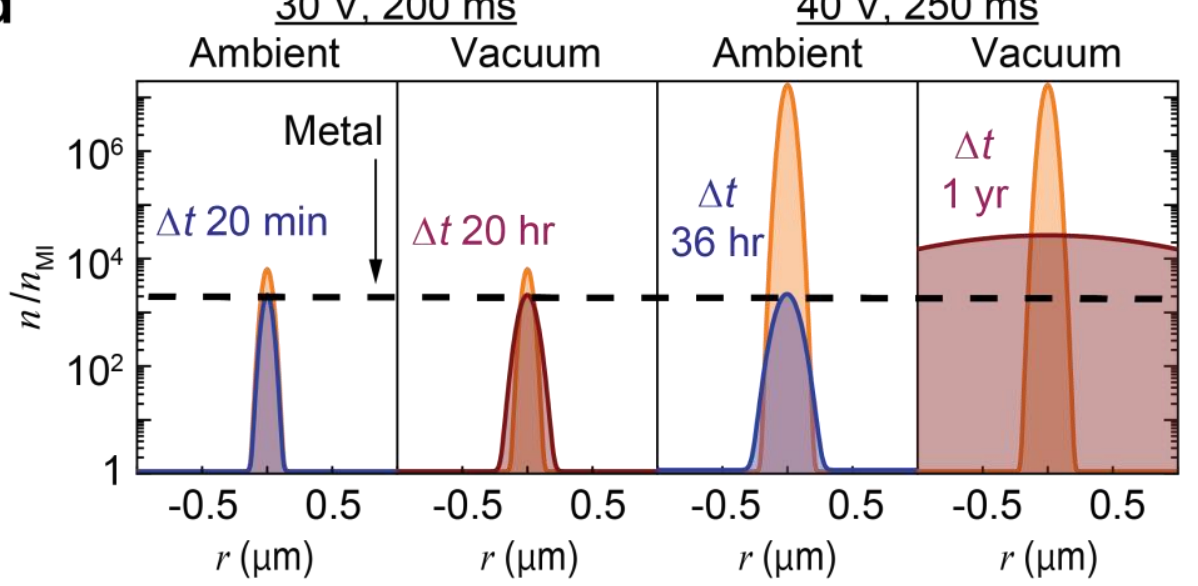

Figure 5.6 Metallicity correlation \& lifetime $(\boldsymbol{a}, \boldsymbol{b})$ In situ KPFM, SNOM images obtained of nanodots induced by an array of voltage pulses. Scale bars represent 1 Mm. (c) Correlation plot between $V_{s p}$ and $S_{2}$ in images $a, b$. An induced surface potential increase of $190 \mathrm{mV}$ is required to activate the MM phase (d) Dynamics model for the nano-dots circled in a,c. The quantified diffusion and depletion rates are used to predict nano-dot lifetimes in ambient conditions and vacuum. Dashed line represents the MM threshold in $c$.

Particular attention needs to be paid to the in-plane diffusion when using the MM-phase to create nano-devices. For example, metasurfaces are commonly comprised of nano arrays of metallic elements. The OV diffusion could begin to blend the elements together, eliminating the 
clear metal-dielectric contrast similar to the nano-wire array in figure 5.4. The quantified depletion and diffusion rates allow for the temporal and spatial evolution of MM domains to be modeled for future nano-devices in figure 5.6d. As an example, the exact width and amplitude of the circled nano-dots in figure 5.6a,c were used as initial conditions in the radial equivalent of the 1D diffusion model shown earlier (Eq. 5.6).

$n(r, t)=\frac{A_{1}}{4 \pi D(t+\Delta t)} \exp \left[-r^{2} /(4 D(t+\Delta t))\right]\left(A_{2} \exp [-t / 0.3]+A_{3} \exp [-t / 6.9]\right)$

Similar to the nano-wires in figure 5.4, the initial conditions were set using $\mathrm{A}_{1}$ and $\Delta \mathrm{t}$. The weaker nano-dot written with $30 \mathrm{~V}$ for $200 \mathrm{~ms}$ remains metallic for only 20 minutes. The stronger nano-dot written with $40 \mathrm{~V}$ for $250 \mathrm{~ms}$ has a lifetime of 36 hours. However, if the depletion process is absent because the sample is capped or in vacuum, these lifetimes improve to 20 hours and about 12-13 years. The diffusion process minimally effects the spatial profile in ambient conditions because the depletion process dominates. However in vacuum, future nano-devices will need to account for spatial spread. The nano-dot diffusion can clearly be seen in the right panel of figure $5.6 \mathrm{~d}$ representing the $40 \mathrm{~V}, 250 \mathrm{~ms}$ nano-dot in vacuum.

According to my model the key to long lifetime MM domains is a high relative OV density that penetrates deep into the $\mathrm{VO}_{2}$ film. Stronger writing dosages increase the relative $\mathrm{OV}$ density but also the size of the MM domains. To create smaller metallic domains with long life-times, the electric field divergence needs to be suppressed during the writing process. This would allow stronger writing dosages without doping larger areas. Shielded cantilevers ${ }^{226}$ and filament based phase change cantilevers ${ }^{227}$ have both been used to increase spatial resolution in scanning probe based electric field experiments.

I have investigated the mechanisms in which OV rich domains can dissipate in monoclinic $\mathrm{VO}_{2}$ to understand their stability. There is a depletion and in-plane diffusion of the OVs. The depletion can be minimized by isolating the sample surface from excess oxygen. With the obtained rates, I am able to model the life-time of MM domains. The lifetime has a large spread from minutes to years which if used correctly can be utilized in nanotechnology. 


\section{Chapter 6}

\section{Summary}

The unique properties of phase change materials such as Vanadium Oxides have been studied since the 1960's with great intrigue ${ }^{70,76,228-231}$. Now with the advancements in thin film fabrication processes $^{50-56}$, these phase change materials are being incorporated into complex heterostructures as active layers ${ }^{22,24}$. The active layers are used to tune or modulate existing photonic devices ${ }^{32,33}$. Specifically, GST phase transitions are utilized commercially for optical data retention ${ }^{38,59-61} \cdot \mathrm{VO}_{2}$ is regarded as the most studied phase change material, and has similarly drastic phase change properties to $\mathrm{GST}^{41-45}$. However as discussed previously, $\mathrm{VO}_{2}$ has three limitations for current technology implementation.

1. The $\mathrm{VO}_{2} \mathrm{IMT}$ is not controlled under ambient conditions in an on demand manner.

2. The $\mathrm{VO}_{2}$ IMT is a volatile phase transition which is not energy efficient over repeated cycles and temporal evolution.

3. $\mathrm{VO}_{2}$ phase transitions are activated on a macroscopic scale.

In recent years much work has been dedicated to manipulating $\mathrm{VO}_{2}$ using a variety of stimuli besides thermal activation. Most notably doping ${ }^{88-90,93}$ and $\operatorname{strain}^{81,86,87}$ have been used to modify the IMT $\mathrm{T}_{\mathrm{c}}$ on a macroscopic scale. Even defect engineering has been utilized to adjust $\mathrm{T}_{\mathrm{c}}$ locally on the microscale, although a volatile temperature driven process is still required overall ${ }^{64}$. Electrolyte gating has drawn the most attention, demonstrating macroscopic, reversible, stable phase transitions under ambient conditions excluding the electrolyte gel ${ }^{62,63,82,87,98,99}$. To this date, all phase transition efforts have addressed $\mathrm{VO}_{2}$ limitations individually. The work done in this dissertation addressed all three bullet points and solved at least 2 out of the 3 limitations, arguably all 3 depending on the criteria for non-volatile vs volatile phase transitions.

First a home-built SNOM system was engineered to address the $\mathrm{VO}_{2}$ material system. A resistive heating element attached to the base of the sample holder allows the thermal IMT to be activated throughout measurements making this SNOM system uniquely equipped for studying $\mathrm{VO}_{2}$. The SNOM imaging mode shows optical contrast between insulating and metallic domains. Also SNOM famously allows for wavelength independent spatial resolution determined by the 
size of the AFM tip apex which is $30 \mathrm{~nm}$ in the current work ${ }^{146}$. This SNOM system has the full capacities of a commercial AFM allowing electrical measurements such as KPFM to diagnose surface potential and work function. KPFM was even programmed for in situ measurement with SNOM for correlating the dielectric properties, work function, and topography of the $\mathrm{VO}_{2}$ phase transitions.

In this work, the AFM tip was used to apply various forms of scanning probe lithography to $\mathrm{VO}_{2}$ thin films grown on $\mathrm{Al}_{2} \mathrm{O}_{3}$. In one experiment strain was locally applied to the film by the scanning probe, decreasing $\mathrm{T}_{\mathrm{c}}$ in the selected region ${ }^{87}$. By decreasing $\mathrm{T}_{\mathrm{c}}$ lithographically, the selected region transitions into the $\mathrm{RM}$ phase while the rest of the as grown $\mathrm{VO}_{2}$ remains in the MI phase at the proper temperature. This experiment only successfully addressed bullet point 3 of $\mathrm{VO}_{2}$ limitations, localized phase manipulation.

Another experiment performed reduction scanning probe lithography. This idea stemmed from the success of the electrolyte gating of $\mathrm{VO}_{2}$ thin films. In electrolyte gating the large electric field generated by the electrolyte gel led to OV doping of the film which induced the $\mathrm{IMT}^{62,63,82,87,98,99}$. The Oxygen atoms of the film are able to combine with free electrolytes from the gel at the film surface under the large applied field. The reduction scanning probe lithography process is similar but locally applied. The nanoscale tip-sample junction was used to generate electric fields larger than $10^{9} \mathrm{~V} / \mathrm{m}$ only beneath the scanning probe. In ambient conditions $\mathrm{VO}_{2}$ is hydrophilic, and the water layer on the surface is ionized by the large field allowing it to act as the electrolyte gel. The field drives the migration of $\mathrm{OVs}$ into the $\mathrm{VO}_{2}$ activating an IMT while the $\mathrm{VO}_{2}$ remains in its monoclinic structure. This phase is known as the MM phase and is completely erased by annealing the sample above $\mathrm{T}_{\mathrm{c}}$. By reversing the applied bias, an insulating Van der Waals phase was induced. It was hypothesized that Hydroxyls may occupy the OV sites, but the insulating, amorphous phase has little applicable use and was not studied further.

The MM phase induced by reduction scanning probe lithography clearly solves bullet points 1 and 3 of $\mathrm{VO}_{2}$ limitations, on demand phase transitions in ambient conditions and $100 \mathrm{~nm}$ domain spatial resolution was achieved. The MM phase also showcased plasmonic optical behavior in the infrared. Plasmonic waveguides were used to diagnose the dielectric function at $10.9 \mu \mathrm{m}$ of the MM phase as $\varepsilon=-21+i 76$. The optical properties of the MM-phase are very similar to the RMphase. This plasmonic behavior allowed for the demonstration of $\mathrm{VO}_{2}$ spatial manipulations of 
resonant nanoplasmonic antennas for binary data retention. Specific antennas or pixels were modulated on and off. $\mathrm{VO}_{2}$ was also demonstrated as a wire-grid polarizer to propose the possibility of a monolithic platform for plasmonics and planar optics. The localized, on demand plasmonic phase in theory means that subwavelength, multifunctional metadevices could be patterned without the need for complex heterostructures. However, the MM phase propagation length in my experiments is only $14 \mu \mathrm{m}$ where as currently utilized metals such as Ag and Au have millimeter scale propagation lengths ${ }^{232}$. Although current analytical models do predict that a thicker $\mathrm{VO}_{2}$ film will increase the propagation length up to approximately $150 \mu \mathrm{m}$.

Despite the exciting behavior of the MM phase, it is not applicable if bullet point 2 of $\mathrm{VO}_{2}$ limitations is not addressed. The MM phase stability is based on the out of plane depletion through oxygen recombination at the sample surface and in plane diffusion of the OV's injected into the film. Relative OV density was monitored using KPFM measurements over 24 hour time periods. It was found that the depletion process dominates the lifetime of the MM domains but could be nullified with a vacuum or possibly sample capping. Depletion and diffusion rates were able to be quantified using the KPFM images. I was then able to model spatial and temporal evolution of nanostructures. This model will allow for the development of $\mathrm{VO}_{2}$ as monolithic platform for testing nanotechnology.

The work performed in this dissertation clearly solves the current hurdles in future $\mathrm{VO}_{2}$ technology through "On Demand Nanoscale Phase Manipulation of Vanadium Dioxide by Scanning Probe Lithography”. I demonstrated stable, nanoscale, metallic domains in ambient conditions. This work expands the capabilities of $\mathrm{VO}_{2}$ as an active layer in multifunctional device design, and provides the framework for a monolithic plasmonic platform.

\subsection{Outlook}

Current technology limitations often stem from the speed of data processing and transfer ${ }^{57,233}$. One solution being explored is further switching technology towards photonic integrated circuits $^{234}$. Photons in an optical medium are much faster than the drift velocity of electrons in today's semiconductor platforms ${ }^{235}$. Photons also offer information transfer with higher bandwidth and multiplexing capabilities ${ }^{57,236}$. In principle, photonic circuitry is faster and consumes less power than electronics. However, not all electronic building blocks have been achieved optically 
with high quality such as transistors and memory elements ${ }^{234}$. The ultrafast, drastic change in optical properties of phase change materials are now being used to demonstrate on-chip memory elements ${ }^{237,238}$. The change in transmission provides an optical read-out of the state similar to the logic of electronic memory elements. Also on demand, reconfigurable functionality provides reprogrammable logic ${ }^{239,240}$. The work in this dissertation makes $\mathrm{VO}_{2}$ a viable candidate for this logic operation. One experimental issue that usually arises is that most phase change materials are very lossy so the active layer is usually evanescently coupled to separate optical waveguides allowing transmission modulation without the attenuation. The tunability shown in this work for $\mathrm{VO}_{2}$ reflectance and plasmonic propagation length could circumvent this issue. For $\mathrm{VO}_{2}$ to be utilized as a memory element for photonic computing, continued research efforts will need to focus on producing a truly non-volatile phase transition for data retention.

Phase change materials are also coupled with plasmonic devices and metamaterials. Plasmonic materials are used to enhance electric fields at a metal-dielectric interface, and the functionality can be modulated similar to the Au nano-gap antennas demonstrated in chapter 4 of this work 241,242. As previously introduced for metamaterials, subwavelength arrays are used to create artificial optical phenomenon, but the function can be tuned by changing the background environment of the arrays ${ }^{24,25}$. Phenomenon such as negative refraction ${ }^{243}$ and perfect absorption 197,244 can be modulated on and off by using the phase transition. Planar lenses have their focal lengths tuned ${ }^{245,246}$, and absorption bands of sensors are shifted ${ }^{33,35}$. Metamaterial engineering is a fast growing topic with new devices being theorized every year. However, fabricating nanoscale arrays is time consuming, and the final product only serves a single function unless coupled to an active layer. A monolithic, plasmonic platform like $\mathrm{VO}_{2}$ adds a strategic advantage. The reconfigurable nature of the plasmonic entity creates a new metamaterial testing platform. New designs of plasmonic devices or metamaterials may be created on demand and reconfigured upon new product designs improving the pace of optical engineering research going forward. 


\section{Appendix A}

\section{Ferroelectric Domain Orientation in Multiferroic Thin Film BaCoF 4}

Scanning probe based technology has a wide range of uses. Here my goal is to demonstrate a different example of a unique measurement and manipulation using the scanning probe on multiferroic $\mathrm{BaCoF}_{4}$. In this section, I diagnose the orientation of in plane, nano-scale ferroelectric domains. Then I can pole the domains using an applied field.

Multiferroic materials exhibit exciting physical phenomena related to the simultaneous presence of multiple long-range orders, in many cases consisting of antiferromagnetism and ferroelectricity. To date, the majority of studies on multiferroic materials have been devoted to oxide perovskites in the form $\mathrm{ABO}_{3}$. The conventional ion transfer ferroelectricity mechanism in oxide perovskites is known to rely on the covalent bonds between oxygen and B-site cations with closed-shell, nonmagnetic electronic configurations ${ }^{247,248}$. Therefore, the search for magnetic ferroelectrics should be directed toward alternative ferroelectricity mechanisms. Alternatives can be found in the fluoride family which contains many examples of magnetic ferroelectrics ${ }^{249}$. The best known example is the $\mathrm{BaMF}_{4}$ compound, where $\mathrm{M}=\mathrm{Mn}, \mathrm{Fe}, \mathrm{Co}$, or Ni. Recent theoretical studies $^{250,251}$ focused on their magnetoelectric and multiferroic properties. To the best of my collaborators and my own knowledge, however, only studies of bulk form $\mathrm{BaMF}_{4}$ have been reported in the scientific literature ${ }^{249,252-262}$. Because thin films are essential for potential electronic device implementations, including heterostructure integration with existing architectures, it is important to determine whether these materials can be synthesized in thin film form, and whether the multiferroic behavior can be retained if growth strain is applied.

$\mathrm{BaCoF}_{4}$ thin films were grown for the first time under ultrahigh vacuum conditions using MBE by my collaborators at West Virginia University. Two constituents, $\mathrm{BaF}_{2}$ and $\mathrm{CoF}_{2}$, were coevaporated in 1:1 molar rates ${ }^{263}$. The choice of substrates suitable on which to grow $\mathrm{BaMF}_{4}$ films was nontrivial because the orthorhombic unit cell made it difficult to find a substrate with a good in-plane lattice match. For reference, the lattice parameters for $\mathrm{M}=\mathrm{Co}$ are $\mathrm{a}=4.21 \AA, \mathrm{b}=$ $14.63 \AA$, and $\mathrm{c}=5.85 \AA^{253}$. In these experiments, $\mathrm{Al}_{2} \mathrm{O}_{3}$ (0001) single crystalline substrates (hexagonal unit cell $\mathrm{a}=4.763 \AA$; $c=12.991 \AA$ ) were used ${ }^{263}$. 
These thin films underwent a thorough investigation of material properties to fully establish multiferroic behavior ${ }^{263}$. A brief overview is that structural characterization was performed by my collaborators using XRD to understand how strain due to the substrate effected lattice parameters. Due to the lattice mismatch with the (0001) $\mathrm{Al}_{2} \mathrm{O}_{3}$ substrates, the corresponding outof-plane b-axis and in-plane a-axis lattice parameters were smaller than in the bulk, while the inplane c-lattice parameter, representing the polar axis, was larger than in the bulk ${ }^{263}$. Next the magnetic nature of the film was established by my collaborators using a magnetic properties measurement system and a superconducting quantum interference device. The strained thin film maintained its antiferromagnetic structure and also showed new weak ferromagnetic transitions at low temperature ${ }^{263}$.
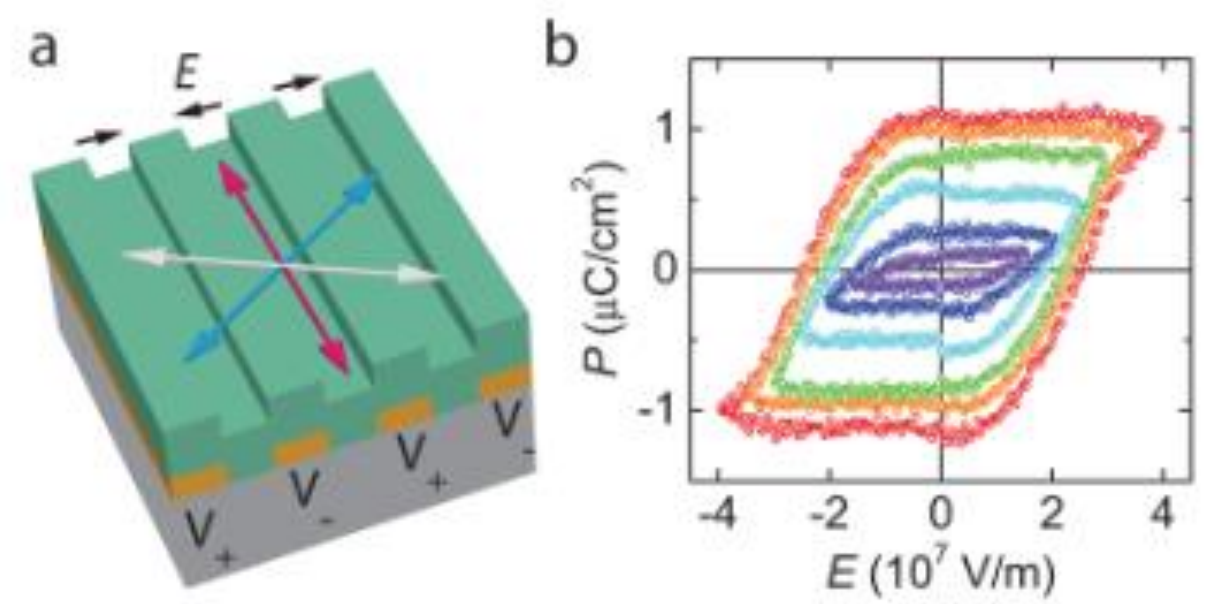

Figure A.1 Ferroelectric hysteresis measurements of $\mathrm{BaCoF}_{4}$ thin film (a) Interdigitated electrodes were deposited on the $\mathrm{Al}_{2} \mathrm{O}_{3}$ substrate and covered with the film. The E field direction was aligned perpendicular to one of the structural twin domains (polar c-axis noted as red arrow) and at $30^{\circ}$ with respect to the other two (blue and gray). Electrodes are separated by $2.5 \mu m$. (b) Remnant ferroelectric polarization vs applied electric in-plane field measured on a BaCoF $\mathrm{B}_{4}$ in film at $T=14 \pm 2 \mathrm{~K}$.

The focus of this section is to establish the ferroelectric behavior of $\mathrm{BaCoF}_{4}$ thin films and characterize the nano-scale ferroelectric domains using a scanning probe. $\mathrm{BaMF}_{4}$ compounds are expected to have ferroelectric polarizations along the crystallographic c-axis. In this case, that means the electric field should be applied in the film plane. This was achieved by depositing 
interdigitated electrodes directly on the substrate surface prior to the film growth (see figure A.1a). A separate sample without the electrodes was grown simultaneously in order to verify the film crystal quality from the XRD.

The electrodes were aligned such that the electric field direction applied during the measurements was perpendicular to the polar c-axis of one twin domain and at $30^{\circ}$ with respect to the other two domains (figure A.1a). Therefore, assuming the three in-plane crystal orientations are equally weighted, the measured response should be $(1 / 3)^{0.5}$, or approximately $58 \%$ of the single twin domain polarization. The leakage of the samples at room temperature was relatively high, and thus reliable ferroelectric hysteresis loops could not be measured. To minimize the leakage, ferroelectric measurements were performed at a temperature $\mathrm{T} \approx 14 \mathrm{~K}$.

Remnant hysteresis loops were measured up to a maximum electric field of $3.95 \times 10^{7} \mathrm{~V} / \mathrm{m}$, as shown in Figure A.1b. The remnant polarization of the largest loop was $\approx 1.1 \mu \mathrm{C} / \mathrm{cm}^{2}$, which was of the same order of magnitude as the $8.0 \mu \mathrm{C} / \mathrm{cm}^{2}$ measured in the bulk $\mathrm{k}^{256}$. Actual hysteresis loops have never been published for bulk $\mathrm{BaCoF}_{4}$, to the best of my knowledge. I therefore tentatively compare these loops with data reported for $\mathrm{BaZnF}_{4}{ }^{264}$. Typical coercive fields for $\mathrm{BaZnF}_{4}$ were reported to depend strongly on the ac field frequency, ranging from $1.0 \times 10^{6}$ to $6.0 \times 10^{6} \mathrm{~V} / \mathrm{m}$ for ac field frequencies between 0.01 and $100 \mathrm{~Hz}$, respectively. Since these measurements were performed with $100 \mathrm{~ms}$ electric field pulse duration, corresponding to a $10 \mathrm{~Hz}$ field frequency, a $3.35 \times 10^{6} \mathrm{~V} / \mathrm{m}$ coercive field would be expected if the compound were $\mathrm{BaZnF}_{4}$. In this case, the measured coercive fields were $\approx 2.5 \times 10^{7} \mathrm{~V} / \mathrm{m}$. In order to compare the two results, the semi empirical scaling law for the coercive field $E_{C}$ vs distance between contacts $d, E_{C}(d) \sim d^{-2 / 3}$, can be used $^{265}$. Using the separation between contacts of $2.5 \mu \mathrm{m}$, a calculation reveals that $\mathrm{BaZnF}_{4}$ should have $\mathrm{E}_{\mathrm{C}}=1.41 \times 10^{8} \mathrm{~V} / \mathrm{m}$ or approximately six times larger than the value measured in this $\mathrm{BaCoF}_{4}$ film. It is unclear whether this difference is due to an intrinsic property of the Co compound or if $\mathrm{E}_{\mathrm{C}}$ was reduced by the presence of defects in the film.

Regarding the maximum remnant polarization measured in $\mathrm{BaCoF}_{4}$, taking into account the presence of the three in-plane domains, the actual polarization should be 3 times the measured polarization or $\approx 2 \mu \mathrm{C} / \mathrm{cm}^{2}$. This is still lower than the value of $8.1 \mu \mathrm{C} / \mathrm{cm}^{2}$ measured in the bulk, 
but there are other factors that may have led to an underestimate; for example, there could be regions in the film that had larger switching fields than were able to be applied with this technique.
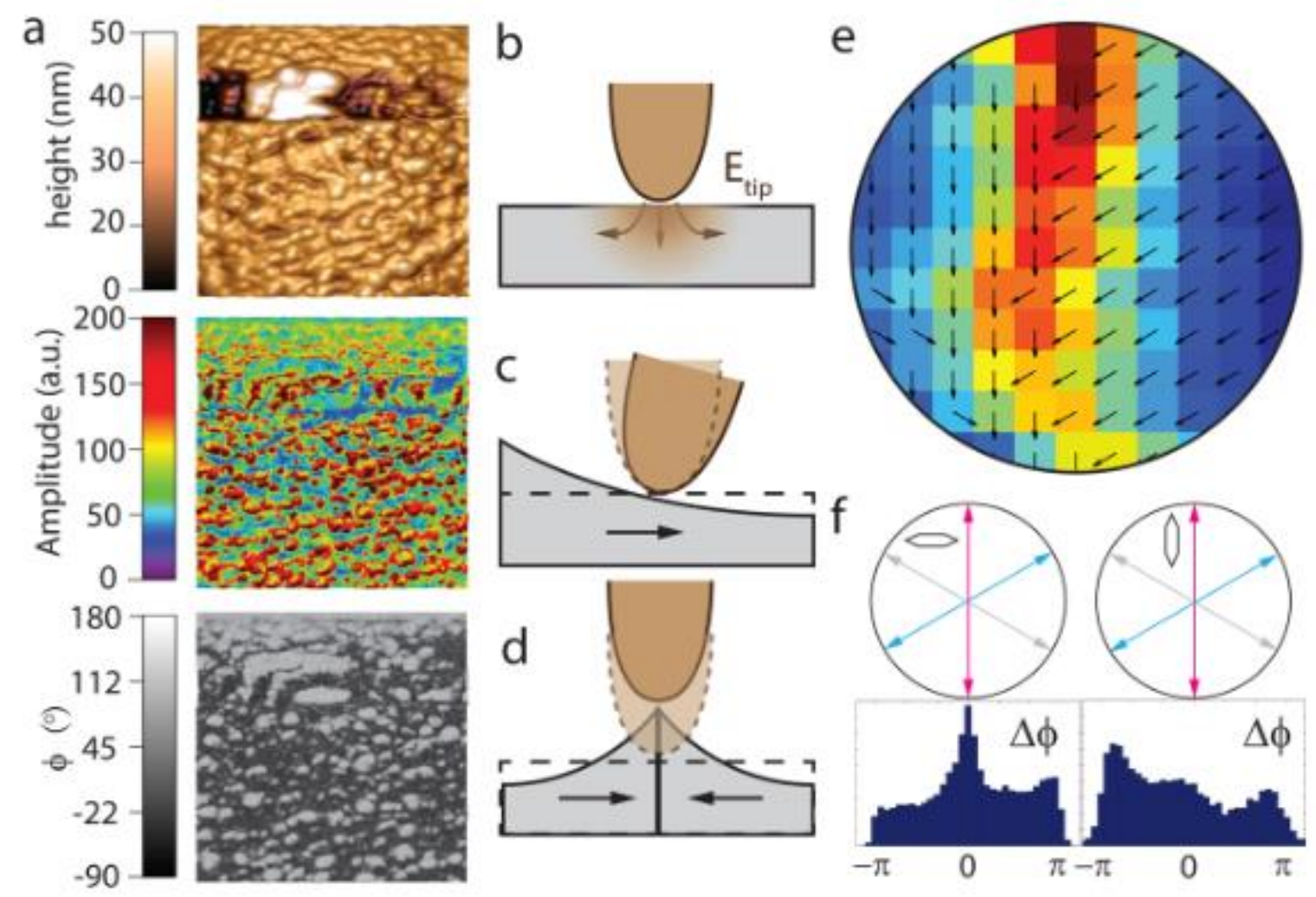

Figure A.2 PFM results for domain orientations of $\mathrm{BaCoF}_{4}$ thin film (a) Typical images of topography (top), PFM amplitude (middle), and PFM phase (bottom) in a $1.5 \mu \mathrm{m} \times 1.5 \mu \mathrm{m}$ area. (b) Schematic of the tip E field during PFM measurements. (c) Surface deformation when the tip scans the middle of an in-plane polarized domain. (d) Surface deformation when the tip scans over the boundary region between two domains with head-to-head polarizations. (e) Vector plot of local domain polarizations overlaid on top of the PFM amplitude intensity image. This information is extracted from a collection of four PFM measurements with different cantilever orientations. (f) Histograms of PFM phase change $\triangle \varphi$ after $180^{\circ}$ sample rotation for two orthogonal cantilever orientations noted by a small hexagon. When the cantilever is perpendicular to the polar c-axis in one of the structural twin domains (red), a large distribution at $\Delta \varphi=0$ is observed.

Pieoresponse Force Microscopy (PFM) ${ }^{266-270}$ was performed at room temperature to characterize the microscopic polar domains and ferroelectric properties of the $\mathrm{BaCoF}_{4}$ films. PFM supplies an AC bias to the probe which deforms a ferroelectric sample through the piezoelectric 
effect. A typical topography image and PFM responses are shown in figure A.2a. A root-meansquared surface roughness of $3.0 \mathrm{~nm}$ was observed across the sample, while the PFM responses revealed a granular sub $100 \mathrm{~nm}$ domain pattern. PFM data was acquired using the out-of-plane flexural vibrational mode of the cantilever. The in-plane torsional mode was also monitored but did not yield a significant signal, possibly due to the cantilever's high torsional rigidity. During the experiments, a small ac drive voltage of $1 \mathrm{~V}$ at the cantilever contact resonance frequency was applied to the buried electrode array, while the scanning probe was kept at the ground potential. Underneath the probe tip, a localized electric field $\left(E_{t i p}\right)$ was generated with both an out-of-plane field component and opposite in-plane components at opposing sides of the tip as shown in figure A.2b. The surface deformation of the film with in-plane polar c-axes in response to $E_{\text {tip }}$ is illustrated in figures A.2c and figure A.2d. In the middle of a domain, the out-of-plane deformation was small and mainly coupled to the cantilever's flexural mode through a weak shearing force (figure A.2c). However, at the boundary between two domains with head-to-head polarizations, the in-plane gradient of $E_{\text {tip }}$ induced a large out-of-plane deformation which strongly coupled to the cantilever's flexural vibration mode (figure A.2d). In the special case when the c-axis was perpendicular to the cantilever, the torsional shearing response was not detectable by flexural PFM.

The phase of the PFM signal induced by shearing force depended on the relative orientation between the local c-axis and the probe cantilever. In contrast, the PFM signal induced by out-ofplane deformations at domain boundaries was independent of such orientation changes. Therefore, information on the local c-axis orientations could be extracted by comparing the PFM phases after sample rotations (figure A.2e). Note that as expected, the overlaid PFM amplitude image in figure A.2e showed the strongest signals at domain boundaries. PFM results also supported the presence of three different c-axis orientations which was also deduced by my collaborators previously from XRD measurements. Figure A.2f shows the histograms of the phase differences $\Delta \varphi$ measured after rotating the sample by $180^{\circ}$ in two orthogonal configurations. The values of $\Delta \varphi$ were concentrated mostly around 0 and $\pm \pi$. Values around $\Delta \varphi=0$ corresponded to domain boundaries with no shear force and domains with polar c-axes perpendicular to the cantilever. The values around $\Delta \varphi= \pm \pi$ corresponded to domains with nonzero polarization components along the cantilever orientations. As expected, in the configurations when the cantilever was perpendicular to one of the c-axis orientations (colored red in Figure A.2f), the distribution at $\Delta \varphi=0$ increased significantly. 

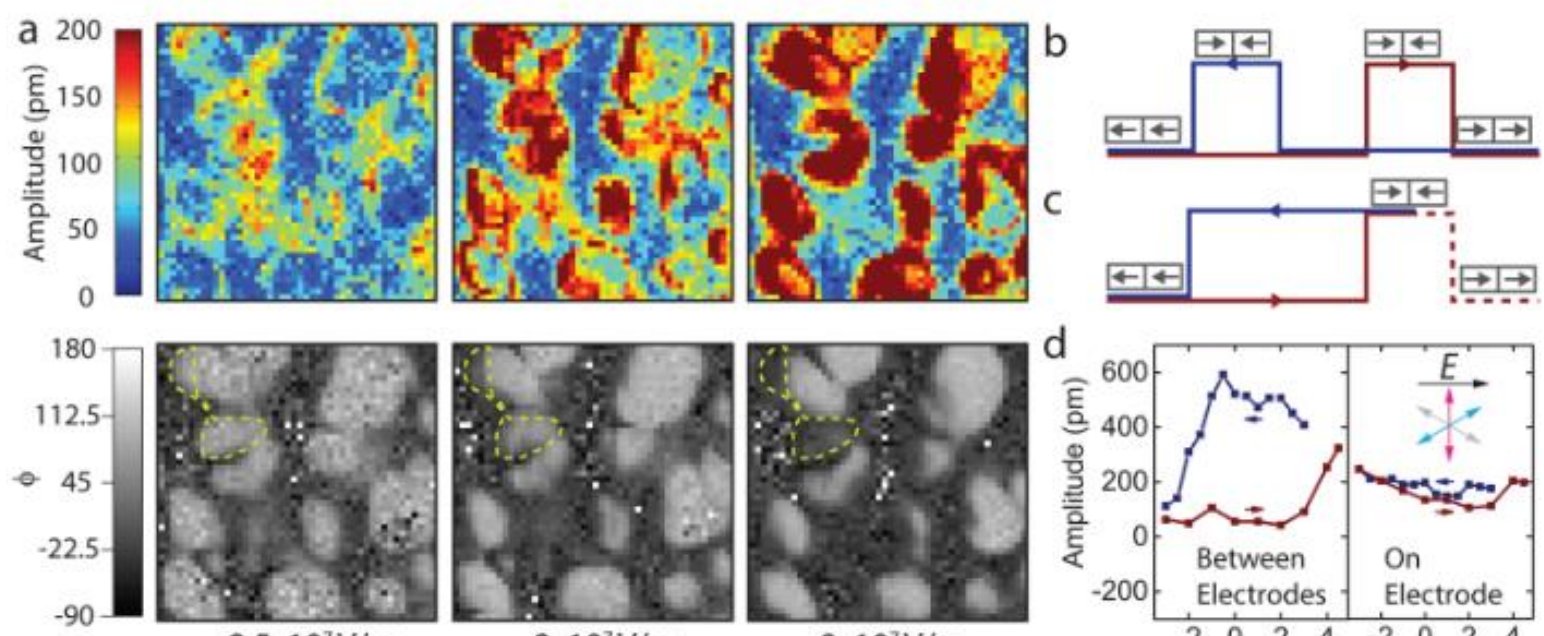

$-2 \times 10^{7} \mathrm{~V} / \mathrm{m}$

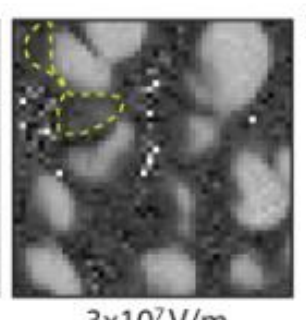

$3 \times 10^{7} \mathrm{~V} / \mathrm{m}$

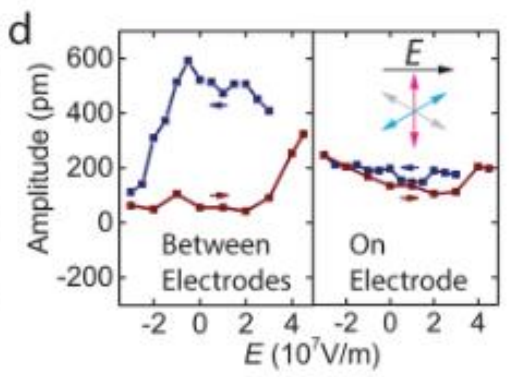

Figure A.3 Ferroelectric switching of $\mathrm{BaCoF}_{4}$ thin film (a) PFM amplitude and phase measured in a $175 \mathrm{~nm} \times 175 \mathrm{~nm}$ area after the application of different in-plane electric fields for $30 \mathrm{~s}$. Areas enclosed by yellow dashed curves indicate regions switched by electric field. (b,c) Illustration of idealized PFM amplitude changes at the boundary between two domains during electric field sweeps shown in $(d)$. Red and blue lines correspond to decreasing and increasing field values, respectively. (b) Maximum amplitude is expected when the two adjacent domains have opposite polarizations, noted by arrows in boxes. (c) When the range of electric field is limited and some domains remain unswitched, the result shows a different PFM amplitude evolution. (d) Experimental evolution of the PFM amplitude at two boundary spots when cycling the in-plane field. (Left) between electrodes where in-plane field is properly applied; (Right) on top of an electrode where no in-plane field is applied. Within the applied field range some domains did not switch, producing a PFM amplitude hysteresis, which highly resembled (c).

To evaluate the ferroelectric properties of the nano-domain structures, an in-plane electric field was applied by biasing the interdigital electrodes. Restricted by leakage, dc electric fields between $-3.1 \times 10^{7} \mathrm{~V} / \mathrm{m}$ and $4.2 \times 10^{7} \mathrm{~V} / \mathrm{m}$ were applied. Figure A.3a shows the PFM images taken in a 175 $\mathrm{nm} \times 175 \mathrm{~nm}$ area after different values of electric field were applied for $30 \mathrm{~s}$ and then turned off. During the measurements, the cantilever was aligned parallel to the dc field orientation. A $\pi$-phase shift was observed only in some regions (e.g., regions highlighted by yellow dash curves) during the field sweep. It is likely that the regions that did not switch contained twin domains with their 
polar axes largely perpendicular to the electric field and other regions that required higher electric field to switch.

Head-to-head domain boundaries were identified from regions with large PFM amplitudes and opposite phases at opposing sides, as discussed above. PFM amplitudes at such locations were tracked as a function of electric field. In principle, because large electric fields aligned the polarizations of adjacent domains, the PFM amplitude was expected to be minimized at both field polarities as illustrated in figure A.3b. However, when one domain required a switching field larger than what was possible in the experiments, the PFM amplitude evolution was expected to be different (figure A.3c). In the experiment two separate spots were studied. One spot (left, figure A.3d) was located between the electrodes, while the other one, as a control experiment, was located on top of a buried electrode (right, figure A.3d) where the in-plane field was zero. Large hysteretic changes of PFM amplitude were only observed in the first case and resembled the situation of a limited domain switching, as illustrated in figure A.3c. The fact that some domains required a much larger switching field than others might originate from a preferred polarization direction in the film or other reasons related to leakage.

$\mathrm{BaCoF}_{4}$ thin films were synthesized for the first time. My collaborators studied the structural and magnetic properties of the films while I was able to utilize a scanning probe with PFM imaging to diagnose nano-scale properties. By obtaining PFM images with different cantilever angles, I was able to map out the polarization orientation of ferroelectric domains. Also I was able to pole individual domains with an applied bias from the scanning probe. This demonstrates another unique use of scanning probes; deforming the topography of a ferroelectric material to measure nanoscale domain orientation, along with directly applying a localized electric field to specific regions. 


\section{Appendix B}

\section{Modulation of LAO/STO Photovoltage Mechanism}

Another example of scanning probe manipulation incorporated into nanotechnology is the modulation of absorption characteristics. Colleagues ${ }^{271}$ and I explored sizable and reconfigurable infrared photovoltage generations at $\mathrm{LaAlO}_{3} / \mathrm{SrTiO}_{3}$ (LAO/STO) interfaces ${ }^{271}$ utilizing the photoexcitations of hot carriers in metal contacts ${ }^{272-276}$. By controlling the local interface metallicity near the contact region using a biased AFM 277-279, two different light-charge conversion mechanisms could be selected. The first of them, originating from the band alignment induced hole filtering effect, was prominent only at low temperatures when the interfacial electron mobility was large. The second one, which required the presence of a two-dimensional electron gas (2DEG), was produced from the photothermoelectric (PTE) effect and significant at room temperature. The relevance of these effects to this dissertation is the probe written 2DEG channels used for thermoelectric generations could be arbitrarily patterned and erased by a biased scanning probe, thus the large PTE effect at the LAO/STO interface was spatially programmable and could be flexibly integrated with other electronic or optical device structures. This AFM 2DEG writing phenomenon was previously discovered by members of my research team ${ }^{280}$.

On LAO/STO samples with 8-unit cell (uc), multiple Ti/Au contact electrodes as shown in figure B.1a were deposited. The majority area of the electrodes (white dashed lines in figure B.1b) were deposited on top of the LAO film, whereas under the tip regions (brown dashed lines in figure B.1b) the LAO film was removed prior to metal deposition by Ar-ion milling to form direct metal contact with the interfacial STO layer. 8 uc LAO/STO normally contains an interfacial 2DEG. We were able to suppress the 2DEG at the beginning using oxygen plasma ${ }^{278}$, then four metallic 2DEG channels were written on the insulated interface by the biased $\mathrm{AFM}^{279}$. These 2DEG channels were optically invisible but could be visualized by KPFM (figure B.1b inset). A continuous wave laser beam $(5 \mathrm{~mW}, 830 \mathrm{~nm})$ focused to a $3 \mu \mathrm{m}$ spot was used for acquiring scanning photovoltage microscopy images. By fixing the laser focal point and scanning the sample, distributions of the photovoltage generation (figure B.1c,e) and optical reflectance (figure B.1b) were mapped simultaneously to allow for accurate positioning of the photovoltage signal generation ${ }^{271}$. 
a
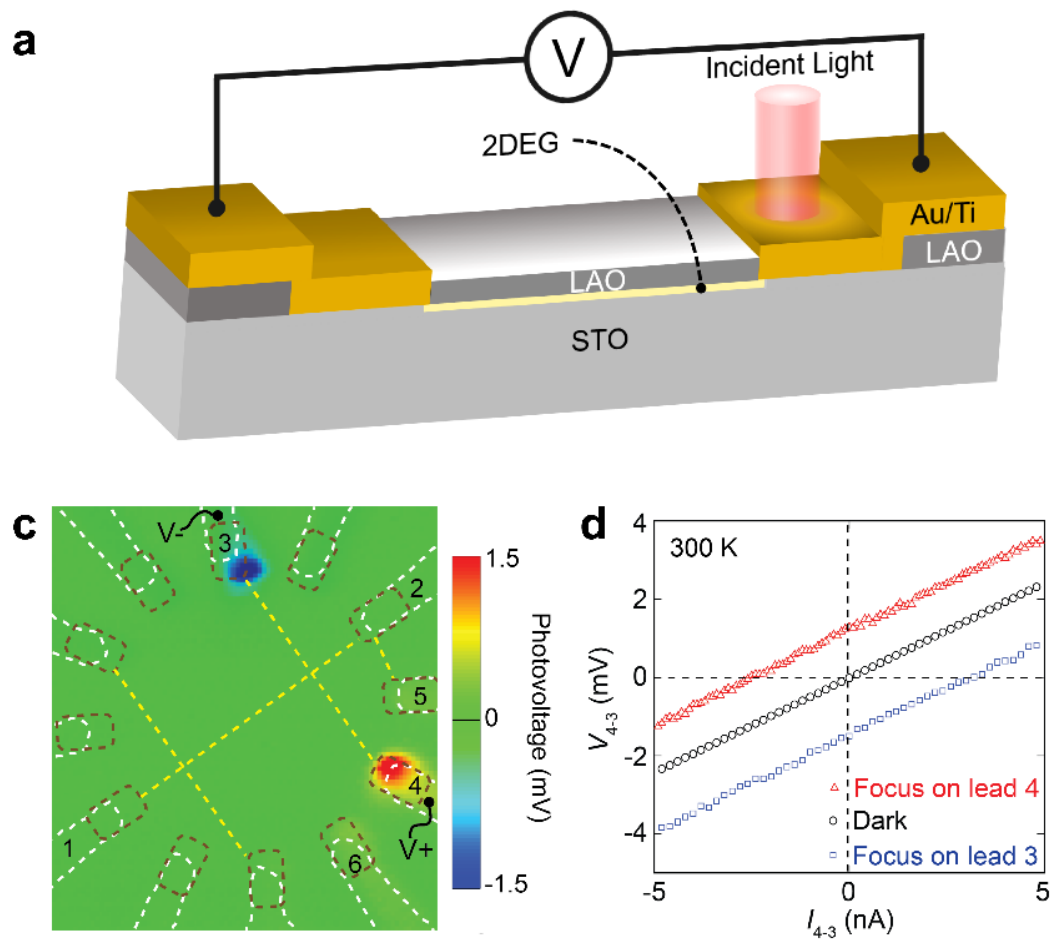

b

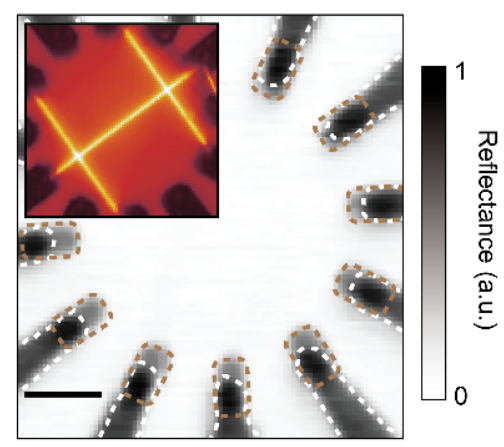

e

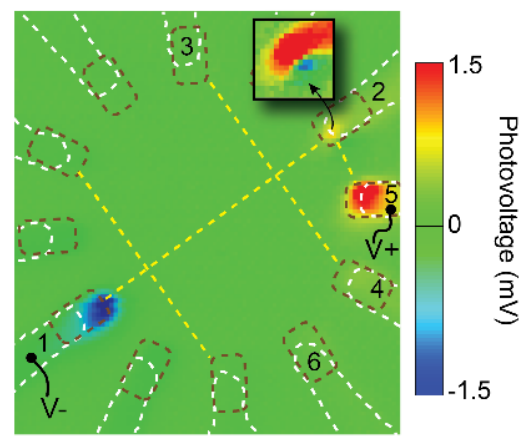

Figure B.1 IR photovoltages across 2DEG channels (a) Schematic of the device and experimental setup. (b) Room temperature scanning optical reflectance images of the $\mathrm{LaAlO}_{3} / \mathrm{SrTiO}_{3}$ device area. The inset KPFM image shows the interface 2DEG channels patterned by c-AFM. (c,e) 830 nm scanning photovoltage images measured between two different pairs of electrodes. Inset in e is an enlarged view of the end of electrode 2 using a color map with smaller photovoltage range. (d) I-V curves between electrode 3 and 4 in dark and when light was focused on the electrodes.

Although the photon energy $(1.5 \mathrm{eV})$ was too low to produce interband excitation in either STO (band gap of $3.2 \mathrm{eV}$ ) or LAO (band gap of $5.6 \mathrm{eV}$ ), a pronounced photovoltage at millivolt level was observed at room temperature across the 2DEG channels when light was focused on their contact electrodes (figure B.1c,e). I-V characteristics measured with and without light were all highly linear (figure B.1d), indicating Ohmic-type metal-oxide contacts instead of Schottkytype junctions ${ }^{281,282}$. The measured photovoltage signal was positive near the anode electrode and negative near the cathode, which means that the photocurrent was carried by either electrons flowing from metal to oxide or holes in the opposite direction.

Interestingly, significant photovoltages could also be generated at metal-oxide junctions without written 2DEG channels (figure B.2). However, the spatial distributions of such signals 
were distinct from the case where the 2DEG was present. When an electrode was not connected by any 2DEG structure (figure B.2b), scanning photovoltage signals were found to be uniformly distributed inside the metal film region that had direct contact with the interfacial STO layers. In contrast, when the electrode was connected to 2DEG, the scanning photovoltage signal became more localized to the electrode corner where the 2DEG channel connected (figure B.2c). In both cases, photovoltages could only be generated when the interior regions of the nontransparent metal contacts were illuminated. Thus, it is more likely that the photoexcitations in the gap-less metal films, rather than in the wide-bandgap oxides, are responsible for the infrared light absorption ${ }^{271}$.
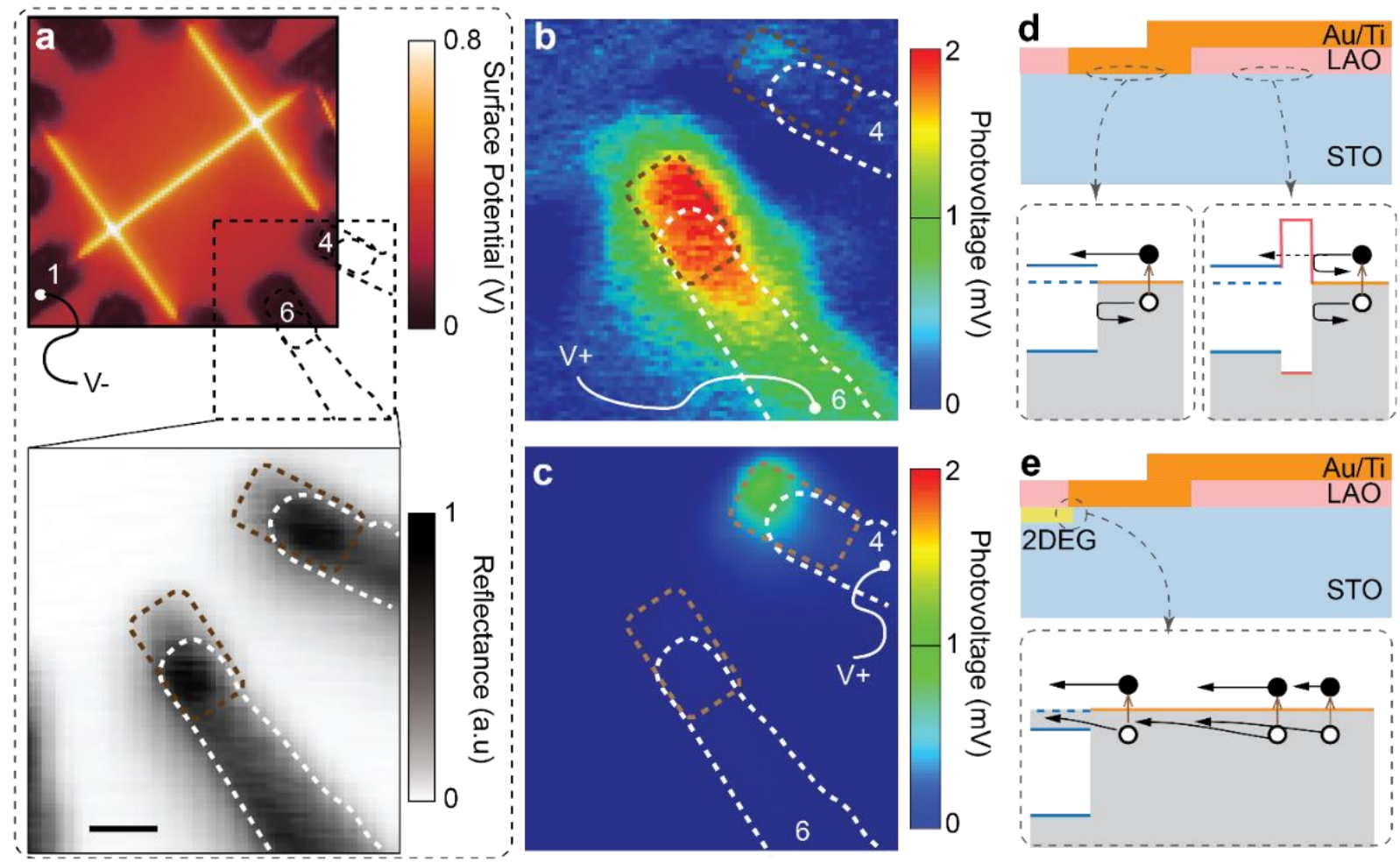

Figure B.2 Photovoltage generation with \& without 2DEG (a) KPFM and scanning optical reflectance images showing the relative positions of $2 D E G$ channels, ohmic contacts to the interface (brown dashed lines), and electrode sections on top of $\mathrm{LaAlO}_{3}$ film (white dashed lines). Scale bar represents $5 \mu \mathrm{m}$. (b,c) With the same cathode electrode as marked in (a), scanning photovoltage images obtained at $6 \mathrm{~K}$ when the anode is connected to two different electrodes: without the 2DEG (b) with the 2DEG channel (c). (d,e) Schematics showing the hot electron excitation in $\mathrm{Au} / \mathrm{Ti}$ electrode and subsequent diffusion of carriers at different junctions. 
In addition, the temperature dependences of the photovoltages detected with and without 2DEG channels were also very different as can be seen in figure B.3b. These distinctions suggested that the two types of photovoltages may have had different origins. The photovoltages generated at electrodes not connected by 2DEG increased significantly below $100 \mathrm{~K}$. They maximized at the lowest temperatures (figure B.3b, black). These behaviors closely follow the trend of the interface electron mobility previously measured ${ }^{279}$. Together with the band alignment at the metal-insulating STO junction as illustrated in figure B.2d, the photovoltage generation without 2DEG could be explained as the following. When photoexcitations occur in the metal electrode, the hot-electrons with energies above the STO conduction band edge can diffuse into the oxide but the holes are blocked by the band gap of STO. Such a charge separation process becomes significant at low temperatures since the enhanced electron mobility tends to increase the electron diffusion length and suppress the electron-hole recombination. In particular, as STO undergoes the cubic-totetragonal structural phase transition at around $105 \mathrm{~K}$, the energy degeneracy of the three $t_{2 \mathrm{~g}}$ subbands is lifted ${ }^{283}$. The increased carrier occupation in the lighter $d_{x y}$ band as comparing to the heavier $d_{x z}, d_{y z}$ bands can induce a pronounced carrier mobility enhancement in $\mathrm{STO}^{279}$. Such effect is likely responsible for the photovoltage upturn observed at around $100 \mathrm{~K}$ (figure B.3b). The metal-oxide band alignment induced charge separation mechanism also explains why scanning photovoltage signals measured in electrode regions that were deposited on top of LAO (figure B.2b, regions enclosed within white dashed lines) were much weaker than the signal detected in electrode regions in direct contact with STO (figure B.2b, regions enclosed within brown dashed lines). When there was a thin LAO layer between the contact metal and STO, the larger bandgap of LAO forms an additional energy barrier that the hot electrons need to overcome in order to diffuse away from the holes trapped inside the metal (figure B.2d). For the photoexcited electrons with energy above STO conduction band edge but below LAO conduction band edge, their only diffusion pathway was through tunneling. Consequently, the lower rate of electron diffusion at the metal/oxide interface weakened the charge separation and reduced the photovoltage generation ${ }^{271}$. However, such charge separation mechanism cannot explain the photovoltage generation at electrodes connected by 2DEG channels. As shown in figure B.2e, the Fermi level in the 2DEG region is above the conduction band. In that case there is no longer a barrier from the wide bandgap oxides to prevent hole diffusion from electrode to STO. 
a

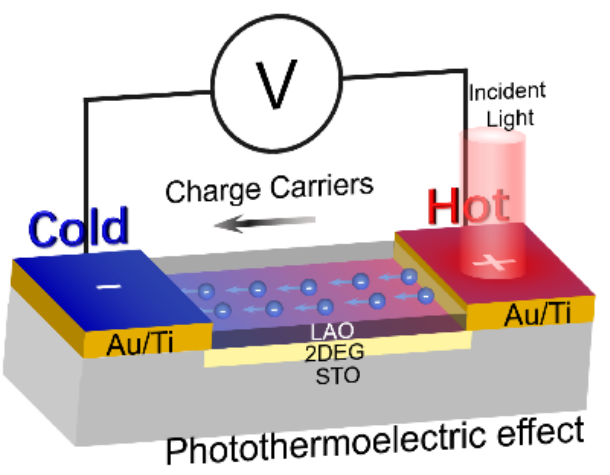

C

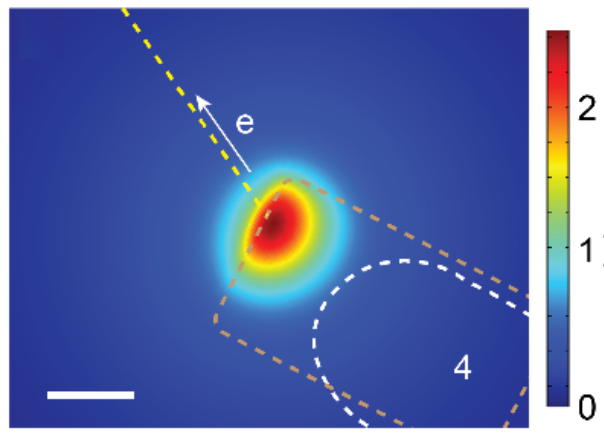

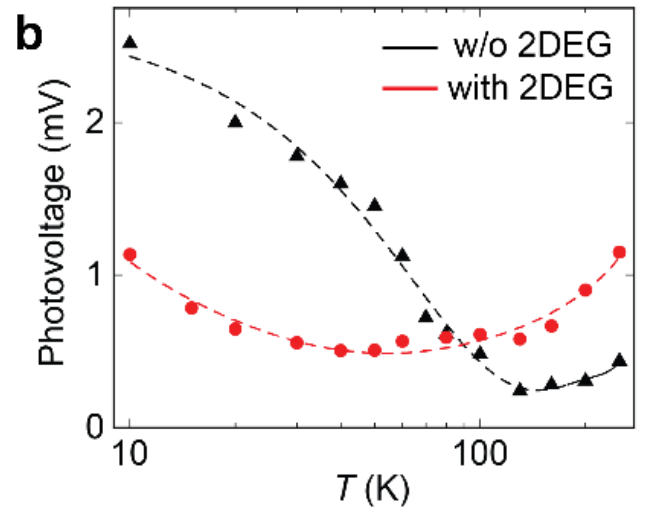

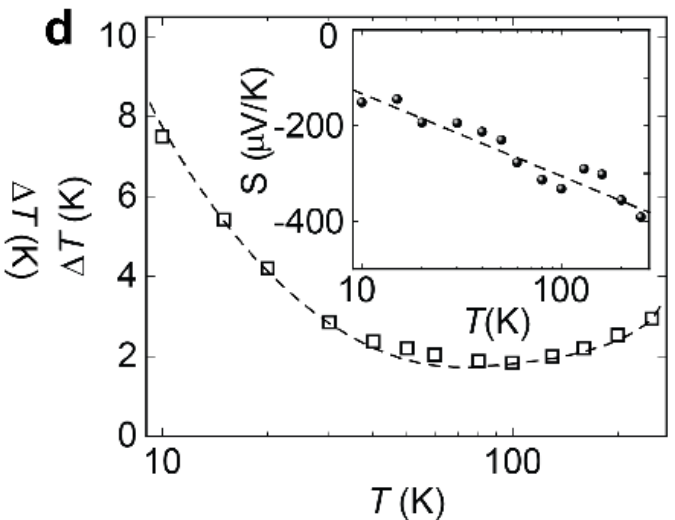

Figure B.3 Temperature dependences of the photovoltage at LAO/STO interfaces (a) Schematic of infrared PTE voltage generation by 2DEG channel (b) Temperature dependences of the photovoltages detected at interface contacts with and without 2DEG channel connected. (c) Simulated distribution of temperature increase caused by laser heating at room temperature when light is focused at the electrode-2DEG junction. The model considers a measured electrode reflectance of $96 \%$ and a literature reported extinction coefficient of $5.2^{290}$. Scale bar represents $3 \mu \mathrm{m}$. (d) Light induced temperature increases at the end of the 2DEG channel as a function of the background sample temperature. Inset: Temperature dependent Seebeck coefficient in the 2DEG channel calculated from the measured photovoltages and simulated temperature changes.

The thermoelectric effect accounts for the photovoltage generation in the 2DEG case. The thermoelectric effect can be greatly enhanced in material systems with reduced dimensionalities ${ }^{284,285}$. As the photoexcited hot electrons in the electrode relaxed back to their ground state, the energy dissipation via electron-phonon and electron-electron scattering produced a local heating effect. Based on a FEM simulation (figure B.3c), a local heating of $\Delta T \sim 2 \mathrm{~K}$ could 
be produced at room temperature with the laser focused on the metal electrode. While such photoheating is rather weak, the giant Seebeck coefficient $(S)$ in the interface 2DEG channel ${ }^{286,287}$ allows for efficient photovoltage generation through thermoelectric heat conversion ${ }^{273,288,289}$ ( $\Delta V=-S \Delta T$ ) (figure B.3a). Measured values of the generated photovoltage ( $\Delta V)$ vs temperature are shown in figure B.3b. Combined with the FEM simulation for the change in temperature due to photoheating ( $\Delta T$ in figure B.3d), the temperature dependent Seebeck coefficient (S) could be extracted as the ratio $-\Delta V / \Delta T$ from the thermoelectric heat conversion $(\Delta V=-S \Delta T$ ) (figure B.3d, inset). The extracted curve was highly consistent with the literature reported conventional thermoelectric characterization result ${ }^{286}$. The great match further supported the PTE nature of the large photovoltages measured across 2DEG channels.

My colleagues and I were able to demonstrate substantial infrared photovoltage generations at LAO/STO interfaces with modulation capability. In one case, hot electrons generated in $\mathrm{Au} / \mathrm{Ti}$ electrodes could diffuse into the oxides separating electron-hole pairs. Then by utilizing scanning probe lithography yet again, I was able to induce, erase, and manipulate 2DEG channels to create a photothermal generation because of the giant Seebeck coefficient. Control over the 2DEG channels provide the opportunitiy to take advantage of the Seebeck coefficient for spatially programmable photovoltaics to be incorporated into future optoelectronic devices. LAO/STO is one more platform example where scanning probe lithography can be used for nano-scale manipulation to integrate reprogrammable functions into future device architecture. 


\section{References}

1 Predeep, P. Optoelectronics: Materials and Techniques. (BoD-Books on Demand, 2011).

2 Parker, M. A. Physics of optoelectronics. (CRC Press, 2018).

3 Monticone, F. \& Alu, A. Metamaterial, plasmonic and nanophotonic devices. Reports on Progress in Physics 80, 036401 (2017).

4 Saleh, B. E. \& Teich, M. C. Fundamentals of photonics. (john Wiley \& sons, 2019).

5 Yu, N. \& Capasso, F. Flat optics with designer metasurfaces. Nature materials 13, 139 (2014).

6 contributors, W. Metamaterial, <https://en.wikipedia.org/wiki/Metamaterial>

7 Yongmin Liu, X. Z. Metamaterial, 〈https://www.britannica.com/topic/metamaterial $>$ (2016).

8 Shelby, R. A., Smith, D. R. \& Schultz, S. Experimental verification of a negative index of refraction. science 292, 77-79 (2001).

9 Zhang, S. et al. Experimental demonstration of near-infrared negative-index metamaterials. Physical review letters 95, 137404 (2005).

10 Soukoulis, C. M., Linden, S. \& Wegener, M. Negative refractive index at optical wavelengths. Science 315, 47-49 (2007).

11 Pendry, J. B. Negative refraction makes a perfect lens. Physical review letters 85, 3966 (2000).

12 Zhang, X. \& Liu, Z. Superlenses to overcome the diffraction limit. Nature materials 7, 435-441 (2008).

13 Landy, N. I., Sajuyigbe, S., Mock, J. J., Smith, D. R. \& Padilla, W. J. Perfect metamaterial absorber. Physical review letters 100, 207402 (2008).

14 Watts, C. M., Liu, X. \& Padilla, W. J. Metamaterial electromagnetic wave absorbers. Advanced materials 24, OP98-OP120 (2012).

15 Lee, Y. P., Rhee, J. Y., Yoo, Y. J. \& Kim, K. W. Metamaterials for perfect absorption. Vol. 236 (Springer, 2016).

16 Fernandez-Corbaton, I., Fruhnert, M. \& Rockstuhl, C. Objects of maximum electromagnetic chirality. Physical Review X 6, 031013 (2016).

17 Kauranen, M. \& Zayats, A. V. Nonlinear plasmonics. Nature photonics 6, 737 (2012).

18 Lee, J. et al. Giant nonlinear response from plasmonic metasurfaces coupled to intersubband transitions. Nature 511, 65-69 (2014).

19 Huygens, C. Traité de la lumiere (Leiden, 1690). Oeuvres completes XIX 1737 (1912).

20 Glybovski, S. B., Tretyakov, S. A., Belov, P. A., Kivshar, Y. S. \& Simovski, C. R. Metasurfaces: From microwaves to visible. Physics reports 634, 1-72 (2016).

21 Kildishev, A. V., Boltasseva, A. \& Shalaev, V. M. Planar photonics with metasurfaces. Science 339, 1232009 (2013).

22 Zheludev, N. I. \& Kivshar, Y. S. From metamaterials to metadevices. Nature materials 11, 917-924 (2012).

23 Hand, T. \& Cummer, S. Characterization of tunable metamaterial elements using MEMS switches. IEEE Antennas and Wireless Propagation Letters 6, 401-404 (2007).

24 Ou, J.-Y., Plum, E., Jiang, L. \& Zheludev, N. I. Reconfigurable photonic metamaterials. Nano letters 11, 2142-2144 (2011). 
25 Zheludev, N. I. \& Kivshar, Y. S. From metamaterials to metadevices. Nature materials 11, 917 (2012).

26 Zhao, Q. et al. Electrically tunable negative permeability metamaterials based on nematic liquid crystals. Applied physics letters 90, 011112 (2007).

27 Werner, D. H., Kwon, D.-H., Khoo, I.-C., Kildishev, A. V. \& Shalaev, V. M. Liquid crystal clad near-infrared metamaterials with tunable negative-zero-positive refractive indices. Optics Express 15, 3342-3347 (2007).

28 Gil, M. et al. Electrically tunable split-ring resonators at microwave frequencies based on barium-strontium-titanate thick films. Electronics letters 45, 417-418 (2009).

29 Papasimakis, N. et al. Graphene in a photonic metamaterial. Optics express 18, 8353-8359 (2010).

30 Yan, H. et al. Tunable infrared plasmonic devices using graphene/insulator stacks. Nature nanotechnology 7, 330-334 (2012).

31 Yao, Y. et al. Electrically tunable metasurface perfect absorbers for ultrathin mid-infrared optical modulators. Nano letters 14, 6526-6532 (2014).

32 Goldflam, M. et al. Reconfigurable gradient index using VO2 memory metamaterials. Applied Physics Letters 99, 044103 (2011).

33 Dicken, M. J. et al. Frequency tunable near-infrared metamaterials based on VO 2 phase transition. Optics express 17, 18330-18339 (2009).

$34 \mathrm{Li}, \mathrm{Z}$. et al. Correlated Perovskites as a New Platform for Super-Broadband-Tunable Photonics. Advanced Materials 28, 9117-9125 (2016).

35 Gholipour, B., Zhang, J., MacDonald, K. F., Hewak, D. W. \& Zheludev, N. I. An alloptical, non-volatile, bidirectional, phase-change meta-switch. Advanced materials 25, 3050-3054 (2013).

36 Michel, A.-K. U. et al. Reversible optical switching of infrared antenna resonances with ultrathin phase-change layers using femtosecond laser pulses. Acs Photonics 1, 833-839 (2014).

37 Wuttig, M. \& Yamada, N. Phase-change materials for rewriteable data storage. Nature materials 6, 824-832 (2007).

38 Raeis-Hosseini, N. \& Rho, J. Metasurfaces based on phase-change material as a reconfigurable platform for multifunctional devices. Materials 10, 1046 (2017).

39 Cueff, S. et al. Dynamic control of light emission faster than the lifetime limit using VO 2 phase-change. Nature communications 6, 8636 (2015).

40 Lencer, D. et al. A map for phase-change materials. Nature materials 7, 972 (2008).

41 Xu, G., Jin, P., Tazawa, M. \& Yoshimura, K. Tailoring of luminous transmittance upon switching for thermochromic VO2 films by thickness control. Japanese journal of applied physics 43, 186 (2004).

42 Currie, M., Mastro, M. A. \& Wheeler, V. D. Characterizing the tunable refractive index of vanadium dioxide. Optical Materials Express 7, 1697-1707 (2017).

43 Shportko, K. et al. Resonant bonding in crystalline phase-change materials. Nature materials 7, 653 (2008).

44 Qazilbash, M. M. et al. Mott Transition in $\mathrm{VO}_{2}$ Revealed by Infrared Spectroscopy and Nano-Imaging. Science 318, 1750-1753, doi:10.1126/science.1150124 (2007).

45 Kats, M. A. et al. Thermal tuning of mid-infrared plasmonic antenna arrays using a phase change material. Optics letters 38, 368-370 (2013). 
46 Makino, K., Tominaga, J. \& Hase, M. Ultrafast optical manipulation of atomic arrangements in chalcogenide alloy memory materials. Optics express 19, 1260-1270 (2011).

47 Nag, J. et al. in Physics and Simulation of Optoelectronic Devices XVIII. 759710 (International Society for Optics and Photonics).

48 Loke, D. et al. Breaking the speed limits of phase-change memory. Science 336, 15661569 (2012).

49 Radu, I. P. et al. Switching mechanism in two-terminal vanadium dioxide devices. Nanotechnology 26, 165202 (2015).

50 Zhang, H.-T. et al. Wafer-scale growth of $\mathrm{VO}_{2}$ thin films using a combinatorial approach. Nat Commun 6, doi:10.1038/ncomms9475 (2015).

51 Martens, K., Aetukuri, N., Jeong, J., Samant, M. G. \& Parkin, S. S. Improved metalinsulator-transition characteristics of ultrathin VO2 epitaxial films by optimized surface preparation of rutile TiO2 substrates. Applied Physics Letters 104, 081918 (2014).

52 Ruzmetov, D., Zawilski, K. T., Narayanamurti, V. \& Ramanathan, S. Structure-functional property relationships in rf-sputtered vanadium dioxide thin films. Journal of Applied Physics 102, 113715 (2007).

53 Fan, L. et al. Growth and phase transition characteristics of pure M-phase VO2 epitaxial film prepared by oxide molecular beam epitaxy. Applied Physics Letters 103, 131914 (2013).

54 Ross, U., Lotnyk, A., Thelander, E. \& Rauschenbach, B. Microstructure evolution in pulsed laser deposited epitaxial Ge-Sb-Te chalcogenide thin films. Journal of Alloys and Compounds 676, 582-590 (2016).

55 Momand, J. et al. Atomic stacking and van-der-Waals bonding in GeTe-Sb 2 Te 3 superlattices. Journal of Materials Research 31, 3115-3124 (2016).

56 Ratajczak, A., von der Ahe, M., Du, H., Mussler, G. \& Grützmacher, D. Metal organic vapor phase epitaxy of $\$ \$$ hbox $\left.\{\mathrm{Ge}\}_{-}\{1\} \backslash \mathrm{hbox}\{\mathrm{Sb}\}_{-}\{2\} \backslash \mathrm{hbox}\{\mathrm{Te}\}\right\}_{-}\{4\} \$ \$$ thin films on Si (111) substrate. Applied Physics A 125, 163 (2019).

57 Wuttig, M., Bhaskaran, H. \& Taubner, T. Phase-change materials for non-volatile photonic applications. Nature Photonics 11, 465 (2017).

58 Wang, Q. et al. Optically reconfigurable metasurfaces and photonic devices based on phase change materials. Nature Photonics 10, 60 (2016).

59 Raoux, S. in Phase Change Materials 99-124 (Springer, 2009).

60 Kolobov, A. V., Fons, P., Tominaga, J. \& Uruga, T. Why DVDs work the way they do: The nanometer-scale mechanism of phase change in $\mathrm{Ge}-\mathrm{Sb}-\mathrm{Te}$ alloys. Journal of noncrystalline solids 352, 1612-1615 (2006).

61 Hosseini, P., Wright, C. D. \& Bhaskaran, H. An optoelectronic framework enabled by lowdimensional phase-change films. Nature 511, 206-211 (2014).

62 Jeong, J. et al. Suppression of Metal-Insulator Transition in $\mathrm{VO}_{2}$ by Electric Field-Induced Oxygen Vacancy Formation. Science 339, 1402-1405, doi:10.1126/science.1230512 (2013).

63 Jeong, J. et al. Giant reversible, facet-dependent, structural changes in a correlated-electron insulator induced by ionic liquid gating. Proceedings of the National Academy of Sciences 112, 1013-1018, doi:10.1073/pnas.1419051112 (2015). 
64 Rensberg, J. et al. Active optical metasurfaces based on defect-engineered phase-transition materials. Nano letters 16, 1050-1055 (2016).

65 Bimberg, D. et al. in Optoelectronic Integrated Circuits XIV. 82650E (International Society for Optics and Photonics).

66 Berglund, C. N. \& Guggenheim, H. J. Electronic Properties of $\mathrm{VO}_{2}$ near the Semiconductor-Metal Transition. Physical Review 185, 1022-1033 (1969).

67 Cavalleri, A., Dekorsy, T., Chong, H. H. W., Kieffer, J. C. \& Schoenlein, R. W. Evidence for a structurally-driven insulator-to-metal transition in $\mathrm{VO}_{2}$ : A view from the ultrafast timescale. Physical Review B 70, 161102 (2004).

68 Basov, D. N., Averitt, R. D., Van Der Marel, D., Dressel, M. \& Haule, K. Electrodynamics of correlated electron materials. Reviews of Modern Physics 83, 471 (2011).

69 Ramanathan, S. Thin film metal-oxides. Harvard University: Springer New York Dordrecht Heidelberg London (2010).

70 Morin, F. Oxides which show a metal-to-insulator transition at the Neel temperature. Physical review letters 3, 34 (1959).

71 Kachi, S., Kosuge, K. \& Okinaka, H. Metal-insulator transition in VnO2n- 1. Journal of Solid State Chemistry 6, 258-270 (1973).

72 Rosevear, W. \& Paul, W. Hall Effect in V O 2 near the Semiconductor-to-Metal Transition. Physical Review B 7, 2109 (1973).

73 Schwingenschlögl, U. \& Eyert, V. The vanadium Magnéli phases VnO2n-1. Annalen der physik 13, 475-510 (2004).

74 McWhan, D., Marezio, M., Remeika, J. \& Dernier, P. X-ray diffraction study of metallic V O 2. Physical Review B 10, 490 (1974).

75 Zylbersztejn, A. \& Mott, N. F. Metal-insulator transition in vanadium dioxide. Physical Review B 11, 4383 (1975).

76 Goodenough, J. B. Band structure of transition metals and their alloys. Physical Review 120, 67 (1960).

77 Kucharczyk, D. \& Niklewski, T. Accurate X-ray determination of the lattice parameters and the thermal expansion coefficients of VO2 near the transition temperature. Journal of Applied Crystallography 12, 370-373 (1979).

78 Eyert, V. The metal-insulator transitions of VO2: A band theoretical approach. Annalen der Physik 11, 650-704 (2002).

$79 \mathrm{Wu}, \mathrm{Y}$. et al. Depressed transition temperature of $\mathrm{W} \times \mathrm{V} 1-\mathrm{x} \mathrm{O} 2$ : mechanistic insights from the X-ray absorption fine structure (XAFS) spectroscopy. Physical Chemistry Chemical Physics 16, 17705-17714 (2014).

80 Goodenough, J. B. The two components of the crystallographic transition in $\mathrm{VO}_{2}$. Journal of Solid State Chemistry 3, 490-500, doi:http://dx.doi.org/10.1016/0022-4596(71)90091-0 (1971).

81 Aetukuri, N. B. et al. Control of the metal-insulator transition in vanadium dioxide by modifying orbital occupancy. Nat Phys 9, 661-666, doi:10.1038/nphys2733http://www.nature.com/nphys/journal/v9/n10/abs/nphys2733.html \#supplementary-information (2013).

82 Chen, S. et al. The Dynamic Phase Transition Modulation of Ion-Liquid Gating VO2 Thin Film: Formation, Diffusion, and Recovery of Oxygen Vacancies. Advanced Functional Materials 26, 3532-3541, doi:10.1002/adfm.201505399 (2016). 
83 Haverkort, M. W. et al. Orbital-Assisted Metal-Insulator Transition in $\mathrm{VO}_{2}$. Physical Review Letters 95, 196404 (2005).

84 Metal-Oxides, T. F. (Ramanathan, S., Ed, 2010).

85 Kim, H.-T. et al. Monoclinic and correlated metal phase in VO 2 as evidence of the Mott transition: coherent phonon analysis. Physical review letters 97, 266401 (2006).

86 CaoJ et al. Strain engineering and one-dimensional organization of metal-insulator domains in single-crystal vanadium dioxide beams. Nat Nano 4, 732-737, doi:http://www.nature.com/nnano/journal/v4/n11/suppinfo/nnano.2009.266_S1.html (2009).

87 Schrecongost, D. et al. On-Demand Nanoscale Manipulations of Correlated Oxide Phases. Advanced Functional Materials, 1905585 (2019).

88 Wei, J., Ji, H., Guo, W., Nevidomskyy, A. H. \& Natelson, D. Hydrogen stabilization of metallic vanadium dioxide in single-crystal nanobeams. Nat Nano 7, 357-362, doi:http://www.nature.com/nnano/journal/v7/n6/abs/nnano.2012.70.html\#supplementaryinformation (2012).

89 Jaffari, G. H. \& Mahmood, W. Investigation of phase evolution and control over phase transformation temperature and thermal hysteresis using stoichiometry and co-doping in VO2 thin films. AIP Advances 7, 115312 (2017).

90 Wang, S. et al. Recent progress in VO2 smart coatings: Strategies to improve the thermochromic properties. Progress in Materials Science 81, 1-54 (2016).

$91 \mathrm{Wu}, \mathrm{Y}$. et al. Decoupling the lattice distortion and charge doping effects on the phase transition behavior of VO 2 by titanium (Ti 4+) doping. Scientific reports $\mathbf{5}, 9328$ (2015).

92 Krammer, A. et al. Elevated transition temperature in Ge doped VO2 thin films. Journal of Applied Physics 122, 045304 (2017).

93 Piccirillo, C., Binions, R. \& Parkin, I. P. Nb-Doped VO2 Thin Films Prepared by AerosolAssisted Chemical Vapour Deposition. European Journal of Inorganic Chemistry 2007, 4050-4055 (2007).

94 Hanlon, T., Coath, J. \& Richardson, M. Molybdenum-doped vanadium dioxide coatings on glass produced by the aqueous sol-gel method. Thin Solid Films 436, 269-272 (2003).

95 Mlyuka, N., Niklasson, G. \& Granqvist, C.-G. Mg doping of thermochromic VO 2 films enhances the optical transmittance and decreases the metal-insulator transition temperature. Applied physics letters 95, 171909 (2009).

96 Brown, B. et al. Electrical and optical characterization of the metal-insulator transition temperature in Cr-doped VO2 thin films. Journal of Applied Physics 113, 173704 (2013).

97 Brückner, W., Gerlach, U., Brückner, H. P., Moldenhauer, W. \& Oppermann, H. Influence of nonstoichiometry on the phase transitions in $\mathrm{Ga}-$, Al-, and Fe-doped VO2. physica status solidi (a) 42, 295-303 (1977).

98 Nakano, M. et al. Collective bulk carrier delocalization driven by electrostatic surface charge accumulation. Nature 487, 459-462, doi:http://www.nature.com/nature/journal/v487/n7408/abs/nature11296.html\#supplement ary-information (2012).

99 Karel, J. et al. Distinct electronic structure of the electrolyte gate-induced conducting phase in vanadium dioxide revealed by high-energy photoelectron spectroscopy. ACS nano $\mathbf{8}$, 5784-5789 (2014). 
100 Zhang, H.-T. et al. Imprinting of Local Metallic States into $\mathrm{VO}_{2}$ with Ultraviolet Light. Advanced Functional Materials 26, 6612-6618, doi:10.1002/adfm.201601890 (2016).

101 Ladd, L. A. \& Paul, W. Optical and transport properties of high quality crystals of V2O4 near the metallic transition temperature. Solid State Communications 7, 425-428 (1969).

102 Pelleg, J. Mechanical properties of materials. Vol. 190 (Springer Science \& Business Media, 2012).

103 Benda, V., Grant, D. A. \& Gowar, J. Discrete and Integrated Power Semiconductor Devices: Theory and Applications. (John Wiley \& Sons, 1999).

104 Passarello, D. et al. Evidence for Ionic Liquid Gate-Induced Metallization of Vanadium Dioxide Bars over Micron Length Scales. Nano letters 17, 2796-2801 (2017).

105 Appavoo, K. et al. Role of defects in the phase transition of VO2 nanoparticles probed by plasmon resonance spectroscopy. Nano letters 12, 780-786 (2012).

106 Mellan, T. A. \& Grau-Crespo, R. Density functional theory study of rutile VO2 surfaces. The Journal of chemical physics 137, 154706 (2012).

107 Hutter, J. L. \& Bechhoefer, J. Calibration of atomic-force microscope tips. Review of Scientific Instruments 64, 1868-1873 (1993).

108 Hrouzek, M. Atomic Force Microscopy, modeling, estimation and control, PhD thesis, Université Joseph Fourier, (2007).

109 Meyer, E. Atomic force microscopy. Progress in surface science 41, 3-49 (1992).

110 Garcia, R. \& San Paulo, A. Attractive and repulsive tip-sample interaction regimes in tapping-mode atomic force microscopy. Physical Review B 60, 4961 (1999).

111 Garcia, R., Knoll, A. W. \& Riedo, E. Advanced scanning probe lithography. Nature nanotechnology 9, 577 (2014).

112 Acikgoz, C., Hempenius, M. A., Huskens, J. \& Vancso, G. J. Polymers in conventional and alternative lithography for the fabrication of nanostructures. European Polymer Journal 47, 2033-2052 (2011).

113 Zheng, Y., Wang, H., Hou, S. \& Xia, D. Lithographically defined graphene patterns. Advanced Materials Technologies 2, 1600237 (2017).

114 Fan, J. et al. Investigation of the influence on graphene by using electron-beam and photolithography. Solid state communications 151, 1574-1578 (2011).

$115 \mathrm{Li}$, L. et al. Black phosphorus field-effect transistors. Nature nanotechnology 9, 372 (2014).

$116 \mathrm{Xu}, \mathrm{W} . \&$ Lee, T.-W. Recent progress in fabrication techniques of graphene nanoribbons. Materials Horizons 3, 186-207 (2016).

117 Han, P. et al. Highly sensitive MoS2 photodetectors with graphene contacts. Nanotechnology 29, 20LT01 (2018).

118 Rani, E. \& Wong, L. S. High-Resolution Scanning Probe Nanolithography of 2D Materials: Novel Nanostructures. Advanced Materials Technologies 4, 1900181 (2019).

119 Salaita, K., Wang, Y. \& Mirkin, C. A. Applications of dip-pen nanolithography. Nature nanotechnology 2, 145 (2007).

120 Martínez, R. V., Martínez, J. \& Garcia, R. Silicon nanowire circuits fabricated by AFM oxidation nanolithography. Nanotechnology 21, 245301 (2010).

121 Ryu Cho, Y. K. et al. Sub-10 nanometer feature size in silicon using thermal scanning probe lithography. ACS nano 11, 11890-11897 (2017). 
122 Wang, D. et al. Direct writing and characterization of poly (p-phenylene vinylene) nanostructures. Applied Physics Letters 95, 233108 (2009).

123 Fenwick, O. et al. Thermochemical nanopatterning of organic semiconductors. Nature nanotechnology 4, 664-668 (2009).

124 Felts, J. R., Onses, M. S., Rogers, J. A. \& King, W. P. Nanometer Scale Alignment of Block-Copolymer Domains by Means of a Scanning Probe Tip. Advanced Materials 26, 2999-3002 (2014).

125 Martínez, R. V. et al. Large-scale Nanopatterning of Single Proteins used as Carriers of Magnetic Nanoparticles. Advanced Materials 22, 588-591 (2010).

126 Kim, S. et al. Direct Fabrication of Arbitrary-Shaped Ferroelectric Nanostructures on Plastic, Glass, and Silicon Substrates. Advanced Materials 23, 3786-3790 (2011).

127 Yang, M. et al. Room temperature ferroelectricity in fluoroperovskite thin films. Scientific reports 7, 1-9 (2017).

128 Giesbers, A. et al. Nanolithography and manipulation of graphene using an atomic force microscope. Solid State Communications 147, 366-369 (2008).

129 Weng, L., Zhang, L., Chen, Y. P. \& Rokhinson, L. P. Atomic force microscope local oxidation nanolithography of graphene. Applied Physics Letters 93, 093107 (2008).

130 Byun, I.-S. et al. Nanoscale lithography on monolayer graphene using hydrogenation and oxidation. ACS nano 5, 6417-6424 (2011).

131 Wei, Z. et al. Nanoscale tunable reduction of graphene oxide for graphene electronics. Science 328, 1373-1376 (2010).

$132 \mathrm{Lu}, \mathrm{G}$. et al. Nanolithography of single-layer graphene oxide films by atomic force microscopy. Langmuir 26, 6164-6166 (2010).

133 Liu, X. et al. Scanning Probe Nanopatterning and Layer-by-Layer Thinning of Black Phosphorus. Advanced Materials 29, 1604121 (2017).

134 Fernandes, T. F. et al. Robust nanofabrication of monolayer MoS2 islands with strong photoluminescence enhancement via local anodic oxidation. 2D Materials 5, 025018 (2018).

135 Donarelli, M. et al. Few layered MoS 2 lithography with an AFM tip: description of the technique and nanospectroscopy investigations. Nanoscale 7, 11453-11459 (2015).

136 Dago, A. I., Ryu, Y. K. \& Garcia, R. Sub-20 nm patterning of thin layer WSe2 by scanning probe lithography. Applied Physics Letters 109, 163103 (2016).

137 Girard, P. Electrostatic force microscopy: principles and some applications to semiconductors. Nanotechnology 12, 485 (2001).

138 Melitz, W., Shen, J., Kummel, A. C. \& Lee, S. Kelvin probe force microscopy and its application. Surface science reports 66, 1-27 (2011).

139 Nonnenmacher, M., o'Boyle, M. \& Wickramasinghe, H. K. Kelvin probe force microscopy. Applied physics letters 58, 2921-2923 (1991).

140 Kelvin, L. V. Contact electricity of metals. The London, Edinburgh, and Dublin Philosophical Magazine and Journal of Science 46, 82-120 (1898).

141 Zerweck, U., Loppacher, C., Otto, T., Grafström, S. \& Eng, L. M. Accuracy and resolution limits of Kelvin probe force microscopy. Physical Review B 71, 125424 (2005).

142 Garrett, J. L. \& Munday, J. N. Fast, high-resolution surface potential measurements in air with heterodyne Kelvin probe force microscopy. Nanotechnology 27, 245705 (2016). 
143 Sugawara, Y. et al. High potential sensitivity in heterodyne amplitude-modulation Kelvin probe force microscopy. Applied Physics Letters 100, 223104 (2012).

144 Ocelic, N. Quantitative near-field phonon-polariton spectroscopy, Technische Universität München, (2007).

145 Pauling, L. Metal-metal bond lengths in complexes of transition metals. Proceedings of the National Academy of Sciences 73, 4290-4293 (1976).

146 Danzebrink, H.-U., Kazantsev, D., Dal Savio, C., Pierz, K. \& Güttler, B. Optical microscope with SNOM option for micro-and nanoanalytical investigations at low temperatures. Applied Physics A 76, 889-892 (2003).

147 Greffet, J.-J. \& Carminati, R. Image formation in near-field optics. Progress in surface science 56, 133-237 (1997).

148 Xiao, S., Zhu, X., Li, B.-H. \& Mortensen, N. A. Graphene-plasmon polaritons: From fundamental properties to potential applications. Frontiers of Physics 11, 117801 (2016).

149 Law, S., Yu, L. \& Wasserman, D. Epitaxial growth of engineered metals for mid-infrared plasmonics. Journal of Vacuum Science \& Technology B, Nanotechnology and Microelectronics: Materials, Processing, Measurement, and Phenomena 31, 03C121 (2013).

150 Sachet, E., Losego, M. D., Guske, J., Franzen, S. \& Maria, J.-P. Mid-infrared surface plasmon resonance in zinc oxide semiconductor thin films. Applied Physics Letters 102, 051111 (2013).

151 Huber, A., Ocelic, N., Kazantsev, D. \& Hillenbrand, R. Near-field imaging of mid-infrared surface phonon polariton propagation. Applied physics letters 87, 081103 (2005).

152 Ghamsari, B. G., Xu, X. G., Gilburd, L., Walker, G. C. \& Berini, P. Mid-infrared surface phonon polaritons in boron-nitride nanotubes. Journal of Optics 16, 114008 (2014).

153 Sumikura, H. et al. Highly confined and switchable mid-infrared surface phonon polariton resonances of planar circular cavities with a phase change material. Nano letters 19, 25492554 (2019).

154 Zangwill, A. Modern electrodynamics. (Cambridge University Press, 2013).

155 Ocelic, N., Huber, A. \& Hillenbrand, R. Pseudoheterodyne detection for background-free near-field spectroscopy. Applied Physics Letters 89, 101124 (2006).

156 Xu, X. G., Gilburd, L. \& Walker, G. C. Phase stabilized homodyne of infrared scattering type scanning near-field optical microscopy. Applied Physics Letters 105, 263104 (2014).

157 Instruments, R. M. Material Data, <http://rmico.com/material-data > (2014).

158 Cvitkovic, A., Ocelic, N. \& Hillenbrand, R. Analytical model for quantitative prediction of material contrasts in scattering-type near-field optical microscopy. Optics express 15, 8550-8565 (2007).

159 Zeglinski, G., Niesterowicz, A. \& Gajda, J. in Laser Technology VIII: Applications of Lasers. 659810 (International Society for Optics and Photonics).

160 Carrasco, C., Ares, P., De Pablo, P. \& Gómez-Herrero, J. Cutting down the forest of peaks in acoustic dynamic atomic force microscopy in liquid. Review of Scientific Instruments 79, 126106 (2008).

161 Labuda, A. et al. Photothermal excitation for improved cantilever drive performance in tapping mode atomic force microscopy. Microscopy and Analysis28 3, S21-S25 (2014). 
162 Appavoo, K. et al. Ultrafast Phase Transition via Catastrophic Phonon Collapse Driven by Plasmonic Hot-Electron Injection. Nano Letters 14, 1127-1133, doi:10.1021/n14044828 (2014).

163 Kübler, C. et al. Coherent Structural Dynamics and Electronic Correlations during an Ultrafast Insulator-to-Metal Phase Transition in $\mathrm{VO}_{2}$. Physical Review Letters 99, 116401 (2007).

164 Morrison, V. R. et al. A photoinduced metal-like phase of monoclinic $\mathrm{VO}_{2}$ revealed by ultrafast electron diffraction. Science 346, 445-448, doi:10.1126/science.1253779 (2014).

$165 \mathrm{O} /$ 'Callahan, B. T. et al. Inhomogeneity of the ultrafast insulator-to-metal transition dynamics of $\mathrm{VO}_{2}$. Nat Commun 6, doi:10.1038/ncomms7849 (2015).

166 Tomczak, J. M. \& Biermann, S. Materials design using correlated oxides: Optical properties of vanadium dioxide. EPL (Europhysics Letters) 86, 37004 (2009).

167 Granqvist, C. G. Window coatings for the future. Thin Solid Films 193-194, Part 2, 730741, doi:http://dx.doi.org/10.1016/0040-6090(90)90225-3 (1990).

168 Babulanam, S. M., Eriksson, T. S., Niklasson, G. A. \& Granqvist, C. G. Thermochromic $\mathrm{VO}_{2}$ films for energy-efficient windows. Solar Energy Materials 16, 347-363, doi:http://dx.doi.org/10.1016/0165-1633(87)90029-3 (1987).

169 Jones, A. C., Berweger, S., Wei, J., Cobden, D. \& Raschke, M. B. Nano-optical Investigations of the Metal-Insulator Phase Behavior of Individual $\mathrm{VO}_{2}$ Microcrystals. Nano Letters 10, 1574-1581, doi:10.1021/n1903765h (2010).

170 Hryha, E., Rutqvist, E. \& Nyborg, L. Stoichiometric vanadium oxides studied by XPS. Surface and Interface Analysis 44, 1022-1025, doi:10.1002/sia.3844 (2012).

171 Zhu, Y. et al. Mesoscopic structural phase progression in photo-excited $\mathrm{VO}_{2}$ revealed by time-resolved x-ray diffraction microscopy. Scientific Reports 6, 21999, doi:10.1038/srep21999https://www.nature.com/articles/srep21999\#supplementaryinformation (2016).

172 Hongbo, Q. et al. The tetragonal-like to rutile structural phase transition in epitaxial $\mathrm{VO}_{2}$ $/ \mathrm{TiO}_{2}$ (001) thick films. New Journal of Physics 17, 113016 (2015).

173 Il'inskii, A. et al. Electron-beam modification of the parameters of the insulator-metal phase transition in vanadium dioxide films. Technical Physics Letters 39, 705-708 (2013).

174 Ko, Y., Ratner, B. \& Hoffman, A. Characterization of hydrophilic-hydrophobic polymeric surfaces by contact angle measurements. Journal of Colloid and Interface Science 82, 25-37 (1981).

175 Park, K., Lin, W. \& Paesani, F. Fast and Slow Proton Transfer in Ice: The Role of the Quasi-Liquid Layer and Hydrogen-Bond Network. The Journal of Physical Chemistry B 118, 8081-8089, doi:10.1021/jp501116d (2014).

176 Asay, D. B. \& Kim, S. H. Evolution of the Adsorbed Water Layer Structure on Silicon Oxide at Room Temperature. The Journal of Physical Chemistry B 109, 16760-16763, doi:10.1021/jp053042o (2005).

177 Merte, L. R. et al. Water-Mediated Proton Hopping on an Iron Oxide Surface. Science 336, 889-893, doi:10.1126/science.1219468 (2012).

178 Calleja, M., Tello, M. \& García, R. Size determination of field-induced water menisci in noncontact atomic force microscopy. Journal of applied physics 92, 5539-5542 (2002). 
179 Adhikari, S. et al. Charge Transfer to LaAlO3/SrTiO3 Interfaces Controlled by Surface Water Adsorption and Proton Hopping. Advanced Functional Materials 26, 5453-5459, doi:10.1002/adfm.201600820 (2016).

180 Gómez-Monivas, S., Sáenz, J. J., Calleja, M. \& García, R. Field-induced formation of nanometer-sized water bridges. Physical review letters 91, 056101 (2003).

$181 \mathrm{Xu}$, J., Kwak, K. J., Lee, J. L. \& Agarwal, G. Lifting and Sorting of Charged Au Nanoparticles by Electrostatic Forces in Atomic Force Microscopy. Small 6, 2105-2108, doi:10.1002/smll.201000924 (2010).

182 Li, Y., Maynor, B. W. \& Liu, J. Electrochemical AFM "Dip-Pen" Nanolithography. Journal of the American Chemical Society 123, 2105-2106, doi:10.1021/ja005654m (2001).

183 Liu, H., Hoeppener, S. \& Schubert, U. S. Nanoscale materials patterning by local electrochemical lithography. Advanced engineering materials 18, 890-902 (2016).

184 Ryu, Y. K. \& Garcia, R. Advanced oxidation scanning probe lithography. Nanotechnology 28, 142003 (2017).

185 Zhang, K. et al. Direct writing of electronic devices on graphene oxide by catalytic scanning probe lithography. Nature communications 3, 1194 (2012).

186 Lorenzoni, M. et al. Nanoscale reduction of graphene oxide thin films and its characterization. Nanotechnology 26, 285301 (2015).

187 Dagata, J. A. et al. Modification of hydrogen-passivated silicon by a scanning tunneling microscope operating in air. Applied Physics Letters 56, 2001-2003 (1990).

188 Sugimura, H. \& Nakagiri, N. Chemical approach to nanofabrication: modifications of silicon surfaces patterned by scanning probe anodization. Japanese journal of applied physics 34, 3406 (1995).

189 Vijaykumar, T., Raina, G., Heun, S. \& Kulkarni, G. Catalytic behavior of individual Au nanocrystals in the local anodic oxidation of Si surfaces. The Journal of Physical Chemistry C 112, 13311-13316 (2008).

$190 \mathrm{Wu}, \mathrm{C}$. et al. Hydrogen-Incorporation Stabilization of Metallic $\mathrm{VO}_{2}(\mathrm{R})$ Phase to Room Temperature, Displaying Promising Low-Temperature Thermoelectric Effect. Journal of the American Chemical Society 133, 13798-13801, doi:10.1021/ja203186f (2011).

191 Cazorla, C. Lattice Effects on the Formation of Oxygen Vacancies in Perovskite Thin Films. Physical Review Applied 7, 044025 (2017).

192 Petkov, V. et al. Structure of V2O5·nH2O Xerogel Solved by the Atomic Pair Distribution Function Technique. Journal of the American Chemical Society 124, 10157-10162, doi:10.1021/ja026143y (2002).

193 Wei, Q. et al. Hydrated vanadium pentoxide with superior sodium storage capacity. Journal of Materials Chemistry A 3, 8070-8075, doi:10.1039/C5TA00502G (2015).

194 Evans, H. T., Jnr \& Mrose, M. E. The crystal structures of three new vanadium oxide minerals. Acta Crystallographica 11，56-58, doi:doi:10.1107/S0365110X58000141 (1958).

195 Persson, K. Materials Data on VH2O3 (SG:15) by Materials Project. doi:10.17188/1206227 (2014).

196 Kats, M. A. et al. Vanadium Dioxide as a Natural Disordered Metamaterial: Perfect Thermal Emission and Large Broadband Negative Differential Thermal Emittance. Physical Review X 3, 041004 (2013). 
197 Kats, M. A. et al. Ultra-thin perfect absorber employing a tunable phase change material. Applied Physics Letters 101, 221101 (2012).

198 Zhang, Z. et al. Nanoscale Engineering in VO2 Nanowires via Direct Electron Writing Process. Nano letters 17, 851-855 (2017).

$199 \mathrm{Ni}, \mathrm{G}$. et al. Ultrafast optical switching of infrared plasmon polaritons in high-mobility graphene. Nature Photonics 10, 244-247 (2016).

200 Walla, F. et al. Anisotropic excitation of surface plasmon polaritons on a metal film by a scattering-type scanning near-field microscope with a non-rotationally-symmetric probe tip. Nanophotonics 7, 269-276 (2018).

201 Seidel, J. Propagation, Scattering and Amplification of Surface Plasmons in Thin Silver Films. (2005).

202 Maier, S. A. Plasmonics: fundamentals and applications. (Springer Science \& Business Media, 2007).

203 Burke, J., Stegeman, G. \& Tamir, T. Surface-polariton-like waves guided by thin, lossy metal films. Physical Review B 33, 5186 (1986).

204 Schubert, M., Tiwald, T. \& Herzinger, C. Infrared dielectric anisotropy and phonon modes of sapphire. Physical Review B 61, 8187 (2000).

205 Shtin, N. A., Romero, J. M. L. \& Prokhorov, E. Theory of fundamental microwave absorption in sapphire $\left(\alpha-\mathrm{Al}_{2} \mathrm{O}_{3}\right)$. 106, 104115, doi:10.1063/1.3259433 (2009).

206 Farmakidis, N. et al. Plasmonic nanogap enhanced phase-change devices with dual electrical-optical functionality. Science Advances 5, eaaw2687 (2019).

207 Alonso-González, P. et al. Resolving the electromagnetic mechanism of surface-enhanced light scattering at single hot spots. Nature communications 3, 684 (2012).

208 Olmon, R. L., Krenz, P. M., Jones, A. C., Boreman, G. D. \& Raschke, M. B. Near-field imaging of optical antenna modes in the mid-infrared. Optics express 16, 20295-20305 (2008).

209 Li, Y. et al. Patterned photonic crystals for hiding information. Journal of Materials Chemistry C 5, 4621-4628 (2017).

210 Folland, T. G. et al. Reconfigurable infrared hyperbolic metasurfaces using phase change materials. Nature communications 9, 1-7 (2018).

211 Genevet, P., Capasso, F., Aieta, F., Khorasaninejad, M. \& Devlin, R. Recent advances in planar optics: from plasmonic to dielectric metasurfaces. Optica 4, 139-152 (2017).

212 Lalanne, P. \& Hutley, M. Artificial media optical properties-subwavelength scale. Vol. 1 (Marcel Dekker, 2003).

213 Auton, J. Infrared transmission polarizers by photolithography. Applied optics 6, 10231027 (1967).

214 Choi, H., Ahn, J., Jung, J., Noh, T. \& Kim, D. Mid-infrared properties of a $\mathrm{VO}_{2}$ film near the metal-insulator transition. Physical Review B 54, 4621 (1996).

215 Luo, X. Subwavelength optical engineering with metasurface waves. Advanced Optical Materials 6, 1701201 (2018).

216 Hou, Y., Xiao, R., Tong, X., Dhuey, S. \& Yu, D. In Situ Visualization of Fast Surface Ion Diffusion in Vanadium Dioxide Nanowires. Nano letters 17, 7702-7709 (2017).

217 Lin, J. et al. Hydrogen diffusion and stabilization in single-crystal VO2 micro/nanobeams by direct atomic hydrogenation. Nano letters 14, 5445-5451 (2014). 
218 Gorai, P., Kondratenko, Y. V. \& Seebauer, E. G. Mechanism and kinetics of near-surface dopant pile-up during post-implant annealing. Journal of Applied Physics 111, 094510 (2012).

219 Schaub, R. et al. Oxygen-mediated diffusion of oxygen vacancies on the $\mathrm{TiO} 2$ (110) surface. Science 299, 377-379 (2003).

220 Bao, S. et al. Oxygen Vacancy Dynamics at Room Temperature in Oxide Heterostructures. ACS applied materials \& interfaces 10, 5107-5113 (2018).

221 Kahn, A. Fermi level, work function and vacuum level. Materials Horizons 3, 7-10 (2016).

222 Van Zeghbroeck, B. Principles of semiconductor devices. Colarado University 34 (2004).

223 Hynninen, T., AS, F. \& Barth, C. Polarized tips or surfaces: consequences in Kelvin probe force microscopy. e-Journal of Surface Science and Nanotechnology 9, 6-14 (2011).

224 Crank, J. The mathematics of diffusion. (Oxford university press, 1979).

225 Schnoor, J. L. Environmental modeling: fate and transport of pollutants in water, air, and soil. (John Wiley and Sons, 1996).

226 Brown, K. A., Satzinger, K. J. \& Westervelt, R. M. High spatial resolution Kelvin probe force microscopy with coaxial probes. Nanotechnology 23, 115703 (2012).

227 Soh, E. et al. Filamentary High-Resolution Electrical Probes for Nanoengineering. Nano Letters (2020).

228 Barker, A. S., Verleur, H. W. \& Guggenheim, H. J. Infrared Optical Properties of Vanadium Dioxide Above and Below the Transition Temperature. Physical Review Letters 17, 1286-1289 (1966).

229 Feinleib, J. \& Paul, W. Semiconductor-to-metal transition in V 2 O 3. Physical Review 155, 841 (1967).

230 Mitsuishi, T. On the phase transformation of VO2. Japanese Journal of Applied Physics 6 , 1060 (1967).

231 Verleur, H. W., Barker, A. S. \& Berglund, C. N. Optical Properties of $\mathrm{VO}_{2}$ between 0.25 and 5 eV. Physical Review 172, 788-798 (1968).

232 Dawson, P., Puygranier, B. \& Goudonnet, J. Surface plasmon polariton propagation length: A direct comparison using photon scanning tunneling microscopy and attenuated total reflection. Physical Review B 63, 205410 (2001).

233 Yang, Z. \& Ramanathan, S. Breakthroughs in photonics 2014: phase change materials for photonics. IEEE Photonics Journal 7, 1-5 (2015).

234 contributors, W. Photonic integrated circuit, <https://en.wikipedia.org/w/index.php?title=Photonic_integrated_circuit\&oldid=9437278 $\underline{90}>$

235 contributors, W. Optical transistor, <https://en.wikipedia.org/w/index.php?title=Optical_transistor\&oldid=947677147>

236 Stegmaier, M. et al. Aluminum nitride nanophotonic circuits operating at ultraviolet wavelengths. Applied Physics Letters 104, 091108 (2014).

237 Pernice, W. H. \& Bhaskaran, H. Photonic non-volatile memories using phase change materials. Applied Physics Letters 101, 171101 (2012).

238 Rios, C., Hosseini, P., Wright, C. D., Bhaskaran, H. \& Pernice, W. H. On-chip photonic memory elements employing phase-change materials. Advanced Materials 26, 1372-1377 (2014). 
239 Ríos, C. et al. Integrated all-photonic non-volatile multi-level memory. Nature Photonics 9, 725 (2015).

240 Bertels, K., Cardoso, J. M. \& Vassiliadis, S. Reconfigurable Computing: Architectures and Applications: Second International Workshop, ARC 2006, Delft, The Netherlands, March 1-3, 2006 Revised Selected Papers. Vol. 3985 (Springer, 2006).

241 Hao, Q. et al. VO2/TiN Plasmonic Thermochromic Smart Coatings for Room-Temperature Applications. Advanced materials 30, 1705421 (2018).

242 Kruger, B. A., Joushaghani, A. \& Poon, J. K. Design of electrically driven hybrid vanadium dioxide (VO 2) plasmonic switches. Optics Express 20, 23598-23609 (2012).

243 Cao, T., Simpson, R. E. \& Cryan, M. J. Study of tunable negative index metamaterials based on phase-change materials. JOSA B 30, 439-444 (2013).

244 Tittl, A. et al. A switchable mid-infrared plasmonic perfect absorber with multispectral thermal imaging capability. Advanced Materials 27, 4597-4603 (2015).

245 Chen, Y. et al. Engineering the phase front of light with phase-change material based planar lenses. Scientific reports 5, 1-7 (2015).

246 Kargar, R., Rouhi, K. \& Abdolali, A. Reprogrammable multifocal THz metalens based on metal-insulator transition of VO2-assisted digital metasurface. Optics Communications 462, 125331 (2020).

247 Cohen, R. E. Origin of ferroelectricity in perovskite oxides. Nature 358, 136 (1992).

248 Hill, N. A. (ACS Publications, 2000).

249 Scott, J. \& Blinc, R. Multiferroic magnetoelectric fluorides: why are there so many magnetic ferroelectrics? Journal of Physics: Condensed Matter 23, 113202 (2011).

250 Ederer, C. \& Spaldin, N. A. Electric-field-switchable magnets: the case of BaNiF 4. Physical Review B 74, 020401 (2006).

251 Ederer, C. \& Spaldin, N. A. Origin of ferroelectricity in the multiferroic barium fluorides Ba M F 4: A first principles study. Physical Review B 74, 024102 (2006).

252 DiDomenico Jr, M., Eibschütz, M., Guggenheim, H. \& Camlibel, I. Dielectric behavior of ferroelectric BaMF4 above room temperature. Solid State Communications 7, 1119-1122 (1969).

253 Keve, E., Abrahams, S. \& Bernstein, J. Ferroelectric paraelastic paramagnetic barium cobalt fluoride, BaCoF4, crystal structure. The Journal of Chemical Physics 53, 3279-3287 (1970).

254 Cox, D., Eibschütz, M., Guggenheim, H. \& Holmes, L. Neutron Diffraction Study of the Magnetic Structure of BaNiF4. Journal of Applied Physics 41, 943-945 (1970).

255 Eibschütz, M. \& Guggenheim, H. Antiferromagnetic-piezoelectric crystals: Bame4 (m= mn, fe, co and ni). Solid State Communications 6, 737-739 (1968).

256 Eibschütz, M., Guggenheim, H., Wemple, S., Camlibel, I. \& DiDomenico Jr, M. Ferroelectricity in BaM2+ F4. Physics Letters A 29, 409-410 (1969).

257 Eibschütz, M., Holmes, L., Guggenheim, H. \& Cox, D. Magnetic Structure of the TwoDimensional Antiferromagnet BaCo F 4. Physical Review B 6, 2677 (1972).

258 Glass, A. \& FSL, H. OBSERVATION OF ANOMALOUS PYROELECTRIC BEHAVIOUR IN BANIF4 DUE TO A COOPERATIVE MAGNETIC SINGULARITY. (1977).

259 Scott, J. Mechanisms of dielectric anomalies in BaMn F 4. Physical Review B 16, 2329 (1977). 
260 Scott, J. Phase transitions in BaMnF4. Reports on Progress in Physics 42, 1055 (1979).

261 Kleemann, W., Schafer, F. \& Nouet, J. Linear magnetic birefringence and double-excitonic transitions of the two-dimensional antiferromagnet BaNiF4. Journal of Physics C: Solid State Physics 14, 4447 (1981).

262 HG, V. \& P, B. Vol. 55 342-\& (SPRINGER VERLAG 175 FIFTH AVE, NEW YORK, NY 10010, 1968).

263 Borisov, P. et al. Multiferroic BaCoF4 in thin film form: ferroelectricity, magnetic ordering, and strain. ACS applied materials \& interfaces 8, 2694-2703 (2016).

264 Víllora, E. G. et al. Ferroelectric and optical properties of single crystal Ba Zn F 4. Applied physics letters 90, 192909 (2007).

265 Dawber, M., Rabe, K. \& Scott, J. Physics of thin-film ferroelectric oxides. Reviews of modern physics 77, 1083 (2005).

266 Güthner, P. \& Dransfeld, K. Local poling of ferroelectric polymers by scanning force microscopy. Applied Physics Letters 61, 1137-1139 (1992).

267 Kalinin, S. V. et al. Vector piezoresponse force microscopy. Microscopy and Microanalysis 12, 206-220 (2006).

$268 \mathrm{Xu}, \mathrm{R}$. et al. Ferroelectric polarization reversal via successive ferroelastic transitions. Nature materials 14, 79 (2015).

269 Balke, N. et al. Deterministic control of ferroelastic switching in multiferroic materials. Nature nanotechnology 4, 868 (2009).

270 Zhao, T. et al. Electrical control of antiferromagnetic domains in multiferroic BiFeO 3 films at room temperature. Nature materials 5, 823 (2006).

271 Dai, W. et al. Large and reconfigurable infrared photothermoelectric effect at oxide interfaces. Nano letters 19, 7149-7154 (2019).

272 Clavero, C. Plasmon-induced hot-electron generation at nanoparticle/metal-oxide interfaces for photovoltaic and photocatalytic devices. Nat Photon 8, 95-103, doi:10.1038/nphoton.2013.238 (2014).

273 Zolotavin, P., Evans, C. \& Natelson, D. Photothermoelectric Effects and Large Photovoltages in Plasmonic Au Nanowires with Nanogaps. The Journal of Physical Chemistry Letters 8, 1739-1744, doi:10.1021/acs.jpclett.7b00507 (2017).

274 Li, W. \& Valentine Jason, G. in Nanophotonics Vol. 6177 (2017).

275 Zheng, B. Y. et al. Distinguishing between plasmon-induced and photoexcited carriers in a device geometry. $\quad 6, \quad 7797, \quad$ doi:10.1038/ncomms8797 https://www.nature.com/articles/ncomms8797\#supplementary-information (2015).

276 Fang, J. et al. Enhanced Graphene Photodetector with Fractal Metasurface. Nano Letters 17, 57-62, doi:10.1021/acs.nanolett.6b03202 (2017).

277 Cen, C. et al. Nanoscale control of an interfacial metal-insulator transition at room temperature. Nat Mater 7, 298-302, doi: http://www.nature.com/nmat/journal/v7/n4/suppinfo/nmat2136_S1.html (2008).

278 Dai, W. et al. Tailoring LaAlO3/SrTiO3 Interface Metallicity by Oxygen Surface Adsorbates. Nano Letters 16, 2739-2743, doi:10.1021/acs.nanolett.6b00421 (2016).

279 Dai, W. et al. Tailoring the Doping Mechanisms at Oxide Interfaces in Nanoscale. Nano Letters 17, 5620-5625, doi:10.1021/acs.nanolett.7b02508 (2017).

280 Cen, C. et al. Nanoscale control of an interfacial metal-insulator transition at room temperature. Nature materials 7, 298-302 (2008). 
281 Landsberg, P. T. \& Klimpke, C. Theory of the Schottky barrier solar cell. Proceedings of the Royal Society of London. A. Mathematical and Physical Sciences 354, 101-118, doi:10.1098/rspa.1977.0058 (1977).

282 Srivastava, S., Swami, N. K. \& Srivastava, G. P. Efficiency of Schottky barrier solar cells. physica status solidi (a) 58, 343-348, doi:10.1002/pssa.2210580203 (1980).

283 Meevasana, W. et al. Creation and control of a two-dimensional electron liquid at the bare SrTiO3 surface. Nat Mater 10, 114-118, doi:10.1038/nmat2943 (2011).

284 Broido, D. A. \& Reinecke, T. L. Effect of superlattice structure on the thermoelectric figure of merit. Physical Review B 51, 13797-13800 (1995).

285 Hicks, L. D. \& Dresselhaus, M. S. Effect of quantum-well structures on the thermoelectric figure of merit. Physical Review B 47, 12727-12731 (1993).

286 Pallecchi, I. et al. Seebeck effect in the conducting $\mathrm{LaAlO}_{3} / \mathrm{SrTiO}_{3}$ interface. Physical Review B 81, 085414 (2010).

287 Ohta, H. et al. Giant thermoelectric Seebeck coefficient of a two-dimensional electron gas in SrTiO3. Nat Mater 6, 129-134 (2007).

288 Mashhadi, S., Duong, D. L., Burghard, M. \& Kern, K. Efficient Photothermoelectric Conversion in Lateral Topological Insulator Heterojunctions. Nano Letters 17, 214-219, doi:10.1021/acs.nanolett.6b03851 (2017).

289 Basko, D. A Photothermoelectric Effect in Graphene. Science 334, 610-611, doi:10.1126/science.1214560 (2011).

290 Johnson, P. B. \& Christy, R. W. Optical Constants of the Noble Metals. Physical Review B 6, 4370-4379 (1972). 